Problemas de valores de contorno envolvendo o operador biharmônico

Vanderley Alves Ferreira Junior 

SERVIÇO DE PÓS-GRADUAÇÃO DO ICMC-USP

Data de Depósito: / /

Assinatura:

\title{
Problemas de valores de contorno envolvendo o operador biharmônico
}

\author{
Vanderley Alves Ferreira Junior
}

Orientador: Prof. Dr. Ederson Moreira dos Santos

Dissertação apresentada ao Instituto de Ciências Matemáticas e de Computação - ICMC-USP, como parte dos requisitos para obtenção do título de Mestre em Ciências - Matemática . VERSÃO REVISADA 
Ficha catalográfica elaborada pela Biblioteca Prof. Achille Bassi e Seção Técnica de Informática, ICMC/USP, com os dados fornecidos pelo(a) autor(a)

\begin{tabular}{|c|c|}
\hline \multirow[t]{3}{*}{ F383p } & $\begin{array}{l}\text { Ferreira Junior, Vanderley Alves } \\
\quad \text { Problemas de valores de contorno envolvendo o } \\
\text { operador biharmônico / Vanderley Alves Ferreira } \\
\text { Junior; orientador Ederson Moreira dos Santos. -- } \\
\text { São Carlos, } 2013 \text {. } \\
\quad 92 \text { p. }\end{array}$ \\
\hline & $\begin{array}{l}\text { Dissertação (Mestrado - Programa de Pós-Graduação en } \\
\text { Matemática)-- Instituto de Ciências Matemáticas e } \\
\text { de Computação, Universidade de São Paulo, } 2013 .\end{array}$ \\
\hline & $\begin{array}{l}\text { 1. Operador biharmônico. 2. Condições de contorno } \\
\text { de Dirichlet, Navier e Steklov. 3. Função de Green. } \\
\text { 4. Preservação de positividade. 5. Problemas } \\
\text { semilineares. I. Moreira dos Santos, Ederson, } \\
\text { orient. II. Título. }\end{array}$ \\
\hline
\end{tabular}


A minha Marine. 


\section{Agradecimentos}

Agradeço a Deus por ter me sustentado e guiado durante a realização deste trabalho e pelas pessoas incríveis que Ele pôs em meu caminho.

A minha querida esposa Marine, sem você eu nunca teria terminado. Obrigado por acreditar em mim e por lutar ao meu lado todos os dias.

Aos meus pais Vanderley e Luci e aos meu sogros Valdir e Elenice, que apesar da distância sempre se fizeram presentes com seu apoio, carinho e orações.

Ao meu orientador, professor Ederson, por ter me ajudado sempre que precisei ao longo destes dois anos.

Aos demais professores com quem tanto tenho aprendido, em especial ao professor Fredy Suárez.

À CAPES pelo apoio financeiro. 
Com abelhas ou sem abelhas, os problemas interessantes da Matemática têm, para o pesquisador, a doçura do mel.

Ary Quintela. 


\section{Resumo}

Estudamos o problema de valores de contorno

$$
\left\{\begin{array}{c}
\Delta^{2} u=f \text { em } \Omega, \\
\mathcal{B} u=0 \text { em } \partial \Omega,
\end{array}\right.
$$

em um aberto limitado $\Omega \subset \mathbb{R}^{N}$, sob diferentes condições de contorno. As questões de existência e positividade de soluções para este problema são abordadas com condições de contorno de Dirichlet, Navier e Steklov. Deduzimos condições de contorno naturais através do estudo de um modelo para uma placa com carga estática.

Estudamos ainda propriedades do primeiro autovalor de $\Delta^{2}$ e o problema semilinear

$$
\left\{\begin{aligned}
\Delta^{2} u & =F(u) & \operatorname{em} \Omega \\
u & =\frac{\partial u}{\partial \nu}=0 & \text { em } \partial \Omega
\end{aligned}\right.
$$

para não-linearidades do tipo $F(t)=|t|^{p-1} t, p \neq 1, p>0$. Para tal problema estudamos existência e não-existência de soluções e positividade. 


\section{Abstract}

We study the boundary value problem

$$
\left\{\begin{array}{rll}
\Delta^{2} u=f & \text { in } \Omega, \\
\mathcal{B} u=0 & \text { in } \partial \Omega,
\end{array}\right.
$$

in a bounded open $\Omega \subset \mathbb{R}^{N}$ under different boundary conditions. The questions of existence and positivity of solutions for this problem are addressed with Dirichlet, Navier and Steklov boundary conditions. We deduce natural boundary conditions through the study of a model for a plate with static load.

We also study properties of the first eigenvalue of $\Delta^{2}$ and the semi-linear problem

$$
\left\{\begin{aligned}
\Delta^{2} u & =F(u) & & \text { in } \Omega \\
u & =\frac{\partial u}{\partial \nu}=0 & & \text { in } \partial \Omega
\end{aligned}\right.
$$

for non-linearities like $F(t)=|t|^{p-1} t, p \neq 1, p>0$. For such problem we study existence and non-existence of solutions and its positivity. 


\section{Sumário}

Introdução 11

Notações

1 Modelos de ordem quatro 5

1.1 Caso unidimensional . . . . . . . . . . . . . . . . . 5

1.2 O Laplaciano de uma função sobre uma curva de nível . . . . . . . . . . . 7

1.3 Caso bidimensional . . . . . . . . . . . . . . . . . . . . 12

2 Problemas lineares $\quad 15$

2.1 A condição de Dirichlet . . . . . . . . . . . . . . . . . . . . . 19

2.2 A função de Green . . . . . . . . . . . . . . . . . . . . . . . . 21

2.2.1 A função de Green da bola unitária $B$. . . . . . . . . . . . . . 22

2.2.2 A função de Green dos limaçons de Pascal . . . . . . . . . . . . . . 23

2.3 A condição de Navier . . . . . . . . . . . . . . . . . . . . . . 26

2.4 A condição de Steklov . . . . . . . . . . . . . . . . . . . 28

2.5 Problemas de autovalor . . . . . . . . . . . . . . . 36

3 Uma classe de problemas semilineares $\quad 47$

3.1 Problema subcrítico . . . . . . . . . . . . . . . . . . . . 48

3.1 .1 O caso sublinear . . . . . . . . . . . . . . . . . 49

3.1 .2 O caso superlinear . . . . . . . . . . . . . . . 51

3.1 .3 Solução de energia mínima . . . . . . . . . . . . . . . 55

3.2 Resultados de não-existência . . . . . . . . . . . . . . . . . 61

$\begin{array}{ll}\text { A Espaços de funções } & 69\end{array}$

$\begin{array}{ll}\text { B Cálculo em espaços de Banach } & 75\end{array}$

B.1 Funcionais diferenciáveis . . . . . . . . . . . . . . . . 75 
B.2 Exemplos . . . . . . . . . . . . . . . . . . . . . 79

C Pontos críticos de um funcional 


\section{Introdução}

O operador biharmônico é definido por $\Delta^{2}=\Delta(\Delta)$, onde $\Delta$ denota o operador Laplaciano.

A equação $\Delta^{2} u=f$, conhecida como equação da placa, precisa ser complementada com condições de contorno apropriadas para que se obtenha problemas matematicamente bem postos e com significado físico. Nesta dissertação estão apresentados resultados sobre a existência e positividade de soluções de problemas de valores de contorno do tipo

$$
\left\{\begin{aligned}
\Delta^{2} u & =f \text { em } \Omega, \\
\mathcal{B} u & =0 \text { em } \partial \Omega,
\end{aligned}\right.
$$

além de problemas semilineares como

$$
\left\{\begin{array}{rrrr}
\Delta^{2} u & = & F(u) & \text { em } \Omega \\
\mathcal{B} u & = & 0 & \text { em } \partial \Omega
\end{array}\right.
$$

para algumas classes de não-linearidades $F$ e para diferentes condições de contorno $\mathcal{B}$.

Muitas técnicas para problemas envolvendo operadores elípticos de ordem dois não podem ser aplicadas a problemas de ordem superior devido à falta de um princípio do máximo, e porque para $u \in H^{2}(\Omega)$, em geral $|u| \notin H^{2}(\Omega)$. Estudamos situações em que o biharmônico possui a propriedade de preservação de positividade e quais propriedades de problemas de ordem dois são mantidas.

Por se tratar de uma equação de ordem 4, há diversas escolhas possíveis de conjuntos de condições de contorno apropriadas do ponto de vista matemático. Para mais detalhes veja [3].

Em analogia ao caso do operador $\Delta$ em que as condições mais estudadas são as de Dirichlet, Neumann e Robin, que possuem significado físico, para o operador biharmônico estudamos as condições de Dirichlet, Navier e Steklov. Cada uma possui particularidades 
que serão comparadas nos Capítulos 2 e 3.

No Capítulo 1 estudamos dois modelos da teoria da elasticidade para derivar condições de contorno naturais para os problemas de contorno envolvendo o biharmônico.

O segundo capítulo trata do problema linear sob as condições de contorno de Dirichlet, Navier e Steklov, abordando existência de soluções e preservação de positividade. Veremos por exemplo que a equação permite, sob condições de Navier e Steklov, uma decomposição em um sistema de equações de ordem dois. Sob estas condições o biharmônico preserva positividade. Por outro lado, sob condição de Dirichlet esta propriedade é perdida em alguns domínios. Estudamos ainda as propriedades do primeiro autovalor do biharmônico sob cada condição de contorno e sua relação com a preservação de positividade.

No Capítulo 3 estudamos existência e não-existência de soluções para uma classe de problemas semilineares, com não-linearidade do tipo

$$
F(u)=|u|^{p-1} u
$$

bem como propriedades de soluções particulares, como soluções de energia mínima. 


\section{Notações}

1. $N$ dimensão do espaço.

2. $C, c$ constantes positivas.

3. $\Omega$ denota um aberto não-vazio contido em $\mathbb{R}^{N}$.

4. $\partial \Omega$ denota a fronteira de $\Omega, \partial \Omega=\bar{\Omega} \backslash \Omega$.

5. $\bar{A}$ é o fecho do conjunto $A \subset X$ na topologia de $X$.

6. $B \subset \mathbb{R}^{N}$ é a bola unitária aberta centrada em 0 , isto é, $B=B_{1}(0)$.

7. $B_{r}(x) \subset \mathbb{R}^{N}$ é a bola aberta de raio $r>0$ e centro $x ; B_{r}(x)=\left\{y \in \mathbb{R}^{N} ;\|x-y\|<r\right\}$.

8. $\bar{B}_{r}(x)$ é a bola fechada de raio $r>0$ e centro $x$.

9. $S_{r}^{N}$ é o conjunto de todos os $y \in \mathbb{R}^{N}$ tais que $\|y\|=r$.

10. $\nu$ denota o vetor normal exterior à fronteira de $\Omega$.

11. $u_{i}=\frac{\partial u}{\partial x_{i}}$ denota a derivada parcial de $u$ em relação à i-ésima coordenada.

12. $D u$ denota o gradiente de $u$.

13. $\Delta u=\sum_{i=1}^{N} u_{i i}$ é o Laplaciano de $u$.

14. $\Delta^{2} u=\Delta(\Delta u)=-\Delta(-\Delta u)=\sum_{i=1}^{N} \sum_{j=1}^{N} u_{i i j j}$ é o biharmônico de $u$.

15. $\frac{\partial u}{\partial \nu}$ é a derivada de $u$ na direção do vetor normal à fronteira $\nu$.

16. $\int_{\Omega} F d x$ denota a integral de Lebesgue da função $F$ no conjunto $\Omega$. 
17. $\int_{\partial \Omega} F d S$ denota a integral de Lebesgue de $F: \bar{\Omega} \longrightarrow \mathbb{R}$ na medida $(N-1)$
dimensional de $\partial \Omega$.

18. $\mathcal{C}^{0}(\bar{\Omega})$ é o espaço das funções contínuas em $\bar{\Omega}$.

19. $\mathcal{C}^{k}(\Omega)$, para $k \in \mathbb{N}$ é o espaço das funções $u: \Omega \longrightarrow \mathbb{R}$ com derivadas de ordem $k$ contínuas em $\Omega$.

20. $\mathcal{C}^{\infty}(\Omega)=\bigcap_{k \geq 1} \mathcal{C}^{k}(\Omega)$

21. $\mathcal{C}_{c}^{\infty}(\Omega)$ é o espaço das funções $u \in \mathcal{C}^{\infty}(\Omega)$ com suporte compacto contido em $\Omega$.

22. $\mathcal{C}_{0}^{0}(\bar{\Omega})$ é o espaço das funções $u \in \mathcal{C}^{0}(\bar{\Omega})$ que se anulam na fronteira de $\Omega$.

23. $H^{1}(\Omega)$ é o espaço de Sobolev de funções $u \in L^{2}(\Omega)$ que possuem derivadas fracas em $L^{2}(\Omega)$; ver Apêndice A.

24. $H^{2}(\Omega)$ é o espaço de Sobolev de funções $u \in H^{1}(\Omega)$ que possuem derivadas fracas em $H^{1}(\Omega)$.

25. $H_{0}^{j}(\Omega), j \in\{1,2\}$ é o fecho de $\mathcal{C}_{c}^{\infty}(\Omega)$ em $\mathcal{H}^{j}$; ver Apêndice A.

26. $T(u)$ é o traço de $u \in H^{1}(\Omega)$; ver Teorema A.7.

27. $X$ denota um espaço de Banach, $\mathcal{H}$ denota um espaço de Hilbert.

28. $X^{*}$ é o espaço dual do espaço de Banach $X$.

29. $|t|=\max \{t,-t\}$ é o módulo de $t \in \mathbb{R}$.

30. $t^{+}=\max \{t, 0\}, t^{-}=\max \{-t, 0\}, t \in \mathbb{R}$.

31. $\delta_{i j}$ é o operador $\delta$ de Dirac, $\delta_{i j}=1$ se $i=j$ e $\delta_{i j}=0$ se $i \neq j$.

32. $\|$ \| denota a norma no espaço de Banach $X$.
(a) $\|x\|=\left(\sum_{i=1}^{N} x_{i}^{2}\right)^{\frac{1}{2}}$, se $x=\left(x_{i}\right) \in \mathbb{R}^{N}$.
(b) $\|u\|=\left(\int_{\Omega}(\Delta u)^{2} d x\right)^{\frac{1}{2}}$, se $u \in H_{0}^{2}(\Omega)$ ou $u \in H^{2}(\Omega) \cap H_{0}^{1}(\Omega)$.
(c) $\|u\|_{p}=\left(\int_{\Omega}|u|^{p} d x\right)^{\frac{1}{p}}$, se $u \in L^{p}(\Omega), 1 \leq p<\infty$.

33. $G_{\Omega}$ a função de Green de $\Delta^{2}$ em $\Omega$ sob condição de Dirichlet. 


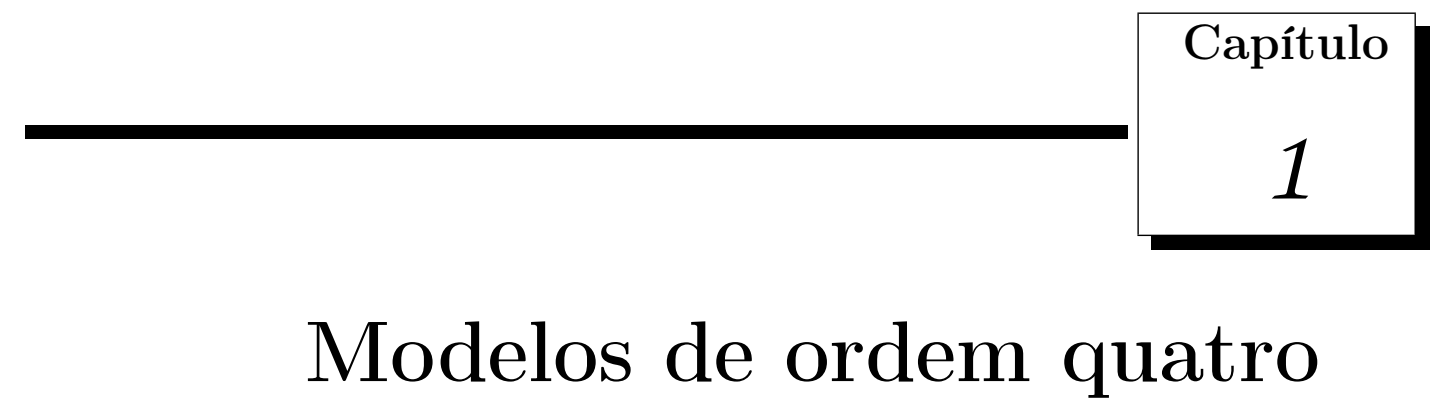

Neste capítulo estudamos dois modelos que levam a problemas de valores de contorno de ordem quatro, um para uma viga e o análogo para uma placa bidimensional. A partir da análise dos funcionais de energia chegamos às condições de contorno naturais para o operador biharmônico.

\subsection{Caso unidimensional}

Considere uma viga ideal unidimensional de comprimento $L$, inicialmente posicionada horizontalmente, sobre a qual é posta uma certa carga de peso atuando na vertical. Esta viga pode ser representada pelo gráfico de uma função $u:[0, L] \longrightarrow \mathbb{R}$, de modo que $u(t)$ representa a variação da posição do ponto correspondente da viga em relação ao equilíbrio.

A energia elástica acumulada na viga deve-se à resistência ao aumento de comprimento e à resistência à flexão, isto é, envergar-se. Um modelo para a energia elástica desta viga é

$$
J_{E}(u)=\int_{0}^{L}\left(\sqrt{1+u^{\prime}(x)^{2}}-1+\frac{u^{\prime \prime}(x)^{2}}{\left(1+u^{\prime}(x)^{2}\right)^{3}} \sqrt{1+u^{\prime}(x)^{2}}\right) d x,
$$

onde o primeiro termo representa a variação de comprimento de arco e o segundo representa a curvatura.

Se os extremos da viga puderem se mover livremente na direção horizontal, então não ocorre aumento de comprimento e o primeiro termo pode ser ignorado. Neste caso, 
denotando $p:[0, L] \longrightarrow \mathbb{R}$ a carga sobre a viga, a energia mecânica da viga seria

$$
J(u)=\int_{0}^{L}\left(\frac{u^{\prime \prime}(x)^{2}}{\left(1+u^{\prime}(x)^{2}\right)^{3}} \sqrt{1+u^{\prime}(x)^{2}}-p(x) u(x)\right) d x
$$

Uma função $u \in H^{2}([0, L]) \cap H_{0}^{1}([0, L])$ minimizando $J$ seria uma aproximação para a posição de equilíbrio da viga, sob estas hipóteses. Observe que se a curva é parametrizada por $\gamma(x)=(x, u(x))$, então $\frac{u^{\prime \prime}(x)^{2}}{\left(1+u^{\prime}(x)^{2}\right)^{3}}$ é a curvatura no ponto $\gamma(x)$. Para deformações pequenas, isto é, assumindo $u^{\prime} \approx 0$, podemos aproximar a energia da viga por

$$
J(u)=\int_{0}^{L}\left(\frac{1}{2} u^{\prime \prime}(x)^{2}-p(x) u(x)\right) d x
$$

Suponha que $u \in \mathcal{C}^{4}([0, L])$. Então para toda função $v \in \mathcal{C}^{2}([0, L])$,

$$
\begin{aligned}
J^{\prime}(u) v & =\int_{0}^{L}\left(u^{\prime \prime}(x) v^{\prime \prime}(x)-p(x) v(x)\right) d x \\
& =\left.u^{\prime \prime}(x) v^{\prime}(x)\right|_{0} ^{L}-\int_{0}^{L}\left(u^{\prime \prime \prime}(x) v^{\prime}(x)+p(x) v(x)\right) d x \\
& =\left.u^{\prime \prime}(x) v^{\prime}(x)\right|_{0} ^{L}-\left.u^{\prime \prime \prime}(x) v(x)\right|_{0} ^{L}+\int_{0}^{L}\left(u^{(4)}(x)-p(x)\right) v(x) d x
\end{aligned}
$$

Assim, com certas condições de fronteira impostas, os termos de fronteira se anulam e obtemos a equação de Euler-Lagrange para este funcional,

$$
u^{(4)}=p(x)
$$

Assim, as condições de fronteira apropriadas são:

$$
\begin{gathered}
\left\{\begin{array}{l}
u(0)=u(L)=0, \\
u^{\prime}(0)=u^{\prime}(L)=0,
\end{array}\right. \\
\left\{\begin{array}{l}
u(0)=u(L)=0, \\
u^{\prime \prime}(0)=u^{\prime \prime}(L)=0,
\end{array}\right. \\
\left\{\begin{array}{l}
u^{\prime}(0)=u^{\prime}(L)=0, \\
u^{\prime \prime \prime}(0)=u^{\prime \prime \prime}(L)=0,
\end{array}\right. \\
\left\{\begin{array}{l}
u^{\prime \prime}(0)=u^{\prime \prime}(L)=0, \\
u^{\prime \prime \prime}(0)=u^{\prime \prime \prime}(L)=0 .
\end{array}\right.
\end{gathered}
$$


A condição de contorno correspondente à viga engastada é (1.5), conhecida como condição de Dirichlet. Para a viga articulada, temos a condição de Navier (1.6). A condição (1.8) representa uma viga livre para se mover verticalmente nas extremidades, e a condição (1.7) um mecanismo que permite movimento vertical, mas impede que ela incline-se nas extremidades. Para mais detalhes veja [12, p. 3].

As condições podem aparecer combinadas. Por exemplo, um modelo para uma viga que está engastada em uma extremidade e livre na outra seria

$$
\left\{\begin{array}{cccc}
u^{(4)}= & p(x) & \mathrm{em}(0, L), \\
u(0)=u^{\prime}(0) & = & 0 \\
u^{\prime \prime}(L)= & u^{\prime \prime \prime}(L)= & 0 .
\end{array}\right.
$$

Esta viga é conhecida como viga em balanço ou cantilever.

\subsection{O Laplaciano de uma função sobre uma curva de nível}

Sejam $\Omega \subset \mathbb{R}^{2}$ um aberto, $u \in \mathcal{C}^{2}(\bar{\Omega})$. Suponha que $p=\left(x_{0}, y_{0}\right) \in u^{-1}(0)$ e que $D u(p) \neq 0$. Nesta seção mostramos que

$$
\Delta u(p)=\frac{\partial^{2} u}{\partial \nu^{2}}(p)+k(p) \frac{\partial u}{\partial \nu}(p)
$$

onde $k$ é a curvatura da curva de nível $u^{-1}(0)$ e $\nu$ é o vetor normal a esta.

A condição $D u(p) \neq 0$ nos garante que em uma vizinhança de $p, u^{-1}(0)$ é de fato uma curva.

Proposição 1.1. Se $\Omega \subset \mathbb{R}^{2}$ é um aberto, $p \in \Omega$, u é uma função de classe $\mathcal{C}^{1}(\Omega)$, $u(p)=0$ e $D u(p) \neq 0$, então existem um aberto $A \subset \Omega$, um intervalo $I \subset \mathbb{R}$ e uma aplicação $\gamma: I \longrightarrow \Omega$ de classe $\mathcal{C}^{1}$, tal que $\gamma(I)=A \cap u^{-1}(0)$.

Demonstração. Esta proposição é um caso particular do teorema função implícita, veja por exemplo [16, p. 295].

Teorema 1.2. Se $\Omega \subset \mathbb{R}^{2}$ é um aberto, $u \in \mathcal{C}^{2}(\bar{\Omega}), p=\left(x_{0}, y_{0}\right) \in u^{-1}(0)$ e $D u(p) \neq 0$, então

$$
\Delta u(p)=\frac{\partial^{2} u}{\partial \nu^{2}}(p)+k(p) \frac{\partial u}{\partial \nu}(p)
$$


Demonstração. Pela Proposição 1.1 acima, existem $I \subset \mathbb{R}$ aberto e $\gamma: I \longrightarrow \Omega$, de classe $\mathcal{C}^{1}$, uma parametrização de $u^{-1}(0)$ em uma vizinhança de $p$. Considere a função $\psi: J \longrightarrow \Omega$, onde $J \subset I, 0 \in J$, com coordenadas $\psi=(f, g)$, as quais são as soluções de

$$
\left\{\begin{aligned}
f^{\prime}(t) & =-u_{y}(f(t), g(t)), & & \operatorname{com} f(0)=x_{0} \\
g^{\prime}(t) & =u_{x}(f(t), g(t)), & & \operatorname{com} g(0)=y_{0}
\end{aligned}\right.
$$

Tal solução existe e é de classe $\mathcal{C}^{1}$; veja [11, Teorema 1.9].

Agora, definindo $m=u \circ \psi$ e tomando $s \in J$, podemos calcular

$$
\begin{aligned}
m^{\prime}(s) & =u_{x}(\psi(s)) f^{\prime}(s)+u_{y}(\psi(s)) g^{\prime}(s) \\
& =u_{x}(\psi(s))\left(-u_{y}(\psi(s))\right)+u_{y}(\psi(s)) u_{x}(\psi(s)) \\
& =0
\end{aligned}
$$

Logo $m$ é constante, ou seja, $u$ é constante sobre a imagem de $\psi$. Como $u(\psi(0))=u(p)=$ 0 , segue que $u^{-1}(0) \supset \psi(J)$. Concluímos então que $\psi$ é uma parametrização para a curva $u^{-1}(0)$ em uma vizinhança de $p$.

Usando a parametrização $\psi$, podemos calcular a curvatura em função de $u$ e de suas derivadas, usando a expressão da curvatura de [8, p. 25]:

$$
\begin{aligned}
k(s)= & \frac{\operatorname{det}\left(\psi^{\prime}(s), \psi^{\prime \prime}(s)\right)}{\left\|\psi^{\prime}(s)\right\|^{3}} \\
= & \frac{f^{\prime}(s) g^{\prime \prime}(s)-f^{\prime \prime}(s) g^{\prime}(s)}{\left(f^{\prime 2}(s)+g^{\prime 2}(s)\right)^{\frac{3}{2}}} \\
= & \frac{-u_{y}(\psi(s))\left(u_{x x}(\psi(s))\left(-u_{y}(\psi(s))\right)+\left(u_{x y}(\psi(s))\right)\left(u_{x}(\psi(s))\right)\right.}{\left(u_{x}^{2}(\psi(s))+\left(-u_{y}\right)^{2}(\psi(s))\right)^{\frac{3}{2}}}+ \\
& -\frac{u_{x}(\psi(s))\left(-u_{y y}(\psi(s)) u_{x}(\psi(s))+u_{y}(\psi(s)) u_{x y}(\psi(s))\right)}{\left(u_{x}^{2}(\psi(s))+\left(-u_{y}\right)^{2}(\psi(s))\right)^{\frac{3}{2}}} \\
= & \frac{\left(u_{y}^{2}(\psi(s)) u_{x x}(\psi(s))-2 u_{x}(\psi(s)) u_{y}(\psi(s)) u_{x y}(\psi(s))+u_{x}^{2}(\psi(s)) u_{y y}(\psi(s))\right)}{\|D u(\psi(s))\|^{3}} .
\end{aligned}
$$

O vetor normal à curva $\nu(s)$ é ortogonal ao tangente $\psi^{\prime}(s)$. Por outro lado,

$$
\left(\psi^{\prime}(s), D u(\psi(s))\right)=-u_{y}(\psi(s)) u_{x}(\psi(s))+u_{y}(\psi(s)) u_{x}(\psi(s))=0
$$

assim vemos que $D u(\psi(s))$ também é ortogonal a $\psi^{\prime}(s)$. Concluímos que $D u(\psi(s))=$ 
$\lambda \nu(s)$, onde $|\lambda|=\|D u(\psi(s))\|$. Escolhendo $\nu$ de modo que $\lambda=\|D u(\psi(s))\|$, temos

$$
\frac{\partial u}{\partial \nu}(\psi(s))=(D u(\psi(s)), \nu(s))=\lambda(\nu(s), \nu(s))=\lambda=\|D u(\psi(s))\| .
$$

Calculando $\frac{\partial^{2} u}{\partial \nu^{2}}(\psi(s))$, obtemos

$$
\begin{aligned}
\frac{\partial^{2} u}{\partial \nu^{2}}(\psi(s)) & =D^{2} u(\psi(s))(\nu, \nu) \\
& =\frac{u_{x}^{2}(\psi(s)) u_{x x}(\psi(s))+2 u_{x}(\psi(s)) u_{y}(\psi(s)) u_{x y}(\psi(s))+u_{y}^{2}(\psi(s)) u_{y y}(\psi(s))}{\|D u(\psi(s))\|^{2}}
\end{aligned}
$$

Por fim obtemos nosso resultado

$$
\begin{aligned}
\frac{\partial^{2} u}{\partial \nu^{2}}(\psi(s))+ & k \frac{\partial u}{\partial \nu}(\psi(s))= \\
= & \frac{\left(u_{y}^{2}(\psi(s)) u_{x x}(\psi(s))-2 u_{x}(\psi(s)) u_{y}(\psi(s)) u_{x y}(\psi(s))\right)\|D u(\psi(s))\|}{\|D u(\psi(s))\|^{3}}+ \\
& +\frac{\left(u_{x}^{2}(\psi(s)) u_{y y}(\psi(s))\right)\|D u(\psi(s))\|}{\|D u(\psi(s))\|^{3}}+\frac{u_{x}^{2}(\psi(s)) u_{x x}(\psi(s))}{\|D u(\psi(s))\|^{2}}+ \\
& \frac{2 u_{x}(\psi(s)) u_{y}(\psi(s)) u_{x y}(\psi(s))+u_{y}^{2}(\psi(s)) u_{y y}(\psi(s))}{\|D u(\psi(s))\|^{2}} \\
= & \frac{u_{y}^{2}(\psi(s)) u_{x x}(\psi(s))-2 u_{x}(\psi(s)) u_{y}(\psi(s)) u_{x y}(\psi(s))+u_{x}^{2}(\psi(s)) u_{y y}(\psi(s))}{\|D u(\psi(s))\|^{2}}+ \\
& +\frac{u_{x}^{2}(\psi(s)) u_{x x}(\psi(s))+2 u_{x}(\psi(s)) u_{y}(\psi(s)) u_{x y}(\psi(s))+u_{y}^{2}(\psi(s)) u_{y y}(\psi(s))}{\|D u(\psi(s))\|^{2}} \\
= & \frac{u_{y}^{2}(\psi(s)) u_{x x}(\psi(s))+u_{x}^{2}(\psi(s)) u_{y y}(\psi(s))+u_{x}^{2}(\psi(s)) u_{x x}(\psi(s))}{\|D u(\psi(s))\|^{2}}+ \\
& +\frac{u_{y}^{2}(\psi(s)) u_{y y}(\psi(s))}{\|D u(\psi(s))\|^{2}} \\
= & \frac{\left(u_{x}^{2}(\psi(s))+u_{y}^{2}(\psi(s))\right)\left(u_{x x}(\psi(s))\right)+\left(u_{x}^{2}(\psi(s))+u_{y}^{2}(\psi(s))\right)\left(u_{y y}(\psi(s))\right)}{\|D u(\psi(s))\|^{2}} \\
= & \frac{\|D u(\psi(s))\|^{2}}{\|D u(\psi(s))\|^{2}}\left(u_{x x}(\psi(s))+u_{y y}(\psi(s))\right)=\Delta u(\psi(s)) .
\end{aligned}
$$

Observação 1.3. Sejam $\Omega$ um aberto radialmente simétrico em relação a zero e u uma função radial tal que $u(x)=U(\|x\|)$. O teorema acima nos dá a conhecida fórmula

$$
\Delta u(x)=U^{\prime \prime}(\|x\|)+\frac{N-1}{\|x\|} U^{\prime}(\|x\|) .
$$


De fato, sejam $u: \Omega \subset \mathbb{R}^{2} \longrightarrow \mathbb{R}, x \in \Omega,\|x\|=r>0$, tais que $u(y)=U(\|y\|), U$ de classe $\mathcal{C}^{2}$. Localmente, a curva de nível $U(r)$ é uma parte da esfera

$$
S_{r}=\left\{y \in \mathbb{R}^{2} ;\|y\|=r\right\}
$$

quando $D u(x) \neq 0$. A curvatura de $S_{r}$ é constante e vale $\frac{1}{r}$, e o vetor normal é $\nu(y)=\frac{y}{\|y\|}$.

Calculando a derivada normal de $u$ em $x$, temos

$$
\begin{aligned}
\frac{\partial u}{\partial \nu}(x) & =\lim _{t \rightarrow 0} \frac{u(x+t \nu)-u(x)}{t} \\
& =\lim _{t \rightarrow 0} \frac{U(\|x+t \nu\|)-U(\|x\|)}{t} \\
& =\lim _{t \rightarrow 0} \frac{U(\|x\|+t)-U(\|x\|)}{t} \\
& =U^{\prime}(\|x\|) .
\end{aligned}
$$

Por definição $\frac{\partial^{2} u}{\partial \nu^{2}}(x)=D^{2} u(\nu, \nu)=\sum_{i, j=1}^{N} u_{i j}(x) \frac{x_{i} x_{j}}{\|x\|^{2}}$, como $u$ é radial podemos calcular as derivadas parciais

$$
u_{i}(x)=U^{\prime}(x) \frac{x_{i}}{\|x\|}, \quad u_{i j}(x)=U^{\prime \prime}(x) \frac{x_{i} x_{j}}{\|x\|^{2}}+U^{\prime}(x) \frac{\delta_{i j}\|x\|^{2}-x_{i} x_{j}}{\|x\|^{3}} .
$$

Agora vamos calcular a segunda derivada normal

$$
\begin{aligned}
D^{2} u(\nu, \nu) & =\sum_{i, j=1}^{N} U^{\prime \prime}(x) \frac{x_{i}^{2} x_{j}^{2}}{\|x\|^{4}}+U^{\prime}(x)\left(\sum_{i, j=1}^{N} \delta_{i j} \frac{x_{i} x_{j}}{\|x\|^{3}}-\sum_{i, j=1}^{N} \frac{x_{i}^{2} x_{j}^{2}}{\|x\|^{5}}\right) \\
& =U^{\prime \prime}(x)+U^{\prime}(x)\left(\frac{1}{\|x\|}-\frac{1}{\|x\|}\right)=U^{\prime \prime}(x) .
\end{aligned}
$$

Deste modo $\frac{\partial^{2} u}{\partial \nu^{2}}(x)=U^{\prime \prime}(\|x\|)$, e (1.10) se torna (1.11).

Para uma função radial $u: \Omega \subset \mathbb{R}^{N} \longrightarrow \mathbb{R}, N \geq 3$, considere $x \in \Omega, r=\|x\|>0$, com $D u(x) \neq 0$ e

$$
S_{r}^{N}=\left\{y \in \mathbb{R}^{N} ;\|y\|=r\right\}
$$

como antes, $u^{-1}(\|x\|) \cap B_{R}=S_{r}^{N} \cap B_{R}$, onde $B_{R}=B_{R}(x), R>0$.

Considere uma base ortonormal de $\mathbb{R}^{N}$ da seguinte forma $\left\{\nu(x), v_{2}(x), \ldots, v_{N}(x)\right\}$, onde os vetores $v_{i}(x), 1<i \leq N$ são tangentes a $S_{R}$ em $x$, e $\nu(x)=\frac{x}{\|x\|}$ é o vetor normal.

O plano $\pi_{i}=\left\{a \nu(x)+b v_{i}(x) ; a, b \in \mathbb{R}\right\}$ define uma aplicação $u_{i}: \pi_{i} \cap \Omega \longrightarrow \mathbb{R}$, para 
$1<i \leq N, u_{i}=\left.u\right|_{\pi_{i} \cap \Omega}$, isto é,

$$
u_{i}(a, b)=u\left(a \nu(x)+b v_{i}(x)\right)
$$

As funções $u_{i}$ cumprem

$$
\Delta u_{i}(a, b)=\frac{\partial^{2} u}{\partial \nu^{2}}\left(a \nu(x)+b v_{i}(x)\right)+\frac{\partial^{2} u}{\partial v_{i}^{2}}\left(a \nu(x)+b v_{i}(x)\right)
$$

e em particular

$$
\Delta u_{i}(r, 0)=\frac{\partial^{2} u}{\partial \nu^{2}}(x)+\frac{\partial^{2} u}{\partial v_{i}^{2}}(x)
$$

Note agora que $S_{r}^{N} \cap \pi_{i}=S_{r}$, assim podemos aplicar a identidade (1.10) em $u_{i}$,

$$
\Delta u_{i}=\frac{\partial^{2} u}{\partial \nu^{2}}+k \frac{\partial u}{\partial \nu}
$$

De fato,

$$
\|(a, b)\|^{2}=a^{2}+b^{2}=\|a \nu(x)\|^{2}+\left\|b v_{i}(x)\right\|^{2}=\left\|a \nu(x)+b v_{i}(x)\right\|^{2},
$$

por ortogonalidade. Assim, $(a, b) \in S_{r}$ se, e somente se, $a \nu(x)+b v_{i}(x) \in S_{r}^{N}$, e além disso

$$
u_{i}(a, b)=u\left(a \nu(x)+b v_{i}(x)\right)=U\left(\left\|a \nu(x)+b v_{i}(x)\right\|\right)=U\|(a, b)\|,
$$

e portanto $u_{i}$ é radial.

Comparando (1.13) e (1.11) vemos que

$$
\frac{\partial^{2} u}{\partial v_{i}^{2}}(x)=k \frac{\partial u}{\partial \nu}=\frac{1}{r} U^{\prime}(r)
$$

com igualdade para cada $i \in\{2, \ldots, N\}$. Aplicando (1.14) podemos calcular

$$
\begin{aligned}
\Delta u(x) & =\frac{\partial^{2} u}{\partial \nu^{2}}(x)+\sum_{i=1}^{N} \frac{\partial^{2} u}{\partial v_{i}^{2}}(x) \\
& =U^{\prime \prime}(r)+(N-1) \frac{1}{r} U^{\prime}(r) \\
& =U^{\prime \prime}(r)+\frac{N-1}{r} U^{\prime}(r),
\end{aligned}
$$

como queríamos. 


\subsection{Caso bidimensional}

Em dimensão dois podemos considerar um modelo análogo para uma placa fina e plana, à qual é aplicada uma carga de forças verticais. Permitindo que os pontos na extremidade da placa se movam horizontalmente, não haverá aumento da área da superfície da placa. Desprezando então a energia elástica devida à dilatação da placa, um modelo aproximado para a energia mecânica da placa é

$$
J(u)=\int_{\Omega}\left(\frac{1}{2}(\Delta u)^{2}+(1-\sigma)\left(u_{12}^{2}-u_{11} u_{22}\right)-f u\right) d x
$$

onde $\Omega \subset \mathbb{R}^{2}$ é um aberto que representa a placa no estado sem a ação da carga, $f$ : $\Omega \longrightarrow \mathbb{R}$ é a força aplicada, $u: \bar{\Omega} \longrightarrow \mathbb{R}$ é a variação vertical em relação ao equilíbrio e $\sigma$ é uma contante relacionada ao material constituinte da placa, chamada razão de Poisson. Para metais o valor de $\sigma$ é aproximadamente 0.3 e usualmente $\sigma \in(0,0.5)$, mas em geral temos $-1<\sigma<1$; veja [12, p. 5].

O gráfico de $u$ é uma superfície. No lugar do termo representando a curvatura da viga em (1.2), temos

$$
\frac{1}{2}(\Delta u)^{2}+(1-\sigma)\left(u_{12}^{2}-u_{11} u_{22}\right) \approx \frac{1}{2}\left(k_{1}+k_{2}\right)^{2}+(1-\sigma) k_{1} k_{2}
$$

onde $k_{1}$ e $k_{2}$ são as curvaturas principais do gráfico de $u, \frac{k_{1}+k_{2}}{2}$ é a curvatura média e $k_{1} k_{2}$ é a curvatura Gaussiana; para mais detalhes veja [8, Definition 6, p. 146]. Para a aproximação acima assumimos que $\|D u\|$ é pequeno, isto é, $\|D u\| \approx 0$.

A busca de pontos críticos deste funcional nos leva a investigar a equação

$$
J^{\prime}(u) v=0, \forall v \in \mathcal{H}
$$

onde $\mathcal{H}$ é um espaço de Hilbert apropriado onde $J$ está bem definido e que também depende das condições de contorno. Além disso, verifica-se que

$$
J^{\prime}(u) v=\int_{\Omega}\left(\Delta u \Delta v+(1-\sigma)\left(2 u_{12} v_{12}-u_{11} v_{22}-u_{22} v_{11}\right)-f v\right) d x .
$$

Como consideramos apenas casos em que as bordas da placa estão fixadas verticalmente, podemos assumir que $u$ e $v$ se anulam sobre a fronteira de $\Omega$, pois $u$ e $v$ estão no 
mesmo espaço de funções. Temos então

$$
\begin{aligned}
\int_{\Omega}(\Delta u \Delta v) d x & =\int_{\partial \Omega}\left(\Delta u \frac{\partial v}{\partial \nu}\right) d S-\int_{\Omega}(D(\Delta u) D v) d x \\
& =\int_{\partial \Omega}\left(\Delta u \frac{\partial v}{\partial \nu}\right) d S-\int_{\partial \Omega}\left(\frac{\partial}{\partial \nu}(\Delta u) v\right) d S+\int_{\Omega}\left(\Delta^{2} u v\right) d x \\
& =\int_{\partial \Omega}\left(\Delta u \frac{\partial v}{\partial \nu}\right) d S+\int_{\Omega}\left(\Delta^{2} u v\right) d x
\end{aligned}
$$

Por outro lado, empregando o Teorema 1.2, obtemos

$$
\begin{aligned}
\int_{\Omega}\left(2 u_{12} v_{12}-u_{11} v_{22}-u_{22} v_{11}\right) d x= & \int_{\partial \Omega}\left(u_{12} v_{1} \nu_{2}+u_{12} v_{2} \nu_{1}-u_{11} v_{2} \nu_{2}-u_{22} v_{1} \nu_{1}\right) d S \\
& -\int_{\Omega}\left(u_{122} v_{1}+u_{112} v_{2}-u_{112} v_{2}-u_{122} v_{1}\right) d x \\
= & \int_{\partial \Omega}\left(u_{12} v_{1} \nu_{2}+u_{12} v_{2} \nu_{1}-u_{11} v_{2} \nu_{2}-u_{22} v_{1} \nu_{1}\right) d S \\
= & \int_{\partial \Omega}\left(\left(u_{12} \nu_{1} \nu_{2}+u_{12} \nu_{1} \nu_{2}-u_{11} \nu_{2}^{2}-u_{22} \nu_{1}^{2}\right)\|D v\|\right) d S \\
= & \int_{\partial \Omega}\left(\left(2 u_{12} \nu_{1} \nu_{2}-u_{11} \nu_{2}^{2}-u_{22} \nu_{1}^{2}\right) \frac{\partial v}{\partial \nu}\right) d S \\
= & -\int_{\partial \Omega}\left(\left(u_{11}\left(\nu_{1}^{2}+\nu_{2}^{2}\right)+u_{22}\left(\nu_{1}^{2}+\nu_{2}^{2}\right)-u_{11} \nu_{1}^{2}+\right.\right. \\
& \left.\left.-u_{22} \nu_{2}^{2}-2 u_{12} \nu_{1} \nu_{2}\right) \frac{\partial v}{\partial \nu}\right) d S \\
= & -\int_{\Omega}\left(\Delta u-\frac{\partial^{2} u}{\partial \nu^{2}}\right) \frac{\partial v}{\partial \nu} d S \\
= & -\int_{\Omega}\left(k \frac{\partial u}{\partial \nu} \frac{\partial v}{\partial \nu}\right) d S .
\end{aligned}
$$

Para $u=v=0$ em $\partial \Omega$, temos então $\frac{\partial v}{\partial \nu}=\|D v\| \mathrm{e}$

$$
J^{\prime}(u) v=\int_{\Omega}\left(\Delta^{2} u-f\right) v d x+\int_{\partial \Omega}\left(\Delta u-(1-\sigma) k \frac{\partial u}{\partial \nu}\right) \frac{\partial v}{\partial \nu} d S
$$

Para a placa engastada, assumimos também que $\frac{\partial u}{\partial \nu}$ se anula sobre $\partial \Omega$, e podemos supor o mesmo para $\frac{\partial v}{\partial \nu}$. Com isso a integral sobre a fronteira de $\Omega$ em (1.19) se anula, e chegamos a

$$
\int_{\Omega}\left(\Delta^{2} u-f\right) v d x=0, \quad \forall v \in \mathcal{H}
$$

$\operatorname{com} v=\frac{\partial v}{\partial \nu}=0$ sobre $\partial \Omega$. 
Isso nos permite concluir que se um ponto crítico de $J$ for de classe $\mathcal{C}^{4}(\bar{\Omega})$, será uma solução do problema com condições de contorno de Dirichlet

$$
\left\{\begin{array}{rlr}
\Delta^{2} u & =f(x) & \text { em } \Omega \\
u & =\frac{\partial u}{\partial \nu}=0 & \text { em } \partial \Omega
\end{array}\right.
$$

Se por outro lado a placa estiver articulada, não temos a condição sobre $\frac{\partial v}{\partial \nu}$, e o termo de fronteira nos fornece a última condição de contorno que é

$$
\Delta u-(1-\sigma) k \frac{\partial u}{\partial \nu}=0 \text { em } \partial \Omega
$$

Assim, um ponto crítico de classe $\mathcal{C}^{4}(\bar{\Omega})$ é uma solução do seguinte problema de Steklov

$$
\left\{\begin{array}{cccc}
\Delta^{2} u & = & & \text { em } \Omega \\
u & =\Delta u-(1-\sigma) k \frac{\partial u}{\partial \nu}=0 & & \text { em } \partial \Omega
\end{array}\right.
$$

Para mais detalhes sobre a interpretação da condição de contorno de Steklov, veja [14, p. 412].

Se assumirmos que a curvatura da fronteira do aberto $\Omega$ não contribui para a energia ou que $\sigma=1$, obtemos a condição de contorno $\Delta u=0$ em $\partial \Omega$, que nos dá o problema com condição de Navier

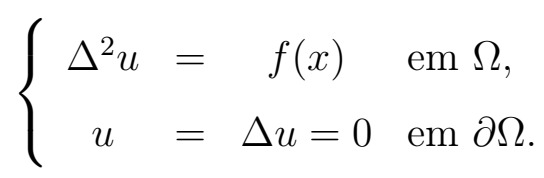




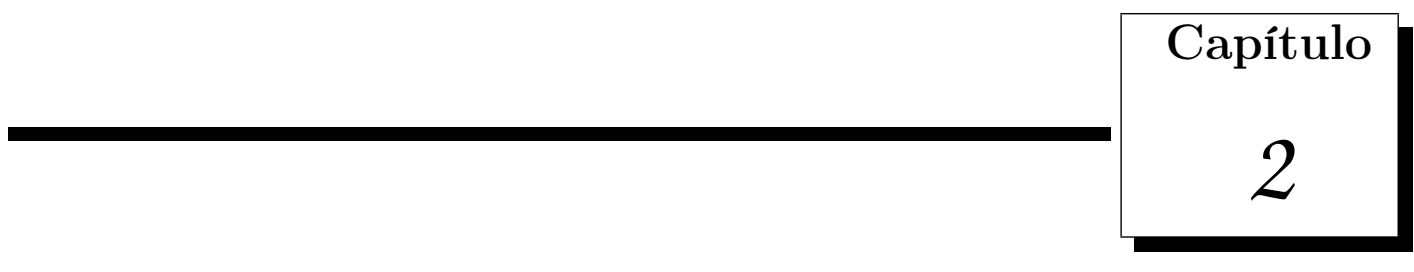

\section{Problemas lineares}

Neste capítulo estudamos a equação

$$
\Delta^{2} u=f(x)
$$

onde a função $f$ é conhecida e $u$ é a incógnita, complementada por uma condição de contorno homogênea. Na Seção 2.5 estudamos os autovalores do operador biharmônico sob cada condição de contorno.

Considere inicialmente o problema linear com condição de Dirichlet

$$
\left\{\begin{array}{rlr}
\Delta^{2} u & =f(x) & \text { em } \Omega \\
u & =\frac{\partial u}{\partial \nu}=0 & \text { em } \partial \Omega
\end{array}\right.
$$

no aberto $\Omega \subset \mathbb{R}^{N}$ limitado, onde $\nu$ é o vetor normal exterior à fronteira de $\Omega$ e a função $f: \Omega \longrightarrow \mathbb{R}$ é dada.

Outras condições de contorno consideradas serão

$$
\left\{\begin{array}{ccc}
\Delta^{2} u & =f(x) & \text { em } \Omega \\
u & =\Delta u=0 & \text { em } \partial \Omega
\end{array}\right.
$$

chamado problema com condição de Navier, e

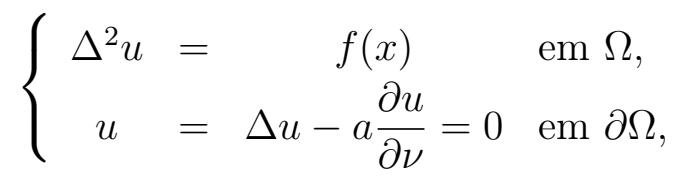


a condição de Steklov, onde $a: \partial \Omega \longrightarrow \mathbb{R}$.

Uma solução clássica para o problema (2.2), ou (2.3) ou (2.4) é uma função $u \in$ $\mathcal{C}^{4}(\bar{\Omega})$ satisfazendo a equação diferencial pontualmente em $\Omega$ e as respectivas condições de contorno em cada ponto de $\partial \Omega$.

Vamos definir a noção de solução fraca para estes problemas. Para isso, suponha que $u \in \mathcal{C}^{4}(\bar{\Omega})$ satisfaz

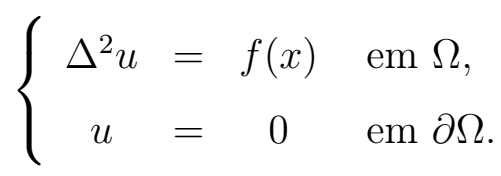

Multiplicando a primeira equação por uma função $v \in \mathcal{C}^{2}(\bar{\Omega})$ que se anula em $\partial \Omega$, isto é, $v \in \mathcal{C}^{2}(\bar{\Omega}) \cap \mathcal{C}_{0}^{0}(\bar{\Omega})$ e integrando em $\Omega$, obtemos

$$
\int_{\Omega}\left(\Delta^{2} u-f\right) v d x=0
$$

Como $u$ também se anula na fronteira de $\Omega$, duas integrações por partes na equação nos levam a

$$
\begin{aligned}
0 & =\int_{\Omega}\left(\Delta^{2} u v-f v\right) d x \\
& =\int_{\Omega}(-(D(\Delta u), D v)-f v) d x \\
& =\int_{\partial \Omega}-\Delta u \frac{\partial v}{\partial \nu} d S+\int_{\Omega}(\Delta u \Delta v-f v) d x .
\end{aligned}
$$

Se $u$ é uma solução clássica de (2.2), então assumimos também que a função teste $v$ satisfaz $\frac{\partial v}{\partial \nu}=0$ em $\partial \Omega$, e o termo de fronteira em (2.5) se anula. Além disso, a integral sobre $\Omega$ é bem definida para

$$
u, v \in H_{0}^{2}(\Omega), \quad f \in L^{2}(\Omega)
$$

Definição 2.1. Seja $f \in L^{2}(\Omega)$. Uma solução fraca de (2.2) é uma função $u \in H_{0}^{2}(\Omega)$ tal que

$$
\int_{\Omega}(\Delta u \Delta v-f v) d x=0, \quad \forall v \in H_{0}^{2}(\Omega)
$$

Para uma solução clássica de (2.3), temos $\Delta u=0$ em $\partial \Omega$, logo o termo de bordo de (2.5) também se anula. Observando novamente que a integral é bem definida para $u, v \in H^{2}(\Omega)$ e $f \in L^{2}(\Omega)$ chegamos à seguinte definição de solução fraca.

Definição 2.2. Uma solução fraca de (2.3) é uma $u \in H^{2}(\Omega) \cap H_{0}^{1}(\Omega)$, tal que para toda 
$v \in H^{2}(\Omega) \cap H_{0}^{1}(\Omega)$,

$$
\int_{\Omega}(\Delta u \Delta v-f v) d x=0
$$

Para o problema de Steklov (2.4), usando a última condição de contorno obtemos

$$
\int_{\Omega}(\Delta u \Delta v-f v) d x-\int_{\partial \Omega} a \frac{\partial u}{\partial \nu} \frac{\partial v}{\partial \nu} d S=0
$$

Suponha que $\partial \Omega$ tem classe $\mathcal{C}^{2}$, de modo que o vetor normal $\nu$ é contínuo e diferenciável. Para $v \in H^{2}(\Omega), 1 \leq i \leq N$, temos $\frac{\partial v}{\partial x_{i}} \in H^{1}(\Omega)$. Logo é bem definido $T\left(\frac{\partial v}{\partial x_{i}}\right) \in L^{2}(\partial \Omega)$ e definimos

$$
\frac{\partial v}{\partial \nu}=\sum_{i=1}^{N} \nu_{i} T\left(\frac{\partial v}{\partial x_{i}}\right)
$$

onde $T$ é o operador traço do Teorema A.7 e $\nu_{i}$ é a i-ésima coordenada de $\nu$.

Definição 2.3. Uma solução fraca do problema (2.4) é uma $u \in H^{2}(\Omega) \cap H_{0}^{1}(\Omega)$, tal que

$$
\int_{\Omega}(\Delta u \Delta v-f v) d x-\int_{\partial \Omega} a \frac{\partial u}{\partial \nu} \frac{\partial v}{\partial \nu} d S=0
$$

para toda função $v \in H^{2}(\Omega) \cap H_{0}^{1}(\Omega)$.

Teorema 2.4. Seja $\Omega \subset \mathbb{R}^{N}$ um aberto limitado.

- Se existe uma solução fraca de (2.2), então ela é única.

- Se $\partial \Omega$ é de classe $\mathcal{C}^{2}$ e existe uma solução fraca de (2.3), então ela é única.

Demonstração. Sejam $u, w \in \mathcal{H}$ soluções fracas de (2.2) ou (2.3), onde $\mathcal{H}=H_{0}^{2}(\Omega)$ ou $\mathcal{H}=H^{2}(\Omega) \cap H_{0}^{1}(\Omega)$, respectivamente. Em ambos os casos, veja os teoremas A.11 e A.13, temos

$$
\int_{\Omega}(\Delta(u-w))^{2} d x=\|u-w\|^{2}
$$

Agora aplicando a definição de solução fraca a $v=u-w$, temos

$$
\int_{\Omega} \Delta u \Delta(u-w) d x=\int_{\Omega} f(u-w) d x=\int_{\Omega} \Delta w \Delta(u-w) d x
$$


que nos dá

$$
\int_{\Omega}(\Delta(u-w))^{2} d x=0
$$

Por (2.9), segue que $\|u-w\|=0$ e assim $u=w$ em $\mathcal{H}$.

Para a condição de Steklov o argumento é o mesmo, porém precisamos de hipóteses sobre $a$; veja a Seção 2.4 .

Observação 2.5. Se existir solução clássica para qualquer dos problemas considerados, então necessariamente $f$ deve ser contínua em $\Omega$. Por outro lado, para estudar a existência de soluções fracas podemos considerar uma classe bem maior de funções $f$, conforme observado acima.

Observe que as soluções clássicas também são soluções fracas. Considere o problema (2.2) com $u \in \mathcal{C}^{4}(\bar{\Omega})$ e tome $v \in \mathcal{C}_{c}^{\infty}(\Omega)$. Multiplicando a equação por $v$ e integrando por partes em $\Omega$, obtemos

$$
\int_{\Omega}(\Delta u \Delta v-f v) d x=0, \quad \forall v \in \mathcal{C}_{c}^{\infty}(\Omega)
$$

Agora como $u \in \mathcal{C}^{4}(\bar{\Omega})$ e $u=D u=0$ em $\partial \Omega$, então $u \in H_{0}^{2}(\Omega)$, pois $\Omega$ é limitado. Além disso, definindo $\varphi: H_{0}^{2}(\Omega) \longrightarrow \mathbb{R}$, por $\varphi(v)=\int_{\Omega}(\Delta u \Delta v-f v) d x$ obtemos um funcional linear limitado em $H_{0}^{2}(\Omega)$. De fato, como $H_{0}^{2}(\Omega)$ está imerso em $L^{2}(\Omega)$ continuamente,

$$
|\varphi v| \leq\|u\|\|v\|+\|f\|_{2}\|v\|_{2} \leq C\|v\|
$$

Como $\varphi$ zera no subespaço denso $\mathcal{C}_{c}^{\infty}(\Omega)$, segue $\varphi \equiv 0$ em $H_{0}^{2}(\Omega)$. Mas então $u$ é uma solução fraca.

Reciprocamente, provada a existência de solução fraca para $f \in L^{2}(\Omega)$, o passo seguinte é verificar se a solução fraca obtida é também uma solução clássica quando $f$ é regular. Este é um problema usualmente chamado de resultado de regularidade. Por hora suponha que $u \in H_{0}^{2}(\Omega)$ seja uma solução fraca de $(2.2)$ com $f \in \mathcal{C}^{0, \alpha}(\bar{\Omega})$, para algum $0<\alpha<1$ e que $u \in \mathcal{C}^{4}(\bar{\Omega})$. Temos pelo Teorema A.7 que a continuidade de $u$ nos dá $u=\frac{\partial u}{\partial \nu}=0$ em $\partial \Omega$. Por contradição, suponha que exista $x \in \Omega \operatorname{com} \Delta^{2} u(x)>f(x)$. Como $u \in \mathcal{C}^{4}(\bar{\Omega})$, temos que $\Delta^{2} u \in \mathcal{C}^{0}(\bar{\Omega})$ e como $f$ é contínua, vai existir $A \subset \Omega$ aberto com $\Delta^{2} u-f>0$ em $A$. Tome $\varphi \in \mathcal{C}_{c}^{\infty}(\Omega)$, com supp $\varphi \subset A, \varphi \geq 0$ em $A, \varphi=1$ em uma bola $B_{r}(x)$ de centro $x$ e raio $r>0$. Integrando, temos

$$
\int_{\Omega}(\Delta u \Delta \varphi-f \varphi) d x=\int_{\Omega}\left(\Delta^{2} u-f\right) \varphi d x=\int_{A}\left(\Delta^{2} u-f\right) \varphi d x \geq \int_{B_{r}(x)}\left(\Delta^{2} u-f\right) d x>0
$$


Como $\mathcal{C}_{c}^{\infty}(\Omega)$ está imerso em $H_{0}^{2}(\Omega)$, u não é uma solução fraca de $(2.2)$, o que é uma contradição.

Analogamente, podemos mostrar que não existe $x \in \Omega$ tal que $\Delta^{2} u(x)<f(x)$. Assim, $\Delta^{2} u(x)=f(x)$ para todo $x \in \Omega$ e $u=\frac{\partial u}{\partial \nu}=0$ sobre $\partial \Omega$. Portanto $u$ é uma solução clássica de $(2.2)$.

Para definir o que entendemos por preservação de positividade, considere o problema de valor de contorno linear

$$
\left\{\begin{aligned}
\Delta^{2} u & =f, \quad \text { em } \Omega \\
\mathcal{B} u & =0, \quad \text { em } \partial \Omega
\end{aligned}\right.
$$

onde $\mathcal{B} u$ é uma das condições de contorno consideradas neste capítulo.

Definição 2.6. Dizemos que (2.10) tem a propriedade de preservação de positividade se para qualquer $f \geq 0$ a solução $u$ de (2.10) satisfaz $u \geq 0$.

\subsection{A condição de Dirichlet}

Seja $f \in L^{2}(\Omega)$. Como introduzido na Definição 2.1, uma solução fraca de (2.2) é um ponto crítico do funcional

$$
J(u)=\int_{\Omega}\left(\frac{1}{2}(\Delta u)^{2}-f u\right) d x, \quad u \in H_{0}^{2}(\Omega) .
$$

Podemos escrever $J=K-L$, onde

$$
K(u)=\frac{1}{2} \int_{\Omega}(\Delta u)^{2} d x, \quad L(u)=\int_{\Omega} f u d x
$$

Pelo Teorema A.15, $L$ é um funcional linear contínuo em $H_{0}^{2}(\Omega)$. Pelo Exemplo B.16, $L$ é de classe $\mathcal{C}^{2}$.

Por outro lado, pelo Teorema A.11,

$$
K(u)=\frac{1}{2}\|u\|^{2}, \quad u \in H_{0}^{2}(\Omega)
$$

Logo, pelo Exemplo B.18, $K$ é de classe $\mathcal{C}^{2}$. Pela Proposição B.15, $J$ é de classe $\mathcal{C}^{2}$. Vamos provar que $J$ possui um único ponto crítico.

Teorema 2.7. Se $\Omega \subset \mathbb{R}^{N}$ é aberto, limitado e $f \in L^{2}(\Omega)$, então existe uma única solução fraca de (2.2). 
Demonstração. Queremos aplicar o Teorema C.4 ao funcional J. Seja $f \in L^{2}(\Omega)$, temos então

$$
\int_{\Omega} f u d x \leq\|f\|_{2}\|u\|_{2} \leq C\|f\|_{2}\|u\|
$$

pelo Teorema A.15. Temos portanto

$$
J(u) \geq \frac{1}{2}\|u\|^{2}-C\|f\|_{2}\|u\|=\|u\|\left(\frac{1}{2}\|u\|-C\|f\|_{2}\right)
$$

e desta desigualdade obtemos a coercividade de $J$. De fato, dado $M \in \mathbb{N}$ tome $R_{0}=$ $2 C\|f\|_{2}+1>0$. Para $R \geq R_{0}$, temos

$$
\frac{R}{2}-C\|f\|_{2}>\frac{R_{0}}{2}-C\|f\|_{2}=\frac{1}{2}
$$

Assim se $R=\max \left\{2 M, R_{0}\right\}$, então $\|u\|>R$ implica em (2.11) $J(u) \geq M$.

Vamos agora verificar a semicontinuidade inferior por sequências de $J$ na topologia fraca. Tome $\left(u_{k}\right) \in H_{0}^{2}(\Omega)$ e suponha que $u_{k} \rightarrow u \in H_{0}^{2}(\Omega)$. Então, em particular, $L\left(u_{k}\right) \longrightarrow L(u)$. Pela Proposição B.4, $K(u) \leq \liminf _{k \rightarrow \infty} K\left(u_{k}\right)$, e por fim

$$
J(u)=K(u)-L(u) \leq \liminf _{k \rightarrow \infty} K\left(u_{k}\right)-\lim _{k \rightarrow \infty} L\left(u_{k}\right)=\liminf _{k \rightarrow \infty} J\left(u_{k}\right)
$$

Pelo Teorema C.4, existe $u \in H_{0}^{2}(\Omega)$ mínimo global de $J$. Este ponto mínimo é um ponto crítico pela Proposição C.1, e assim é uma solução fraca de (2.2). A unicidade decorre do Teorema 2.4 .

O problema (2.2) não pode ser reescrito como um sistema de equações de ordem dois, e portanto não podemos empregar diretamente muitas das técnicas desenvolvidas para equações de ordem dois para este tipo de problema. Para estudar a questão da preservação de positividade podemos analisar a função de Green, porém precisamos nos restringir a domínios onde ela é conhecida explicitamente.

A questão da propriedade de preservação de positividade está relacionada ao sinal da função de Green, se ela existir. De fato, suponha que existe uma função de Green $G_{\Omega}$ associada ao problema (2.10), conforme a Definição 2.9 abaixo e $\partial \Omega$ é de classe $C^{4, \gamma}$, para algum $\gamma \in(0,1]$. Assim, dada $f \in L^{2}(\Omega)$, a solução $u$ de $(2.10)$ é

$$
u(x)=\int_{\Omega} G_{\Omega}(x, y) f(y) d y
$$


Se a função $G_{\Omega}$ é positiva em $\Omega \times \Omega \backslash\{(x, x) ; x \in \Omega\}$, então dada qualquer $f$ nãonegativa, teremos

$$
u(x)=\int_{\Omega} G_{\Omega}(x, y) f(y) d y \geq 0, \quad \forall x \in \Omega,
$$

o que prova a propriedade de preservação de positividade. Além disso, se $f \neq 0$, então $f>0$ em um conjunto de medida positiva. Se para $x \in \Omega$, tivermos para todo $y \in \Omega \backslash\{x\}$, a desigualdade $G_{\Omega}(x, y)>0$, teremos $u(x)>0$ para todo $x \in \Omega$, pois

$$
u(x)=\int_{\Omega} G_{\Omega}(x, y) f(y) d y>0
$$

Por outro lado, suponha que a função de Green assuma algum valor negativo, isto é, existem $x_{0}, y_{0} \in \Omega, x_{0} \neq y_{0}$, tais que $G_{\Omega}\left(x_{0}, y_{0}\right)<0$. Neste caso (2.10) não tem a propriedade de preservação de positividade. De fato, como $G_{\Omega}$ é contínua em uma vizinhança de $\left(x_{0}, y_{0}\right)$, existe $r>0$ tal que para todo $z \in B_{r}\left(y_{0}\right), G_{\Omega}\left(x_{0}, z\right)<0$, com $r>0$ escolhido de modo que $\left|x_{0}-y_{0}\right|>r$. Tomando uma função $\varphi \in \mathcal{C}_{c}^{\infty}(\Omega)$ tal que $\varphi \geq 0$ e supp $\varphi=B_{r}\left(y_{0}\right)$, obtemos um par $(u, \varphi)$ satisfazendo $(2.10)$, com $\varphi \geq 0$ e

$$
u\left(x_{0}\right)=\int_{\Omega} G_{\Omega}\left(x_{0}, z\right) \varphi(z) d z=\int_{B_{r}(y)} G_{\Omega}\left(x_{0}, z\right) \varphi(z) d z<0 .
$$

Como $\varphi \in \mathcal{C}_{c}^{\infty}(\Omega)$, por [12, Theorem 2.19] temos $u \in \mathcal{C}^{4, \gamma}(\Omega)$, em particular $u$ é contínua em uma vizinhança de $x_{0}$. Concluímos que $u$ muda de sinal, logo $\Delta^{2}$ não preserva positividade neste caso.

\subsection{A função de Green do operador biharmônico sob a condição de Dirichlet}

Vamos definir a função de Green para o operador biharmônico em um determinado domínio $\Omega \subset \mathbb{R}^{N}$ sob condição de contorno de Dirichlet. Para isto, vamos definir a solução fundamental para o biharmônico.

Definição 2.8. A solução fundamental para o operador $\Delta^{2} e ́$

$$
F_{N}(x)= \begin{cases}C_{N}\|x\|^{(4-N)}, & \text { se } N \notin\{2,4\}, \\ C_{N}\|x\|^{(4-N)}(-\log (\|x\|), & \text { se } N=2, \text { ou } N=4,\end{cases}
$$

onde as constantes $C_{N}$ são positivas para $N \neq 2$ e $C_{2}<0$; veja [12, p. 48 e p. 121]. 
A solução fundamental cumpre $\Delta^{2} F_{N}(x)=0$ para todo $x \neq 0$ em $\mathbb{R}^{N}$ e é de classe $\mathcal{C}^{\infty}\left(\mathbb{R}^{N} \backslash\{0\}\right)$. Definimos a função de Green através desta solução fundamental.

Definição 2.9. A função de Green de $\Delta^{2}$ em $\Omega$ é uma função

$$
G_{\Omega}: \bar{\Omega} \times \bar{\Omega} \backslash\{(x, x) ; x \in \bar{\Omega}\} \longrightarrow \mathbb{R}
$$

tal que $G_{\Omega}(x, y)=F_{N}(\|x-y\|)+h(x, y)$ e $h(x, \cdot)$ é a solução do problema

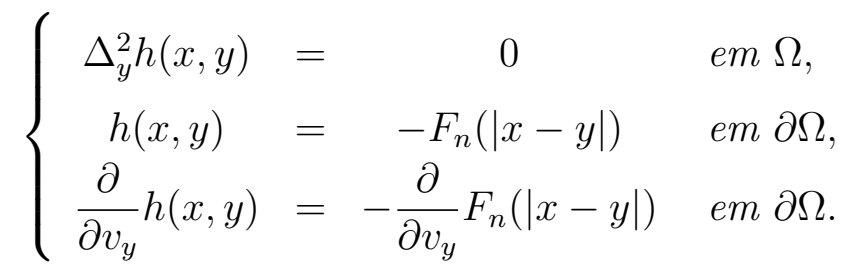

Aqui $\frac{\partial}{\partial \nu_{y}} F(x, y)=\sum_{i=N+1}^{2 N} F_{i}(x, y) \nu_{i}(y)$ denota a derivada na direção normal à fronteira de $\Omega$ da função $F(x, \cdot)$ no ponto $y$. Do mesmo modo, $\Delta_{y} F(x, y)=\sum_{i=N+1}^{2 N} F_{i i}(x, y)$.

Lembramos que o espaço $C^{k, \gamma}(\Omega)$ é o conjunto das funções em $C^{k, \gamma}(\Omega)$ tais que todas as suas derivadas de ordem até $k$ são Hölder-contínuas com expoente $0<\gamma \leq 1$, veja [10, p. 240].

Proposição 2.10. Se $\Omega \subset \mathbb{R}^{N}$ é um aberto limitado com fronteira $\mathcal{C}^{4, \gamma}$, então

$$
G_{\Omega} \in \mathcal{C}^{4, \gamma}(\bar{\Omega} \times \bar{\Omega} \backslash\{(x, x) ; x \in \bar{\Omega}\})
$$

Demonstração. Veja [12, Proposition 4.7].

Em geral é impossível determinar a função de Green de um domínio $\Omega$ arbitrário. Nas próximas subseções estudamos dois casos onde a função de Green é conhecida, a bola unitária de $\mathbb{R}^{N}$ e os limaçons de Pascal.

\subsubsection{A função de Green da bola unitária $B$}

O seguinte lema, devido a Boggio [5, p. 126], nos dá a fórmula para a função de Green de $B$, onde $B \subset \mathbb{R}^{N}$ representa a bola unitária aberta de $\mathbb{R}^{N}$.

Lema 2.11. A função de Green para

$$
\left\{\begin{array}{ccc}
\Delta^{2} u & =f & \text { em } B \\
u & =\frac{\partial u}{\partial \nu}=0 & \text { em } \partial B
\end{array}\right.
$$


é positiva, e é dada por

$$
G_{N}(x, y)=K_{N}|x-y|^{4-N} \int_{1}^{[x y]}\left(\nu^{2}-1\right) \nu^{1-N} d \nu
$$

onde a constante $K_{N}$ é positiva e depende somente de $N$ e $[x y]=\frac{|| x\left|y-\frac{x}{|x|}\right|}{|x-y|}$.

Demonstração. Veja [12, Lemma 2.27] e também [5, p. 126].

Note que $g_{N}(t)=\left(t^{2}-1\right) t^{1-N}$ é uma função crescente em $(0, \infty)$, com $g_{N}(1)=0$. Defina $[x y]=\frac{|| x\left|y-\frac{x}{|x|}\right|}{|x-y|}$. Assim, se $[x y]>1$, temos a integral que define $G_{N}$ calculada sobre um intervalo onde $g_{N}$ é positiva e portanto

$$
G_{N}(x, y)>0 \text {. }
$$

Por outro lado, se $0<[x y]<1$, então $g_{N}$ é negativa, enquanto

$$
\int_{1}^{[x y]} g_{N}(\nu) d \nu=-\int_{[x y]}^{1} g_{N}(\nu) d \nu>0
$$

e portanto $G_{N}(x, y)>0$. Por fim se $[x y]=1$, a integral é nula assim como $G_{N}(x, y)$, e concluímos que

$$
G_{N}(x, y) \geq 0, \quad \forall x, y \in B, x \neq y
$$

\subsubsection{A função de Green dos limaçons de Pascal}

Considere agora a família de domínios

$$
\Omega_{a}=\{(\rho \cos \theta-a, \rho \sin \theta) ; 0 \leq \rho \leq 1+2 a \cos \theta\}, \quad a \in[0,1 / 2]
$$

chamados limaçons de Pascal. Quando $a=0$ temos a bola unitária de $\mathbb{R}^{2}$ e quando $a=\frac{1}{2}$ temos o cardióide.

O limaçon $\Omega_{a}$ é a imagem de $B$ pela transformação conforme $\eta_{a}: B \longrightarrow \Omega_{a}$, definida por

$$
\eta_{a}(x)=x+a x^{2}
$$


ou em coordenadas cartesianas

$$
\eta_{a}\left(x_{1}, x_{2}\right)=\left(x_{1}+a\left(x_{1}^{2}-x_{2}^{2}\right), x_{2}+2 a x_{1} x_{2}\right) .
$$

A função de Green em $\Omega_{a}$ é definida através de $\eta_{a}$,

$$
G_{\Omega_{a}}\left(\eta_{a}(x), \eta_{a}(y)\right)=G_{a}(x, y)
$$

onde $G_{a}: B \times B \backslash\{(x, x) ; x \in B\} \longrightarrow \mathbb{R}$ é dada por

$$
G_{a}(x, y)=\frac{a^{2} s^{2} r^{2}}{16 \pi}\left(\frac{R^{2}}{r^{2}}-1-\log \frac{R^{2}}{r^{2}}-\frac{a^{2}}{1-2 a^{2}} \frac{r^{2}}{s^{2}}\left(\frac{R^{2}}{r^{2}}-1\right)^{2}\right)
$$

e $r=\|x-y\|, R=\left(\left(1-x_{1} y_{1}-x_{2} y_{2}\right)^{2}+\left(x_{2} y_{1}-x_{1} y_{2}\right)^{2}\right)^{\frac{1}{2}}$ e $s=\left(\left(x_{1}+y_{1}+\frac{1}{a}\right)^{2}+\left(x_{2}+y_{2}\right)^{2}\right)^{\frac{1}{2}}$.

Para uma prova de que $G_{a}$ define a função de Green de $\Delta^{2}$ em $\Omega_{a}$, veja [9, p. 10].

Lema 2.12. Sejam $p, q \in B$, tais que $p \neq q$ e $0<a<b \leq \frac{1}{2}$.

- Se $G_{a}(p, q)<0$, então $G_{b}(p, q)<0$.

- $S e G_{b}(p, q)>0$, então $G_{a}(p, q)>0$.

Demonstração. Observe inicialmente que $r$ e $R$ não dependem de $a$ e

$$
s^{2}(a)=\left(p_{1}+q_{1}+\frac{1}{a}\right)^{2}+\left(p_{2}+q_{2}\right)^{2} .
$$

Sejam $p, q \in B, p \neq q$ tais que $G_{a}(p, q)<0$. Trocando por constantes o que não depende de $a$ nesta desigualdade, obtemos

$$
\begin{gathered}
a^{2} s^{2}(a) C\left(D-\frac{a^{2}}{1-2 a^{2}} \frac{1}{s^{2}(a)} E\right)<0 \\
D<\frac{a^{2}}{1-2 a^{2}} \frac{1}{s^{2}(a)} E \\
\frac{D}{E}<\frac{a^{2}}{1-2 a^{2}} \frac{1}{s^{2}(a)}
\end{gathered}
$$

onde $a^{2} s^{2}(a) C$ foi simplificado por ser positivo.

Denotando $M(b)=\frac{b^{2}}{1-2 b^{2}} \frac{1}{s^{2}(b)}$, queremos mostrar que para $b>a, M(b)>\frac{D}{E}$. 
Calculando a derivada de M, obtemos

$$
\begin{aligned}
M^{\prime}(a) & =\frac{2 a}{\left(1-2 a^{2}\right)^{2}} \frac{1}{s^{2}(a)}+\frac{a^{2}}{1-2 a^{2}}\left(-2 s^{-3}(a) s^{\prime}(a)\right) \\
& =\frac{2 a}{\left(1-2 a^{2}\right)^{2}} \frac{2}{\left(1-2 a^{2}\right) s^{4}(a)}\left(p_{1}+q_{1}+\frac{1}{a}\right) .
\end{aligned}
$$

Agora $p, q \in B, \operatorname{logo}\|p\|<1,\|q\|<1$ e $\left|p_{1}\right|<1,\left|q_{1}\right|<1$. Como $a \leq \frac{1}{2}$, então $\frac{1}{a} \leq 2$. Assim

$$
p_{1}+q_{1}+\frac{1}{a} \geq 0
$$

e só temos igualdade se $p_{1}=q_{1}=-1$. Neste caso teríamos $p=q=(-1,0)$, mas $p \neq q$, $\operatorname{logo} p_{1}+q_{1}+\frac{1}{a} \geq 0$.

Logo $M$ é crescente para $b \in(0,1 / 2]$ e para $b>a$,

$$
M(b)>M(a)>\frac{D}{E}
$$

Esta desigualdade implica $G_{b}(p, q)<0$.

Para o segundo item, se $G_{b}(p, q)>0$, procedendo como em (2.17) chegamos a

$$
M(b)<\frac{D}{E}
$$

Como $M$ é crescente, dado qualquer $a$ em $(0,1 / 2]$, se $a<b$, então

$$
\frac{D}{E}>M(b)>M(a)
$$

e concluímos $G_{a}(p, q)>0$.

Teorema 2.13. Existe $a_{0} \in(0,1 / 2)$, tal que $G_{\Omega_{a}}$ é positiva se, e somente se, $a \leq a_{0}$.

Demonstração. Seja $A=\left\{t \in[0,1 / 2]\right.$; a função de Green é postiva em $\left.\Omega_{t}\right\}$. Pela fórmula de Boggio $0 \in A$, enquanto pelo Lema $2.12 A$ é um intervalo fechado. De fato, se $t \in A$, então dados $p, q \in B, p \neq q$, vale $G_{t}(p, q)>0$. Se $s<t$, aplicando o Lema 2.16, $G_{s}(p, q)>0$, e assim $s \in A$, provando que $A$ é um intervalo.

Por outro lado, $W=[0,1 / 2] \backslash A$ é aberto em $[0,1 / 2]$. Se $t \in W$, então existe $p, q \in B$, com $p \neq q$ tais que $G_{t}(p, q)<0$. Se $s>t$, então novamente pelo Lema $2.12 G_{s}(p, q)<0$, e $s \in W$. Como $M$ é contínua, como na demonstração do Lema 2.12 temos

$$
M(t)>\frac{D}{E},
$$


$\operatorname{logo}$ existe $\varepsilon>0$, tal que em $(t-\varepsilon, t+\varepsilon)$ ainda temos $M(s)>\frac{D}{E}$. Logo $(t-\varepsilon, t+\varepsilon) \cap$ $[0,1 / 2] \subset W$, e $W$ é um aberto. Consequentemente $A$ é fechado.

Definindo

$$
a_{0}=\sup A
$$

temos então $A=\left[0, a_{0}\right], W=\left(a_{0}, 1 / 2\right]$.

Tomando $p_{0}=(0.3,0.9), q_{0}=(0.3,-0.9) \in B$ verifica-se que

$$
\frac{R^{2}}{r^{2}}-1-\log \left(\frac{R^{2}}{r^{2}}\right)-\frac{r^{2}}{2 s^{2}}\left(\frac{R^{2}}{r^{2}}-1\right)^{2}<0,
$$

na verdade, aproximadamente -4.74233 , assim $G_{\frac{1}{2}}\left(p_{0}, q_{0}\right)<0$ e $\frac{1}{2} \in W$. Vemos então que $a_{0}<\frac{1}{2}$ e existe um intervalo onde a função de Green de $\Omega_{a}$ muda de sinal.

Para uma prova de que a função de Green em $\Omega_{a}$ é não-negativa para $a \in[0, \sqrt{6} / 6]$ e muda de sinal em $(\sqrt{6} / 6,1 / 2]$; veja $[9$, Theorem 3$]$.

\subsection{A condição de Navier}

Para a condição de Navier consideramos o problema (2.3) com $f \in L^{2}(\Omega)$.

Teorema 2.14. Se $\Omega \subset \mathbb{R}^{N}$ é um aberto limitado com fronteira de classe $\mathcal{C}^{2}$ e $f \in L^{2}(\Omega)$, então existe uma única solução fraca de (2.3).

Demonstração. As soluções fracas de (2.3) são os pontos críticos do funcional $J: H^{2}(\Omega) \cap$ $H_{0}^{1}(\Omega) \longrightarrow \mathbb{R}$ definido por

$$
J(u)=\int_{\Omega}\left(\frac{1}{2}(\Delta u)^{2}-f u\right) d x, \quad u \in H^{2}(\Omega) \cap H_{0}^{1}(\Omega) .
$$

Este funcional é bem definido para $f \in L^{2}(\Omega)$, e pelo Teorema A.13, as hipóteses em $\Omega$ nos dão

$$
J(u)=\frac{1}{2}\|u\|^{2}-\int_{\Omega} f u d x .
$$

Novamente temos a desigualdade

$$
J(u) \geq \frac{1}{2}\|u\|^{2}-\|f\|_{2}\|u\|
$$

e $J$ é coercivo e semicontínuo inferiormente por sequências na topologia fraca de $H^{2}(\Omega) \cap$ 
$H_{0}^{1}(\Omega)$. Pelo Teorema C.4 temos a existência de um ponto mínimo global, que é ponto crítico de $J$, pois $J \in \mathcal{C}^{1}\left(H^{2}(\Omega) \cap H_{0}^{1}(\Omega)\right)$.

Nesta definição de solução fraca não está inclusa a condição $\Delta u=0$ na fronteira de $\Omega$. Ela pode ser verificada reescrevendo (2.3) em forma de um sistema de equações de segunda ordem:

$$
\left\{\begin{array}{l}
-\Delta u=w, \quad-\Delta w=f \quad \text { em } \quad \Omega \\
u=w=0 \quad \text { em } \quad \partial \Omega
\end{array}\right.
$$

Se $f \in L^{2}(\Omega)$, então existe uma única solução fraca para o problema

$$
\left\{\begin{array}{cccc}
-\Delta w & = & \text { em } \Omega \\
w & = & 0 & \text { em } \partial \Omega
\end{array}\right.
$$

isto é, $w \in H_{0}^{1}(\Omega)$ e

$$
\int_{\Omega}(D w D v-f v) d x=0, \quad \forall v \in H_{0}^{1}(\Omega)
$$

veja por exemplo [4].

Agora novamente, como $H_{0}^{1}(\Omega)$ está imerso em $L^{2}(\Omega), w \in L^{2}(\Omega)$ e existe uma única solução fraca $u_{0} \in H_{0}^{1}(\Omega)$ para o segundo problema

$$
\left\{\begin{array}{cccc}
-\Delta u_{0} & = & \text { em } \Omega \\
u_{0} & = & 0 & \text { em } \partial \Omega
\end{array}\right.
$$

Assim existe uma solução $\left(u_{0}, w\right) \in H_{0}^{1}(\Omega) \times H_{0}^{1}(\Omega)$ para o sistema (2.18), que chamaremos de solução de sistema para (2.3). Como $\partial \Omega$ é de classe $\mathcal{C}^{2}$, por [2, Theorem 1.1] $u_{0} \in H^{2}(\Omega)$. Podemos calcular

$$
\begin{aligned}
\int_{\Omega} f v d x & =\int_{\Omega} D w D v d x=\sum_{i=1}^{N} \int_{\Omega} w_{i} v_{i} d x \\
& =-\int_{\Omega} w \sum v_{i i} d x=\int_{\Omega} w(-\Delta v) d x
\end{aligned}
$$


onde foi usada a definição de derivada fraca. Novamente

$$
\begin{aligned}
\int_{\Omega} w(-\Delta v) d x & =\int_{\Omega} D\left(u_{0}\right) D(-\Delta v) d x=\sum_{i=1}^{N} \int_{\Omega}\left(u_{o}\right)_{i}(-\Delta v)_{i} d x \\
& =-\sum_{i=1}^{N} \int_{\Omega}\left(u_{0}\right)_{i i}(-\Delta v) d x=\int_{\Omega} \Delta u_{0} \Delta v d x
\end{aligned}
$$

com as igualdades (2.19) e (2.20) valendo para todo $v \in \mathcal{C}_{c}^{\infty}(\Omega)$. Mas então $u_{0}$ é uma solução fraca, e pela unicidade do Teorema $2.4, u_{0}=u$.

Mas $-\Delta u_{0}=w \in H_{0}^{1}(\Omega)$, logo no sentido do traço $u_{0}$ cumpre $-\Delta u_{0}=0$ em $\partial \Omega$.

A decomposição em sistema também é interessante para mostrar que o problema (2.3) preserva positividade. De fato, sejam $f \in L^{2}(\Omega), f \geq 0$ e $\left(u_{0}, w\right) \in\left(H^{2}(\Omega) \cap H_{0}^{1}(\Omega)\right) \times$ $\left(H^{2}(\Omega) \cap H_{0}^{1}(\Omega)\right)$ a solução de sistema de (2.3), aplicando o Corolário A.19, $w \geq 0$ em $\Omega$, outra aplicação do Corolário A.19 dá $u \geq 0$ em $\Omega$.

\subsection{A condição de Steklov}

Nesta seção consideramos o seguinte problema com condição de contorno homogênea no aberto $\Omega \subset \mathbb{R}^{N}$

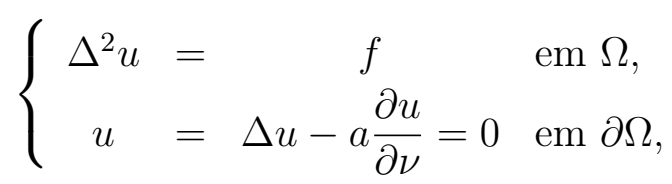

onde $f: \Omega \longrightarrow \mathbb{R}$ e $a: \partial \Omega \longrightarrow \mathbb{R}$ são funções conhecidas.

O teorema a seguir, [12, Theorem 5.22], é o principal resultado desta seção.

Teorema 2.15. Sejam $\Omega \subset \mathbb{R}^{N}$ um aberto limitado com fronteira $\mathcal{C}^{2}, N \geq 2$ e $b: \partial \Omega \longrightarrow$ $\mathbb{R}$ continua e não-negativa. Então existem $\delta_{1, b} \in \mathbb{R}, \delta_{c, b} \in \mathbb{R} \cup\{-\infty\}$, com $\delta_{c, b}<0<\delta_{1, b}$, tais que para toda $a: \partial \Omega \longrightarrow \mathbb{R}$ contínua:

1. Se $a \geq \delta_{1, b} b, f \geq 0, f \neq 0$ em $L^{2}(\Omega)$, então não existe solução fraca não-negativa de $(2.21)$.

2. Se $a=\delta_{1, b}$, então (2.21) com $f=0$ admite uma solução fraca $u_{1, b}$ positiva em $\Omega$, tal $q u e-\Delta u_{1, b}>0$ em $\Omega e \frac{\partial u_{1, b}}{\partial \nu}<0$ em $\partial \Omega$. A menos de múltiplos, $u_{1, b}$ é única.

3. Se $a \leq \delta_{1, b} b$, mas $a \neq \delta_{1, b} b$, então para toda $f \in L^{2}(\Omega)$ existe uma única solução fraca de (2.21). Além disso:

(a) Se $a \geq \delta_{c, b} b$ e $f \geq 0$, então $u \geq 0$. 
(b) Se $a>\delta_{c, b} b$ e $f \geq 0, f \neq 0$, então existe $c_{f}>0$, tal que $u(x) \geq c_{f} \operatorname{dist}(x, \partial \Omega)$. Além disso, $-\Delta u \geq 0$ em $\Omega$ se, e somente se, $a \geq 0$ em $\partial \Omega$.

(c) Se $a<\delta_{c, b}$, então existe $f \in L^{2}(\Omega), f \geq 0, f \neq 0$, tal que a solução u de (2.21) não é não-negativa.

Para provar o Teorema 2.15 precisaremos do Lema 2.16.

Lema 2.16. Dada $a: \partial \Omega \longrightarrow \mathbb{R}$ contínua, $a \leq \delta_{1, b} b, a \neq \delta_{1, b} b$, existe $\varepsilon>0$ tal que para toda $u \in H^{2}(\Omega) \cap H_{0}^{1}(\Omega)$,

$$
\int_{\Omega}(\Delta u)^{2} d x-\int_{\partial \Omega} a\left(\frac{\partial u}{\partial \nu}\right)^{2} d S \geq \varepsilon \int_{\Omega}(\Delta u)^{2} d x
$$

Demonstração. Observe que para toda $u \in H^{2}(\Omega) \cap H_{0}^{1}(\Omega)$, temos

$$
\int_{\Omega}(\Delta u)^{2} d x \geq \delta_{1, b} \int_{\partial \Omega} b\left(\frac{\partial u}{\partial \nu}\right)^{2} d S
$$

isto é,

$$
\int_{\Omega}(\Delta u)^{2} d x-\int_{\partial \Omega} \delta_{1, b} b\left(\frac{\partial u}{\partial \nu}\right)^{2} d S \geq 0 .
$$

Primeiramente observe que (2.22) é trivialmente satisfeita $\operatorname{com} \varepsilon=1$ no caso em que $a \leq 0$ em $\partial \Omega$.

No caso em que $a<\delta_{1, b} b$ e existe $x_{0} \in \partial \Omega$ tal que $0<a\left(x_{0}\right)$, tome

$$
\varepsilon=\frac{\min \left\{\delta_{1, b} b(x)-a(x) ; x \in \partial \Omega\right\}}{\max \left\{\delta_{1, b} b(x) ; x \in \partial \Omega\right\}}>0 .
$$

Observe que $\varepsilon<1$, pois $\delta_{1, b} b\left(x_{0}\right)-a\left(x_{0}\right)<\delta_{1, b} b\left(x_{0}\right)$. Observe também que para todo $x \in \partial \Omega, \delta_{1, b} b(x)-a(x) \geq \varepsilon \delta_{1, b} b(x)$, ou seja, $a \leq(1-\varepsilon) \delta_{1, b} b$. Assim

$$
\begin{gathered}
\int_{\Omega}(\Delta u)^{2} d x-\int_{\partial \Omega} a\left(\frac{\partial u}{\partial \nu}\right)^{2} d S=\varepsilon \int_{\Omega}(\Delta u)^{2} d x+(1-\varepsilon) \int_{\Omega}(\Delta u)^{2} d x-\int_{\partial \Omega} a\left(\frac{\partial u}{\partial \nu}\right)^{2} d S \\
\geq \varepsilon \int_{\Omega}(\Delta u)^{2} d x+(1-\varepsilon) \int_{\Omega}(\Delta u)^{2} d x-\int_{\partial \Omega}(1-\varepsilon) \delta_{1, b} b\left(\frac{\partial u}{\partial \nu}\right)^{2} d S \\
=\varepsilon \int_{\Omega}(\Delta u)^{2} d x+(1-\varepsilon)\left(\int_{\Omega}(\Delta u)^{2} d x-\int_{\partial \Omega} \delta_{1, b} b\left(\frac{\partial u}{\partial \nu}\right)^{2} d S\right) \geq \varepsilon \int_{\Omega}(\Delta u)^{2} d x .
\end{gathered}
$$

Suponha agora que existem $x_{0}, x_{1} \in \partial \Omega \operatorname{com} a\left(x_{0}\right)=\delta_{1, b} b\left(x_{0}\right)$ e $0<a\left(x_{1}\right)<\delta_{1, b} b\left(x_{1}\right)$. 
Defina $\widetilde{b}=\frac{1}{2}\left(b+\frac{a^{+}}{\delta_{1, b}}\right)$. Assim $\widetilde{b}: \partial \Omega \longrightarrow \mathbb{R}$ é contínua, não-negativa,

$$
\widetilde{b}=\frac{1}{2} b+\frac{1}{2} \frac{a^{+}}{\delta_{1, b}} \leq \frac{1}{2} b+\frac{1}{2} b=b \quad \text { em } \quad \partial \Omega
$$

e

$$
\widetilde{b}\left(x_{1}\right)=\frac{1}{2} b\left(x_{1}\right)+\frac{1}{2} \frac{a\left(x_{1}\right)}{\delta_{1, b}}<b\left(x_{1}\right) .
$$

Aplicando a primeira parte do Lema 2.25, existem $u_{1}, u_{2} \in H^{2}(\Omega) \cap H_{0}^{1}(\Omega)$ tais que

$$
u_{1}=\min _{u \in H^{2}(\Omega) \cap H_{0}^{1}} J_{b}(u), \quad u_{2}=\min _{u \in H^{2}(\Omega) \cap H_{0}^{1}} J_{\widetilde{b}}(u) .
$$

Temos ainda

$$
\delta_{1, b}=J_{b}\left(u_{1}\right) \leq J_{b}\left(u_{2}\right)<J_{\widetilde{b}}\left(u_{2}\right)=\delta_{1, \widetilde{b}}
$$

Colocando $\varepsilon=1-\frac{\delta_{1, b}}{\delta_{1, \widetilde{b}}}>0$, temos

$$
a \leq a^{+}=\delta_{1, b}(2 \widetilde{b}-b) \leq \delta_{1, b} \widetilde{b}=(1-\varepsilon) \delta_{1, \widetilde{b}} \widetilde{b}
$$

Por fim,

$$
\begin{aligned}
& \int_{\Omega}(\Delta u)^{2} d x-\int_{\partial \Omega} a\left(\frac{\partial u}{\partial \nu}\right)^{2} d S=\varepsilon \int_{\Omega}(\Delta u)^{2} d x+(1-\varepsilon) \int_{\Omega}(\Delta u)^{2} d x-\int_{\partial \Omega} a\left(\frac{\partial u}{\partial \nu}\right)^{2} d S \\
& \geq \varepsilon \int_{\Omega}(\Delta u)^{2} d x+(1-\varepsilon)\left(\int_{\Omega}(\Delta u)^{2} d x-\int_{\partial \Omega} \delta_{1, \widetilde{b}} \widetilde{b}\left(\frac{\partial u}{\partial \nu}\right)^{2} d S\right) \geq \varepsilon \int_{\Omega}(\Delta u)^{2} d x .
\end{aligned}
$$

Consideremos agora o último caso, isto é, quando $a^{+}=\delta_{1, b} b$ e $a^{-} \neq 0$. Neste caso temos $\widetilde{b}=\frac{1}{2}(b+b)=b$. Vamos substituir $J_{b}$ por

$$
J_{b}^{a^{-}}(u)=\frac{\int_{\Omega}(\Delta u)^{2} d x+\int_{\partial \Omega} a^{-}\left(\frac{\partial u}{\partial \nu}\right)^{2} d S}{\int_{\partial \Omega} b\left(\frac{\partial u}{\partial \nu}\right)^{2} d S}
$$

Definindo $\delta_{1, b}^{a^{-}}$para $J_{b}^{a^{-}}$como em (2.44), o mesmo argumento mostra que existe $u_{1, b}^{a^{-}} \in$ $H^{2} \cap H_{0}^{1}(\Omega)$ que minimiza $J_{b}^{a^{-}}$. Temos então

$$
\delta_{1, b}^{a^{-}}=J_{b}^{a^{-}}\left(u_{1, b}^{a^{-}}\right) \geq J_{b}\left(u_{1, b}^{a^{-}}\right) \geq J_{b}(u-1, b)=\delta_{1, b} .
$$

Como $\frac{\partial u_{1, b}}{\partial \nu} \neq 0$ em $\partial \Omega$, se $u_{1, b}^{a^{-}}=\lambda u_{1, b}$, então a primeira desigualdade é estrita. Por 
outro lado, se não existir $\lambda \in \mathbb{R} \backslash\{0\}$ tal que $u_{1, b}^{a^{-}}=\lambda u_{1, b}$, então a segunda desigualdade é estrita, pois $\delta_{1, b}$ é simples; veja o Lema 2.25. Em qualquer caso, $\delta_{1, b}^{a^{-}}>\delta_{1, b}$. Tome então

$$
\varepsilon=1-\frac{\delta_{1, b}}{\delta_{1, b}^{a-}}
$$

e observe que $0<\varepsilon<1$. Assim,

$$
\begin{aligned}
\int_{\Omega}|\Delta u|^{2} d x-\int_{\partial \Omega} a\left(\frac{\partial u}{\partial \nu}\right)^{2} d S= & \int_{\Omega}|\Delta u|^{2} d x+\int_{\partial \Omega} a^{-}\left(\frac{\partial u}{\partial \nu}\right)^{2} d S-\int_{\partial \Omega} \delta_{1, b} b\left(\frac{\partial u}{\partial \nu}\right)^{2} d S \\
\geq & \int_{\Omega}|\Delta u|^{2} d x+(1-\varepsilon)\left(\int_{\Omega}|\Delta u|^{2} d x+\int_{\partial \Omega} a^{-}\left(\frac{\partial u}{\partial \nu}\right)^{2} d S\right. \\
& \left.-\int_{\partial \Omega} \delta_{1, b}^{a^{-}} b\left(\frac{\partial u}{\partial \nu}\right)^{2} d S\right) \geq \varepsilon \int_{\Omega}|\Delta u|^{2} d x
\end{aligned}
$$

Prova do Teorema 2.15. Seja $\Omega \subset \mathbb{R}^{N}$ um aberto limitado com fronteira $\mathcal{C}^{2}, N \geq 2$.

Prova do item 1. Sejam $a>\delta_{1, b} b, \delta_{1, b}$ definido em (2.44), $f \in L^{2}(\Omega), f \geq 0, f \neq 0$, suponha que $u \in H^{2}(\Omega) \cap H_{0}^{1}(\Omega)$ é uma solução fraca de (2.21), $u \geq 0$, tome $v=u_{1, b}$ minimizador de $J_{b}$. Temos

$$
\begin{gathered}
0<\int_{\Omega} f u_{1, b} d x=\int_{\Omega} \Delta u \Delta u_{1, b} d x-\int_{\partial \Omega} a \frac{\partial u}{\partial \nu} \frac{\partial u_{1, b}}{\partial \nu} d S \\
\leq \int_{\Omega} \Delta u \Delta u_{1, b} d x-\int_{\partial \Omega} \delta_{1, b} b \frac{\partial u}{\partial \nu} \frac{\partial u_{1, b}}{\partial \nu} d S=0,
\end{gathered}
$$

contradição. Note que a primeira igualdade vem da positividade de $u_{1, b}$ no item 2 , e a última porque $u_{1, b}$ é solução fraca de (2.21) com $f=0$ e $a=\delta_{1, b} b$.

Prova do item 2. Será provado no Lema 2.25.

Prova do item 3. Vamos empregar o Teorema C.4 e o Lema 2.16. De fato, como $a \leq \delta_{1, b} b$, $a \neq \delta_{1, b} b$, existe $\varepsilon>0$, tal que

$$
\int_{\Omega}(\Delta u)^{2} d x-\int_{\partial \Omega} a\left(\frac{\partial u}{\partial \nu}\right)^{2} d S \geq \varepsilon \int_{\Omega}(\Delta u)^{2} d x
$$


para toda $u \in H^{2}(\Omega) \cap H_{0}^{1}(\Omega)$. Assim, pela Desigualdade de Hölder (Teorema A.2)

$$
J(u) \geq \varepsilon \int_{\Omega}(\Delta u)^{2} d x-C\|f\|_{2}\|u\|_{2}=\varepsilon\|u\|^{2}-C\|f\|_{2}\|u\|,
$$

pelo Teorema A.13. A desigualdade (2.29) nos permite concluir que $J$ é coercivo. O funcional $J$ é de classe $\mathcal{C}^{1}$, pelos Exemplos B.16 e B.17. Para aplicar o Teorema C.4, vamos mostrar que $J$ é semicontínuo inferiormente por sequências na topologia fraca. Para isso precisaremos da compacidade do operador traço $T$.

Seja $\left(u_{k}\right) \in H^{2}(\Omega) \cap H_{0}^{1}(\Omega)$, tal que $u_{k} \rightarrow u \in H^{2}(\Omega) \cap H_{0}^{1}(\Omega)$, pela Proposição B.4, sabemos que

$$
\liminf _{k \rightarrow \infty}\left\|u_{k}\right\|^{2} \geq\|u\|^{2}
$$

Como o operador traço é compacto [1] e $\left(u_{k}\right)$ é limitada em $H^{2}(\Omega) \cap H_{0}^{1}(\Omega)$, existe uma subsequência ainda denotada $\left(u_{k}\right)$ tal que

$$
1 \leq i \leq N \Longrightarrow T\left(\frac{\partial u_{k}}{\partial x_{i}}\right) \longrightarrow T\left(\frac{\partial u}{\partial x_{i}}\right) \text { em } L^{2}(\partial \Omega)
$$

Assim,

$$
\frac{\partial u_{k}}{\partial \nu}=\sum_{i=1}^{N} T\left(\frac{\partial u_{k}}{\partial x_{i}}\right) \nu_{i} \longrightarrow \frac{\partial u}{\partial \nu} \operatorname{em~} L^{2}(\partial \Omega),
$$

e como $a \in \mathcal{C}(\partial \Omega)$

$$
\int_{\partial \Omega} a\left(\frac{\partial u}{\partial \nu}\right)^{2} d S=\lim _{k \rightarrow \infty} \int_{\partial \Omega} a\left(\frac{\partial u_{k}}{\partial \nu}\right)^{2} d S
$$

pois a limitado, implica $\int_{\partial \Omega} a(\cdot) d S$ é funcional linear contínuo em $L^{1}(\partial \Omega)$. Por fim,

$$
\begin{aligned}
\liminf _{k \rightarrow \infty} J\left(u_{k}\right) & =\liminf _{k \rightarrow \infty} \int_{\Omega}\left(\Delta u_{k}\right)^{2} d x-\liminf _{k \rightarrow \infty} \int_{\partial \Omega} a\left(\frac{\partial u_{k}}{\partial \nu}\right)^{2} d S-\liminf _{k \rightarrow \infty} \int_{\Omega} f u_{k} d x \\
& \geq \liminf _{k \rightarrow \infty}\left\|u_{k}\right\|^{2}-\int_{\Omega} a\left(\frac{\partial u}{\partial \nu}\right)^{2} d S-\lim _{k \rightarrow \infty} \int_{\Omega} f u_{k} d x \geq J(u)
\end{aligned}
$$

Pelo Teorema C.4, existe um ponto crítico de $J$, solução de (2.4). Para provar a unicidade, sejam $u, v \in H^{2}(\Omega) \cap H_{0}^{1}(\Omega)$ duas soluções fracas de (2.4), temos

$$
\int_{\Omega}(\Delta(u-v))^{2} d x-\int_{\partial \Omega} a\left(\frac{\partial(u-v)}{\partial \nu}\right)^{2} d S=0 .
$$


Mas $w=u-v \in H^{2}(\Omega) \cap H_{0}^{1}(\Omega)$, logo usando $(2.23)$ e $a<\delta_{1, b} b$, temos

$$
\int_{\Omega}(\Delta(w))^{2} d x-\int_{\partial \Omega} a\left(\frac{\partial(w)}{\partial \nu}\right)^{2} d S \geq \int_{\Omega}(\Delta(w))^{2} d x-\int_{\partial \Omega} \delta_{1, b} b\left(\frac{\partial(w)}{\partial \nu}\right)^{2} d S>0
$$

se $w \neq \lambda u_{1, b}$, para todo $\lambda \in \mathbb{R} \backslash\{0\}$.

Por outro lado, se $w=\lambda u_{1, b}, \lambda \neq 0$, então

$$
\int_{\Omega}(\Delta(w))^{2} d x=\int_{\partial \Omega} \delta_{1, b} b\left(\frac{\partial(w)}{\partial \nu}\right)^{2} d S
$$

e

$$
\int_{\partial \Omega}\left(\delta_{1, b} b-a\right)\left(\frac{\partial(w)}{\partial \nu}\right)^{2} d S=\lambda^{2} \int_{\partial \Omega}\left(\delta_{1, b} b-a\right)\left(\frac{\partial u_{1, b}}{\partial \nu}\right)^{2} d S>0
$$

pois $a<\delta_{1, b} b$ em um conjunto aberto em $\partial \Omega$, e pelo item $2, \frac{\partial u_{1, b}}{\partial \nu} \neq 0$ em todo o bordo. Mas isto é uma contradição e portanto $w=0$, ou $u=v$.

Para provar o item 3.(a), sejam

$$
\begin{aligned}
A=\{s \in \mathbb{R} ; & \forall f \in L^{2}(\Omega), \forall a \in \mathcal{C}(\partial \Omega), \\
& \text { se } a \geq s b, f \geq 0 \text { e existe } u \text { solução de }(2.4), \text { então } u \geq 0 \text { em } \Omega\},
\end{aligned}
$$

e tome $\delta_{c, b}=\inf A$. Note que $0 \in A$, pois se $u \in H^{2}(\Omega) \cap H_{0}^{1}(\Omega)$ é solução de (2.4) e $f \geq 0, a \geq 0$, então decompondo (2.4) como sistema, o princípio do máximo (Corolário A.19) nos dá

$$
-\Delta u \geq \inf _{\partial \Omega}(-\Delta u) \geq 0
$$

Logo como $u=0$ em $\partial \Omega$, outra aplicação do princípio do máximo leva a

$$
u \geq \inf _{\partial \Omega} u=0
$$

Por outro lado, se $s \in A$ e $\delta_{1, b}>t>s$, então claramente $t \in A$, pois se $a \geq t b>s b$ e $f \geq 0$, então a solução $u$ é não-negativa e $t \in A$. Dessa forma,

$$
A \supset\left(\delta_{c, b}, \delta_{1, b}\right)
$$

onde o ínfimo é tomado como sendo $-\infty$, se $A$ não é limitado inferiormente.

Pela definição de $\delta_{c, b}, a<\delta_{c, b} b$ implica a existência de $f \in L^{2}(\Omega), f \geq 0, f \neq 0$, com a solução de (2.4) $u \ngtr 0$, o que prova o item $(c)$.

Agora se $a>\delta_{c, b} b$ também a solução $u$ de (2.4) será não-negativa para $f \geq 0$, provando 
o item $(a)$.

Para o item (b) veja [12].

A seguir será provado que o problema da placa articulada estudada no Capítulo 1 preserva positividade, isto é, a deformação ocorre na direção que age o peso.

Teorema 2.17. Se $\Omega \subset \mathbb{R}^{2}$ é um aberto limitado e convexo, com fronteira $\mathcal{C}^{2}, f \in L^{2}(\Omega)$ e $\sigma \in(-1,1)$, então existe uma única solução fraca de (1.21) e se $f \geq 0$, então a solução u cumpre $u \geq 0$.

Demonstração. Considere o funcional $J: H^{2}(\Omega) \cap H_{0}^{1}(\Omega) \longrightarrow \mathbb{R}$, definido por (1.16), $J$ é de classe $\mathcal{C}^{1}$, vamos provar que $J$ é coercivo e semincontínuo inferiormente por sequências na topologia fraca. Reescrevendo $J(u)$, obtemos

$$
\begin{aligned}
J(u) & =\int_{\Omega}\left(\frac{1}{2}(\Delta u)^{2}+(1-\sigma)\left(u_{12}^{2}-u_{11} u_{22}\right)-f u\right) d x \\
& =\int_{\Omega}\left(\frac{1}{2}\left(u_{11}^{2}+2 u_{11} u_{22}+u_{22}^{2}\right)+u_{12}^{2}-u_{11} u_{22}-\sigma u_{12}^{2}+\sigma u_{11} u_{22}-f u\right) d x \\
& =\int_{\Omega}\left(\frac{\sigma}{2}\left(u_{11}+u_{22}\right)^{2}+\frac{1-\sigma}{2}\left(u_{11}^{2}+2 u_{12}^{2}+u_{22}^{2}\right)-f u\right) d x
\end{aligned}
$$

Vemos assim que para $\sigma \geq 0$,

$$
J(u) \geq C\|u\|^{2}-D\|f\|_{2}\|u\|,
$$

e assim $J$ é coercivo. Além disso, existe $c>0$ tal que

$$
\begin{aligned}
\frac{\sigma}{2} \int_{\Omega}(\Delta u)^{2} d x & \leq \int_{\Omega}\left(\frac{\sigma}{2}(\Delta u)^{2}+\frac{1-\sigma}{2}\left(u_{11}^{2}+2 u_{12}^{2}+u_{22}^{2}\right)\right) d x \\
& \leq \int_{\Omega}(\Delta x)^{2} d x+2 \int_{\Omega}\left(u_{11}^{2}+u_{12}^{2}+u_{22}^{2}\right) d x \\
& \leq c \int_{\Omega}(\Delta u)^{2} d x .
\end{aligned}
$$

Logo

$$
\left(\int_{\Omega} \frac{\sigma}{2}(\Delta u)^{2} d x+\int_{\Omega} \frac{1-\sigma}{2}\left(u_{11}^{2}+2 u_{12}^{2}+u_{22}^{2}\right) d x\right)^{\frac{1}{2}}
$$

define uma norma em $H^{2}(\Omega) \cap H_{0}^{1}(\Omega)$, equivalente a $\left(\int_{\Omega}(\Delta u)^{2} d x\right)^{\frac{1}{2}}$. Usando esta norma, 
denotada \|\|$_{J}$, o funcional $J$ tem a forma

$$
J(u)=\|u\|_{J}^{2}-\int_{\Omega} f u d x
$$

Como vimos nas provas dos Teoremas 2.7 e 2.14, $J$ é semicontínuo inferiormente por sequências na topologia fraca.

Se $-1<\sigma<0$, então $2 \sigma u_{11} u_{22} \geq \sigma\left(u_{11}^{2}+u_{22}^{2}\right)$, e assim

$$
\begin{aligned}
\frac{1}{2}(\Delta u)^{2}+\frac{1-\sigma}{2}\left(u_{12}^{2}-u_{11} u_{22}\right) & =\frac{1}{2}\left(u_{11}^{2}+u_{22}^{2}\right)+u_{11} u_{22}+\frac{\sigma-1}{2} u_{11}+u_{22}+\frac{1-\sigma}{2} u_{12}^{2} \\
& =\frac{1}{2}\left(u_{11}^{2} u_{22}^{2}\right)+\frac{1-\sigma}{2} u_{12}^{2}+\frac{\sigma}{2} u_{11} u_{22} \\
& \geq \frac{1}{2}\left(u_{11}^{2}+u_{22}^{2}\right)+\frac{1-\sigma}{2} u_{12}^{2}+\frac{\sigma}{4}\left(u_{11}^{2}+u_{22}^{2}\right) \\
& =\frac{2+\sigma}{4}\left(u_{11}^{2}+u_{22}^{2}\right)+\frac{1-\sigma}{2} u_{12}^{2} .
\end{aligned}
$$

Como $2+\sigma>0$ e $1-\sigma>0$, vemos que existe $C>0$, tal que

$$
\int_{\Omega} \frac{1}{2}\left((\Delta u)^{2}+\frac{1-\sigma}{2}\left(u_{12}^{2}-u_{11} u_{22}\right)\right) d x \geq C\|u\|^{2}, \forall u \in H^{2}(\Omega) \cap H_{0}^{1}(\Omega) .
$$

Por outro lado, $-\sigma\left(u_{11}^{2}+u_{22}^{2}\right) \geq 2 \sigma u_{11} u_{22}$, logo

$$
\begin{aligned}
\frac{1}{2}(\Delta u)^{2}+\frac{1-\sigma}{2}\left(u_{12}^{2}-u_{11} u_{22}\right) & \leq \frac{1}{2}\left(u_{11}^{2}+u_{22}^{2}\right)+\frac{1-\sigma}{2} u_{12}^{2}-\frac{\sigma}{4}\left(u_{11}^{2}+u_{22}^{2}\right) \\
& =\frac{2-\sigma}{4}\left(u_{11}^{2}+u_{22}^{2}\right)+\frac{1-\sigma}{2} u_{12}^{2} .
\end{aligned}
$$

Esta desigualdade nos diz que existe $c>0$, tal que

$$
\int_{\Omega}\left(\frac{1}{2}(\Delta u)^{2}+\frac{1-\sigma}{2}\left(u_{12}^{2}-u_{11} u_{22}\right)\right) d x \leq c\|u\|^{2}, \forall u \in H^{2}(\Omega) \cap H_{0}^{1}(\Omega) .
$$

Junto com (2.35) isto mostra que \|\|$_{J}$ é uma norma equivalente a \|\| em $H^{2}(\Omega) \cap$ $H_{0}^{1}(\Omega)$ também no caso $-1<\sigma<0$.

Então aplicando o Teorema C.4, temos que $J$ tem um mínimo global o qual é a solução fraca procurada. De fato, para $u \in \mathcal{C}^{2}(\bar{\Omega}) \cap \mathcal{C}_{0}^{0}(\bar{\Omega})$, temos seguindo (1.17) e (1.18),

$$
J(u)=\int_{\Omega}\left(\frac{1}{2}(\Delta u)^{2}-f u\right) d x+\int_{\partial \Omega} \frac{\sigma-1}{2} k\left(\frac{\partial u}{\partial \nu}\right)^{2} d S .
$$

Como essa igualdade vale em um subconjunto denso de $H^{2}(\Omega) \cap H_{0}^{1}(\Omega)$, u é um ponto 
crítico deste outro funcional, mas este é o funcional energia do problema

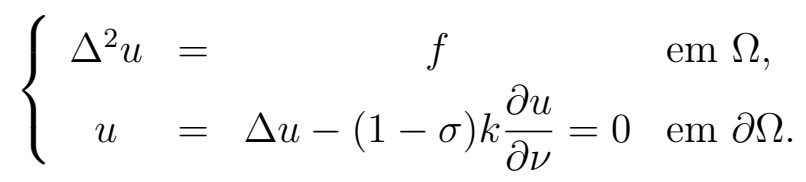

Portanto $u$ é uma solução fraca de (1.21).

Como $\Omega$ é convexo, $k \geq 0, k \neq 0$ e $\partial \Omega$ é $\mathcal{C}^{2}$, a curvatura $k$ é também contínua em $\partial \Omega$. Podemos tomar $k$ no lugar da função $b$ no Teorema 2.15. Provamos que existe solução para $\sigma \in(-1,1)$, logo necessariamente $\delta_{1, k} \geq 2$, e

$$
(1-\sigma) k \leq \delta_{1, k} k, \quad(1-\sigma) k \neq \delta_{1, k} k
$$

Se $f \geq 0$, como $\sigma<1$ e $\delta_{c, k}<0$, então $0<1-\sigma$. Temos assim $\delta_{c, k} k<(1-\sigma) k$, aplicando o Teorema 2.15 , item $3 a$, obtemos que $u \geq 0$.

\subsection{Problemas de autovalor}

Sejam $\Omega \subset \mathbb{R}^{N}$ um aberto limitado com fronteira de classe $\mathcal{C}^{2}$ e $\lambda$ um número real. Dizemos que $\lambda$ é um autovalor de $\Delta^{2}$ em $\Omega$, sob condição de Dirichlet ou de Navier, se existe $u \in \mathcal{H} \backslash\{0\}$, chamada de autofunção, solução fraca do problema (2.37)

$$
\left\{\begin{aligned}
\Delta^{2} u & =\lambda u, \quad \text { em } \Omega, \quad u \neq \equiv 0 \\
\mathcal{B} u & =0 \quad \text { em } \partial \Omega
\end{aligned}\right.
$$

onde $\mathcal{H}=H_{0}^{2}(\Omega)$ quando $\mathcal{B}$ é a condição de Dirichlet e $\mathcal{H}=H^{2}(\Omega) \cap H_{0}^{1}(\Omega)$ quando $\mathcal{B}$ é a condição de Navier.

Uma solução fraca de (2.37) é uma $u \in \mathcal{H}$, tal que

$$
\int_{\Omega} \Delta u \Delta v d x=\int_{\Omega} \lambda u v d x, \quad \forall v \in \mathcal{H}
$$

Se $\lambda \in \mathbb{R}$ é um autovalor de $\Delta^{2}$ em $\Omega$, então existe $u_{\lambda} \in \mathcal{H} \backslash\{0\}$ que é uma autofunção correspondente. Em particular, temos

$$
\int_{\Omega}\left(\Delta u_{\lambda}\right)^{2} d x=\lambda \int_{\Omega} u_{\lambda}^{2} d x
$$

e portanto $\lambda \geq 0$. Na verdade todos os autovalores são positivos. Por contradição suponha 
que 0 seja um autovalor. Seja $u_{0} \in \mathcal{H}$ uma autofunção associada a 0 . Assim $u_{0}$ é uma solução fraca não-trivial do problema

$$
\left\{\begin{array}{ccc}
\Delta^{2} u & =0 & \text { em } \Omega, \\
\mathcal{B} u & =0 & \text { em } \partial \Omega .
\end{array}\right.
$$

Mas tal solução não existe em virtude do Teorema 2.4. De outra forma, em (2.38) teríamos

$$
\int_{\Omega}\left(\Delta u_{0}\right)^{2} d x=0
$$

isto é, $\left\|u_{0}\right\|=0$, o que contradiz $u_{0} \neq 0$.

O menor autovalor de $\Delta^{2}$ sob condição de Dirichlet ou de Navier pode ser caracterizado como

$$
\lambda_{1}=\inf _{u \in \mathcal{H} \backslash\{0\}} \frac{\int_{\Omega} \Delta u^{2} d x}{\int_{\Omega} u^{2} d x} .
$$

Note que assim definido $\lambda_{1} \geq 0$. Além disso, se $\lambda \in \mathbb{R}$ é um autovalor com autofunção $u_{\lambda} \in \mathcal{H} \backslash\{0\}$, temos

$$
\lambda=\frac{\int_{\Omega} \Delta\left(u_{\lambda}\right)^{2} d x}{\int_{\Omega}\left(u_{\lambda}\right)^{2} d x} \geq \inf _{u \in \mathcal{H} \backslash\{0\}} \frac{\int_{\Omega}(\Delta u)^{2} d x}{\int_{\Omega} u^{2} d x}=\lambda_{1},
$$

ou seja, $\lambda_{1}$ definido acima é menor que ou igual a qualquer autovalor. A seguir mostramos que existe uma sequência $\left(\lambda_{n}\right)$ de autovalores de $\Delta^{2}$, cujo primeiro termo é $\lambda_{1}$ definido acima. Em seguida, provamos que a autofunção associada a $\lambda_{1}$ é positiva quando o problema linear correspondente preserva positividade.

Teorema 2.18. Seja $\Omega \subset \mathbb{R}^{N}$ um aberto, limitado com fronteira de classe $\mathcal{C}^{2}$. Os autovalores de $\Delta^{2}$ sob condição de contorno $\mathcal{B}$ formam uma sequência $\left(\lambda_{n}\right)$ não-decrescente ilimitada, tal que $\lambda_{1}$ é definido por (2.40). Além disso, existe umabase ortonormal de $\mathcal{H}$ formada por autofunções associadas a $\left(\lambda_{n}\right)$, isto é, existe uma sequência $\left(u_{n}\right) \in \mathcal{H} \backslash\{0\}$, que forma uma base para $\mathcal{H}$, tal que

$$
\begin{gathered}
\left(u_{i}, u_{j}\right)=\int_{\Omega} \Delta u_{i} \Delta u_{j} d x=\delta_{i j}, \quad \forall i, j \in \mathbb{N}, \quad e \\
\int_{\Omega} \Delta u_{i} \Delta u d x=\lambda_{i} \int_{\Omega} u_{i} u d x, \quad \forall i \in \mathbb{N}, \forall u \in \mathcal{H} .
\end{gathered}
$$


Demonstração. Seja $Q: \mathcal{H} \backslash\{0\} \longrightarrow \mathbb{R}$, definido por

$$
Q(u)=\frac{\int_{\Omega}(\Delta u)^{2} d x}{\int_{\Omega} u^{2} d x}
$$

e veja que $\lambda_{1}=\inf _{u \in \mathcal{H} \backslash\{0\}} Q(u)$. Além disso, dada $u \in \mathcal{H} \backslash\{0\}$, para qualquer $t \in \mathbb{R} \backslash\{0\}$ temos $Q(t u)=Q(u)$, e assim

$$
\lambda_{1}=\inf _{u \in \mathcal{H},|u|_{2}=1} \int_{\Omega}(\Delta u)^{2} d x
$$

O funcional $Q$ é limitado inferiormente por 0 . Tome uma sequência minimizante de $Q$, tal que $\left(v_{k}\right) \in \mathcal{H},\left\|v_{k}\right\|_{2}=1$ e $Q\left(v_{k}\right) \longrightarrow \lambda_{1}$. Como $\left\|v_{k}\right\|_{2}=1$ para todo $k$, a terceira condição nos diz que $\left\|v_{k}\right\|^{2} \longrightarrow \lambda_{1}$. Em particular, $\left(v_{k}\right)$ é uma sequência limitada em $\mathcal{H}$. Como $\mathcal{H}$ é reflexivo, existe uma subsequência de $\left(v_{k}\right)$, ainda denotada $\left(v_{k}\right)$, e uma certa $w_{1} \in \mathcal{H}$ tais que $v_{k} \rightarrow w_{1}$ em $\mathcal{H}$. Pelo Teorema A.15, existe uma subsequência de $\left(v_{k}\right)$ que converge em $L^{2}(\Omega)$ e portanto

$$
\int_{\Omega} v_{k}^{2} d x \longrightarrow \int_{\Omega} w_{1}^{2} d x
$$

Como $\int_{\Omega} v_{k}^{2} d x=1$ para todo $k$, temos $\int_{\Omega} w_{1}^{2} d x=1$ e $w_{1} \neq 0$

Por fim, usando a semicontinuidade inferior por sequências fracamente convergentes da norma de $\mathcal{H}$, obtemos

$Q\left(w_{1}\right)=\frac{\int_{\Omega}\left(\Delta w_{1}\right)^{2} d x}{\int_{\Omega} w_{1}^{2} d x}=\int_{\Omega}\left(\Delta w_{1}\right)^{2} d x \leq \liminf _{k \rightarrow \infty} \int_{\Omega}\left(\Delta v_{k}\right)^{2} d x=\liminf _{k \rightarrow \infty} \frac{\int_{\Omega}\left(\Delta v_{k}\right)^{2} d x}{\int_{\Omega}\left(v_{k}^{2}\right) d x}=\lambda_{1}$.

Como $w_{1} \in \mathcal{H} \backslash\{0\}$, segue $Q\left(w_{1}\right)=\lambda_{1}$. Conforme observamos anteriormente, tomando $u_{1}=w_{1} /\left\|w_{1}\right\|$, temos ainda $Q\left(u_{1}\right)=\lambda_{1}$.

Para verificar que $u_{1}$ é uma autofunção associada a $\lambda_{1}$, vamos olhar para o funcional $J_{1}: \mathcal{H} \longrightarrow \mathbb{R}$, definido por

$$
J_{1}(u)=\int_{\Omega}\left(\frac{(\Delta u)^{2}}{2}-\lambda_{1} \frac{u^{2}}{2}\right) d x
$$


Este funcional é de classe $\mathcal{C}^{2}(\mathcal{H})$ e para $u, v \in \mathcal{H}$

$$
J_{1}^{\prime}(u) v=\int_{\Omega} \Delta u \Delta v d x-\lambda_{1} \int_{\Omega} u v d x
$$

de modo que pontos críticos de $J_{1}$ são autofunções associadas a $\lambda_{1}$. Pela definição de $\lambda_{1}$, temos

$$
\int_{\Omega}(\Delta u)^{2} d x \geq \lambda_{1} \int_{\Omega} u^{2} d x, \quad \forall u \in \mathcal{H}
$$

Assim $J_{1}(u) \geq 0$ para toda $u$ em $\mathcal{H}$. Esta observação nos diz que $u_{1}$ é um ponto mínimo global para $J_{1}$, pois

$$
J_{1}\left(u_{1}\right)=\frac{1}{2}\left(\int_{\Omega}\left(\left(\Delta u_{1}\right)^{2}-\lambda_{1} u_{1}^{2}\right) d x\right)=0 .
$$

Pela Proposição C.1, $u_{1}$ é um ponto crítico de $J_{1}$ e, portanto, uma autofunção associada a $\lambda_{1}$.

Suponha por indução que dado $n \in \mathbb{N}$, existem $\lambda_{1} \leq \ldots \leq \lambda_{n}$ e $u_{1}, \ldots, u_{n} \in \mathcal{H} \backslash\{0\}$ tais que

$$
\begin{gathered}
\int_{\Omega} \Delta u_{i} \Delta u_{j} d x=\delta_{i j}, \quad \forall i, j \in\{1, \ldots, n\}, \\
\int_{\Omega} \Delta u_{i} \Delta u d x=\lambda_{i} \int_{\Omega} u_{i} u d x, \quad \forall i \in\{1, \ldots, n\}, \forall u \in \mathcal{H} .
\end{gathered}
$$

Defina $V_{n}=\left\{u \in \mathcal{H} ; \int_{\Omega} \Delta u \Delta u_{i} d x=0, \forall i \in\{1, \ldots, n\}\right\}$, note que $V_{n}$ é um subespaço fechado de $\mathcal{H}$. Tome

$$
\lambda_{n+1}=\inf _{u \in V_{n} \backslash\{0\}} Q(u) .
$$

Observando que $V_{n+1} \subset V_{n}$, temos $\lambda_{n} \leq \lambda_{n+1}$.

Argumentando como acima, existe $u_{n+1} \in V_{n} \backslash\{0\}$ tal que $\lambda_{n+1}=Q\left(u_{n+1}\right)$. Além disso, $u_{n+1}$ é um ponto critico do funcional $J_{n+1}: V_{n} \longrightarrow \mathbb{R}$, definido por $J_{n}(u)=$ $\int_{\Omega}\left(\frac{(\Delta u)^{2}}{2}-\lambda_{n+1} \frac{u^{2}}{2}\right) d x$, isto é,

$$
\int_{\Omega} \Delta u_{n+1} \Delta u d x=\lambda_{n+1} \int_{\Omega} u_{n+1} u d x, \quad \forall u \in V_{n}
$$

Por outro lado, para $u \in \mathcal{H} \backslash V_{n}$, observe que $u=\sum_{i=1}^{n} s_{i} u_{i}$. Desta forma, pela 
construção de $V_{n}$,

$$
\begin{aligned}
\int_{\Omega} u_{n+1} u d x & =\int_{\Omega} u_{n+1}\left(\sum_{i=1}^{n} s_{i} u_{i}\right) d x=\sum_{i=1}^{n} s_{i} \int_{\Omega} u_{n+1} u_{i} d x \\
& =\sum_{i=1}^{n} s_{i} \frac{1}{\lambda_{i}} \int_{\Omega} \Delta u_{n+1} \Delta u_{i} d x=0=\lambda_{n+1} \int_{\Omega} \Delta u_{n+1} \Delta u d x
\end{aligned}
$$

Assim $u_{n+1}$ é uma autofunção associada a $\lambda_{n+1}$.

Pelo princípio de indução finita, existe uma sequência não-decrescente $\left(\lambda_{n}\right)$ de autovalores de $\Delta^{2}$ sob condição de contorno $\mathcal{B}$ e as autofunções associadas cumprem

$$
\int_{\Omega} \Delta u_{i} \Delta u_{j} d x=\delta_{i j}, \quad \forall i, j \in \mathbb{N}
$$

Resta verificar que $\left(\lambda_{n}\right)$ é ilimitada, o que faremos argumentando por contradição. Suponha que $\left(\lambda_{n}\right)$ é limitada, como a sequência é monótona, existe $\lambda_{0}$ tal que $\lambda_{n} \longrightarrow \lambda_{0}$. As autofunções $u_{n}$ cumprem $\left\|u_{n}\right\|^{2}=Q\left(u_{n}\right)=\lambda_{n}$, logo $\left(u_{n}\right)$ é uma sequência limitada em $\mathcal{H}$, e portanto possui uma subsequência convergente em $L^{2}(\Omega)$. Mas isto é uma contradição, pois por ortogonalidade

$$
\left\|u_{k}-u_{j}\right\|_{2}^{2}=\left\|u_{k}\right\|_{2}^{2}+\left\|u_{j}\right\|_{2}^{2}=2
$$

para quaisquer naturais $j \neq k$.

Para verificar que $\left(u_{n}\right)$ é uma base, suponha por contradição que existe $u \neq 0$ tal que $u \in \bigcap_{n=1}^{\infty} V_{n}$. Como $Q(u)=\lambda \in \mathbb{R}$ e $\left(\lambda_{n}\right)$ é ilimitada, podemos tomar $n_{0} \in \mathbb{N}$ tal que $\lambda_{n_{0}}>\lambda$. Temos então

$$
\lambda=Q(u) \geq \inf _{v \in V_{n_{0}}} Q(v)=\lambda_{n_{0}}>\lambda,
$$

mas isto é uma contradição.

Portanto $u \in \mathcal{H},\left(u, u_{n}\right)=0$, para todo $n \in \mathbb{N}$ implica $u=0$, e assim $\left(u_{n}\right)$ é uma base ortonormal de $\mathcal{H}$.

Observação 2.19. As autofunções associadas ao operador $\Delta^{2}$ no domínio $\Omega$ são as mesmas do operador Laplaciano somente quando considerada condição de contorno de Navier. Para condição de contorno de Dirichlet ou Steklov, as autofunções são diferentes.

A seguir mostramos que a autofunção associada a $\lambda_{1}$ tem sinal definido quando o problema linear correspondente preserva positividade. 
Vamos empregar o método de decomposição em cones duais para obter a positividade da primeira autofunção quando o operador $\Delta^{2}$ sob a condição de contorno $\mathcal{B}$ preserva positividade. Para isto, introduzimos algumas definições que também serão exploradas em outros pontos.

Um cone em um espaço de Hilbert $\mathcal{H}$ é um conjunto $K \subset \mathcal{H}$ tal que $u \in K, t \geq 0$ implica $t u \in K$. O cone dual de $K$, denotado aqui por $K^{*}$, é o conjunto

$$
K^{*}=\{v \in \mathcal{H} ;(u, v) \leq 0, \forall u \in K\}
$$

Quando $K$ é um cone convexo fechado e não-vazio chamamos $K$ e $K^{*}$ de cones duais.

Vamos usar a seguinte proposição de Moreau [18, Proposition 1].

Proposição 2.20. Sejam $\mathcal{H}$ um espaço de Hilbert e $P, Q \subset \mathcal{H}$ dois cones mutualmente duais. Então $\mathcal{H}$ pode ser decomposto em $P \oplus Q$, isto é, para cada $z \in \mathcal{H}$ existe um único $\operatorname{par}(x, y) \in P \times Q$ tal que $z=x+y e(x, y)=0$. Além disso, o par $(x, y)$ é caracterizado por $x=\operatorname{proj}_{P} z, y=\operatorname{proj}_{Q} z$.

Corolário 2.21. Sejam $\mathcal{H} \in\left\{H_{0}^{2}(\Omega), H^{2}(\Omega) \cap H_{0}^{1}(\Omega)\right\}, \Omega \subset \mathbb{R}^{N}$ um aberto limitado com fronteira de classe $\mathcal{C}^{2} e$

$$
K=\{u \in \mathcal{H} ; u(x) \geq 0 \text { em quase todo ponto } x \in \Omega\}
$$

Para toda $u \in \mathcal{H}$, existem únicas $v \in K, w \in K^{*}$ tais que

$$
u=v+w, \quad(v, w)=0 .
$$

Demonstração. Basta verificar que $K$ é um cone convexo e fechado. Claramente, pela definição de $K$, temos que $K$ é um cone. Sejam $u, v \in K$ e $t \in[0,1]$. Se para $x \in \Omega$, $u(x) \geq 0, v(x) \geq 0$, temos

$$
t u(x)+(1-t) v(x) \geq \min \{u(x), v(x)\} \geq 0 .
$$

Como $\{x \in \Omega ; u(x)<0$ ou $v(x)<0\}$ tem medida nula, segue $t u+(1-t) v \in K$, e $K$ é convexo.

Para verificar que $K$ é fechado, tome $\left(u_{k}\right) \in K$, tal que $u_{k} \longrightarrow u \in \mathcal{H}$. Pela imersão de $\mathcal{H}$ em $L^{2}(\Omega)$, temos $u_{k} \longrightarrow u$ em $L^{2}(\Omega)$. Então existe uma subsequência $\left(u_{k_{n}}\right)$ tal que $u_{k_{n}}(x) \longrightarrow u(x)$ para quase todo $x \in \Omega$. 
Agora observe que

$$
\left|\bigcup_{n \geq 1} u_{k_{n}}^{-1}((-\infty, 0))\right| \leq \sum_{n=1}^{\infty}\left|u_{k_{n}}^{-1}((-\infty, 0))\right|=0
$$

Assim, para qualquer $x \in \Omega$ tal que $u_{k_{n}}(x) \geq 0$ para todo $n \geq 1$ e $u_{k_{n}}(x) \longrightarrow u(x)$ temos que $u(x) \geq 0$. Logo, a menos de um conjunto de medida nula, $u^{-1}((-\infty, 0)) \subset$ $\bigcup_{k \geq 1} u_{k}^{-1}((-\infty, 0))$. Portanto, $u \geq 0$ em quase todo ponto de $\Omega$, isto é, $u \in K$.

Para empregar esta decomposição vamos precisar do seguinte princípio de comparação, o qual nos diz que o cone dual do cone $K$ definido em (2.41) está contido em $-K$.

Proposição 2.22. Seja $\Omega \subset \mathbb{R}^{N}$ um aberto limitado com fronteira de classe $\mathcal{C}^{2}$ e $K$ o cone definido em (2.41). Se $\Delta^{2}$ sob a condição de contorno $\mathcal{B}$ preserva positividade em $\Omega$ e $v \in K^{*}$, então $v \leq 0$ em quase todo ponto de $\Omega$.

Demonstração. Sejam $\varphi \in \mathcal{C}_{c}^{\infty}(\Omega), \varphi \geq 0$ e $u_{\varphi} \in \mathcal{H}$ a solução de

$$
\left\{\begin{array}{cccc}
\Delta^{2} u_{\varphi} & =\varphi & \text { em } & \Omega \\
\mathcal{B} u_{\varphi} & =0 & \text { em } & \partial \Omega
\end{array}\right.
$$

Pela hipótese de preservação de positividade $u_{\varphi} \geq 0$ em $\Omega$, $\operatorname{logo} u_{\varphi} \in K$. Como $v \in K^{*}$, temos

$$
\int_{\Omega} v \varphi d x=\int_{\Omega} \Delta u_{\varphi} \Delta v d x \leq 0, \quad \forall \varphi \in \mathcal{C}_{c}^{\infty}(\Omega) \cap K
$$

e $\operatorname{assim} v \leq 0$ em $\Omega$.

Teorema 2.23. Se $\Omega \subset \mathbb{R}^{N}$ é um aberto com fronteira de classe $\mathcal{C}^{2}$ e $\Delta^{2}$ sob a condição de contorno $\mathcal{B}$ preserva positividade, então qualquer autofunção associada a $\lambda_{1}$ não muda de sinal em $\Omega$. Além disso, $\lambda_{1}$ é simples.

Demonstração. Sejam $\lambda_{1}$ o primeiro autovalor de $\Delta^{2}$ definido em $(2.40)$ e $u_{1} \in \mathcal{H}$ uma autofunção associada. Pelo Teorema 2.18, $u_{1} \neq 0$. Decompondo $u_{1}$ como na Proposição 2.20 podemos escrever

$$
u_{1}=v_{1}+w_{1}, \quad v_{1} \in K, w_{1} \in K^{*} .
$$

Além disso, segundo a Proposição $2.22, K^{*} \subset-K$ e portanto $w_{1} \leq 0 \mathrm{em} \Omega$.

Por contradição suponha que $u_{1}$ mude de sinal. Assim $v_{1} \neq 0$ e $w_{1} \neq 0$. Defina então $\widetilde{u}_{1}=v_{1}-w_{1}$ e observe que $\widetilde{u}_{1} \in K$. Além disso, $\widetilde{u}_{1} \geq\left|u_{1}\right|$ em $\Omega$ e $\widetilde{u}_{1}>\left|u_{1}\right|$ em um 
conjunto de medida positiva e portanto

$$
\int_{\Omega}\left|\widetilde{u}_{1}\right|^{2} d x>\int_{\Omega}\left|u_{1}\right|^{2} d x
$$

Por outro lado,

$$
\left\|\widetilde{u}_{1}\right\|^{2}=\left\|v_{1}-w_{1}\right\|^{2}=\left\|v_{1}\right\|^{2}+\left\|w_{1}\right\|^{2}=\left\|v_{1}+w_{1}\right\|^{2}=\left\|u_{1}\right\|^{2}
$$

pois $v_{1}$ e $w_{1}$ são ortogonais. Dessa forma,

$$
Q\left(\widetilde{u}_{1}\right)=\frac{\int_{\Omega} \Delta \widetilde{u}_{1}^{2} d x}{\int_{\Omega} \widetilde{u}_{1}^{2} d x}<\frac{\int_{\Omega} \Delta \widetilde{u}_{1}^{2} d x}{\int_{\Omega} u_{1}^{2} d x}=\frac{\int_{\Omega} \Delta u_{1}^{2} d x}{\int_{\Omega} u_{1}^{2} d x}=\lambda_{1}
$$

o que contradiz a definição de $\lambda_{1}$. Portanto, $u_{1}$ não pode mudar de sinal em $\Omega$.

Por contradição suponha a existência de autofunções $u$ e $v$ associadas a $\lambda_{1}$ linearmente independentes, podemos assumir que $u$ e $v$ são não-negativas. Para cada $m \in \mathbb{R}, u_{m}=$ $m u+v$ ainda é uma autofunção associada a $\lambda_{1}$.

Tome $s \in \mathbb{R}$ tal que

$$
\int_{\Omega} u u_{s} d x=0, \quad \text { isto é, } \quad s=-\frac{\int_{\Omega} u v d x}{\int_{\Omega} u^{2} d x} .
$$

Como $u_{s}$ também não muda de sinal, concluímos que $u(x) u_{s}(x)=0$ em quase todo ponto $x$ de $\Omega$. Se $u_{s}=0$, então temos uma contradição pois $u$ e $v$ são linearmente independentes. Por outro lado, se $u_{s}$ é diferente de 0 , então $u^{-1}(\mathbb{R} \backslash\{0\}) \cap u_{s}^{-1}(\mathbb{R} \backslash\{0\})$ tem medida nula, enquanto que cada um dos conjuntos tem medida positiva. Se $u_{s} \geq 0$, então $z=u-u_{s}$ é uma autofunção que muda de sinal, uma contradição. Se $u_{s} \leq 0$, então $z=u+u_{s}$ é uma autofunção que muda de sinal.

Portanto $\lambda_{1}$ é um autovalor simples.

Teorema 2.24. Sejam $\Omega \subset \mathbb{R}^{N}$ um aberto limitado com fronteira de classe $\mathcal{C}^{2}$. Se $\Delta^{2}$ preserva positividade em $\Omega$ sob condição de Dirichlet, $\lambda_{1}$ é o primeiro autovalor de $\Delta^{2}$ sob condição de Navier e $\Lambda_{1}$ é o primeiro autovalor sob condição de Dirichlet, então $\lambda_{1}<\Lambda_{1}$.

Demonstração. De fato, como $H_{0}^{2}(\Omega) \subset H^{2}(\Omega) \cap H_{0}^{1}(\Omega)$, temos $\lambda_{1} \leq \Lambda_{1}$. Para mostrar a desigualdade estrita basta verificar que $u_{1} \notin H_{0}^{2}(\Omega)$. 
Reescrevendo (2.37) como um sistema, temos

$$
\left\{\begin{array}{rlll}
-\Delta u_{1}= & v & \text { em } \Omega \\
-\Delta v & =\lambda_{1} u_{1} & & \text { em } \Omega \\
u=v & = & & \text { em } \partial \Omega
\end{array}\right.
$$

Como $u_{1} \geq 0$ em $\Omega$ e $\lambda_{1}>0$, o Corolário A.19 implica $v \geq 0$ em $\Omega$. Como $u_{1} \in H^{2}(\Omega)$, temos $-\Delta\left(u_{1}\right) \geq 0$ em $\Omega$. Pelo Lema de Hopf, $\frac{\partial u_{1}}{\partial \nu}<0$ em $\partial \Omega$, $\log \mathrm{O}$

$$
u_{1} \notin H_{0}^{2}(\Omega)
$$

Em particular $u_{1} \neq \widetilde{u}_{1}$, onde $\widetilde{u}_{1}$ é a autofunção associada a $\Lambda_{1}$. Além disso,

$$
\lambda_{1}=\frac{\int_{\Omega} \Delta u_{1}^{2} d x}{\int_{\Omega} u_{1}^{2} d x}<\frac{\int_{\Omega} \Delta \widetilde{u}_{1}^{2} d x}{\int_{\Omega} \widetilde{u}_{1}^{2} d x}=\Lambda_{1} .
$$

Em vista da discussão deste capítulo, o Teorema 2.23 é válido em um domínio $\Omega \subset \mathbb{R}^{N}$ qualquer com fronteira de classe $\mathcal{C}^{2}$, quando consideramos a condição de contorno de Navier. Por outro lado, para a condição de contorno de Dirichlet o Teorema 2.23 só é válido para alguns casos, como por exemplo a bola unitária de $\mathbb{R}^{N}$ ou os limaçons $\Omega_{a}$ com $a \in[0, \sqrt{6} / 6]$.

Para a condição de Steklov, um autovalor é um $\lambda \in \mathbb{R}$ tal que existe uma solução fraca não-trivial de

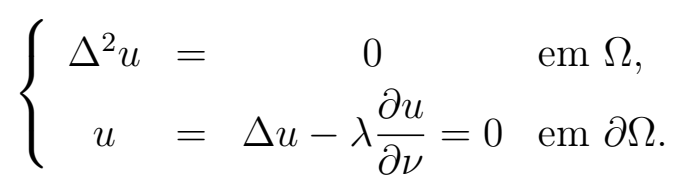

Isto significa que $u \in H^{2}(\Omega) \cap H_{0}^{1}(\Omega) \backslash\{0\}$ e $\int_{\Omega} \Delta u \Delta v d x=\lambda \int_{\partial \Omega} \frac{\partial u}{\partial \nu} \frac{\partial v}{\partial \nu} d S$, para qualquer função $v \in H^{2}(\Omega) \cap H_{0}^{1}(\Omega)$.

Vamos definir um primeiro autovalor com peso para o problema de Steklov, que foi empregado na Seção 2.4 .

Teorema 2.25. Sejam $\Omega \subset \mathbb{R}^{N}$ um aberto limitado com fronteira $\mathcal{C}^{2}, N \geq 2, b: \partial \Omega \longrightarrow \mathbb{R}$ continua não-negativa, não-nula,

$$
\delta_{1, b}=\inf _{u \in P} \frac{\int_{\Omega} \Delta u^{2} d x}{\int_{\partial \Omega} b\left(\frac{\partial u}{\partial \nu}\right)^{2} d S}
$$


e $P=\left\{u \in H^{2}(\Omega) \cap H_{0}^{1}(\Omega) ; \int_{\partial \Omega} b\left(\frac{\partial u}{\partial \nu}\right)^{2} d S \neq 0\right\}$. Existe $u_{1, b} \in P$ tal que

$$
\int_{\Omega}\left(\Delta u_{1, b}\right)^{2} d x=\delta_{1, b} \int_{\partial \Omega} b\left(\frac{\partial u_{1, b}}{\partial \nu}\right)^{2} d S .
$$

Além disso, $u_{1, b}$ é positiva em $\Omega,-\Delta u_{1, b}>0$ em $\Omega$ e $\frac{\partial u_{1, b}}{\partial \nu}<0$ em $\partial \Omega$. A menos de múltiplos, $u_{1, b}$ é única.

Demonstração. Seja $b: \partial \Omega \longrightarrow \mathbb{R}$ não-negativa, não-nula e considere o funcional $J_{b}$ : $H^{2}(\Omega) \cap H_{0}^{1}(\Omega) \backslash\{0\} \longrightarrow \mathbb{R} \cup\{\infty\}$, definido por

$$
J_{b}(u)=\frac{\int_{\Omega} \Delta u^{2} d x}{\int_{\partial \Omega} b\left(\frac{\partial u}{\partial \nu}\right)^{2} d S}, \text { se } \int_{\partial \Omega} b\left(\frac{\partial u}{\partial \nu}\right)^{2} d S \neq 0
$$

e

$$
J_{b}(u)=\infty, \text { se } \int_{\partial \Omega} b\left(\frac{\partial u}{\partial \nu}\right)^{2} d S=0 .
$$

$J_{b}$ é positivo e assim podemos tomar

$$
\delta_{1, b}=\inf _{u \in P} J_{b}(u) \geq 0
$$

Vamos verificar que este ínfimo é atingido, e assim $\delta_{1, b}>0$.

Observamos que $J_{b}(\lambda u)=J_{b}(u)$, para todo $\lambda \neq 0$ e para toda $u \neq 0$, logo $J_{b}\left(H^{2}(\Omega) \cap\right.$ $\left.H_{0}^{1}(\Omega)\right)=J_{b}\left(\left\{u \in H^{2}(\Omega) \cap H_{0}^{1}(\Omega) ;\|u\|=1\right\}\right)$. Logo $\delta_{1, b}=\inf _{B_{H}} J_{b}(u)$, onde $B_{H}=\{u \in$ $\left.H^{2}(\Omega) \cap H_{0}^{1},\|u\| \leq 1\right\}$.

Seja $\left(u_{k}\right) \in B_{H}$ tal que $J_{b}\left(u_{k}\right) \longrightarrow \delta_{1, b}$. Como $J_{b}(u)=\infty$ para $\int_{\partial \Omega} b\left(\frac{\partial u}{\partial \nu}\right)^{2} d S=0$, podemos assumir que $\int_{\partial \Omega} b\left(\frac{\partial u_{k}}{\partial \nu}\right)^{2} d S \neq 0$ para todo $k$.

Como $B_{H}$ é compacto na topologia fraca, existem $u \in B_{H}$ e uma subsequência de $\left(u_{k}\right)$, ainda denotada $\left(u_{k}\right)$, tais que $u_{k} \rightarrow u$.

Vamos provar que $J_{b}$ é semicontínuo inferiormente por sequências em todos os pontos $u \in B_{H}$ tais que $\int_{\partial \Omega} b\left(\frac{\partial u}{\partial \nu}\right)^{2} d S \neq 0$.

Seja $u_{k} \rightarrow u \in B_{H}$, pela compacidade do traço existe uma subsequência $\left(u_{k_{j}}\right)$ tal que

$$
\int_{\partial \Omega} b\left(\frac{\partial u}{\partial \nu}\right)^{2} d S=\lim _{j \rightarrow \infty} \int_{\partial \Omega} b\left(\frac{\partial u_{k_{j}}}{\partial \nu}\right)^{2} d S
$$


Assim

$$
\liminf \int_{\partial \Omega} b\left(\frac{\partial u_{k}}{\partial \nu}\right)^{2} d S \leq \liminf \int_{\partial \Omega} b\left(\frac{\partial u_{k_{j}}}{\partial \nu}\right)^{2} d S=\int_{\partial \Omega} b\left(\frac{\partial u}{\partial \nu}\right)^{2} d S
$$

e como $\int_{\partial \Omega} b\left(\frac{\partial u}{\partial \nu}\right)^{2} d S \neq 0$

$\liminf J_{b}\left(u_{k}\right)=\liminf \left(\int_{\partial \Omega} b\left(\frac{\partial u_{k}}{\partial \nu}\right)^{2} d S\right)^{-1} \geq\left(\int_{\partial \Omega} b\left(\frac{\partial u}{\partial \nu}\right)^{2} d S\right)^{-1} \geq J(u)$

Por (2.46), chegamos a

$$
\delta_{1, b}=\lim J_{b}\left(u_{k}\right)=\liminf J_{b}\left(u_{k}\right) \geq J_{b}(u),
$$

$\operatorname{assim} J_{b}(u)=\delta_{1, b}$, e $u$ minimiza $J_{b}$. É claro que $u \neq 0$, e $\operatorname{assim} \delta_{1, b}=J_{b}(u)>0$.

Para verificar que $u$ é autovalor, basta observar como na prova do Teorema 2.18 que $u$ é ponto mínimo global de

$$
J(u)=\frac{1}{2} \int_{\Omega}(\Delta u)^{2} d x-\frac{1}{2} \int_{\partial \Omega} b\left(\frac{\partial u}{\partial \nu}\right)^{2} d S .
$$

Para provar as outras propriedades de $u=u_{1, b}$, seja $\widetilde{u} \in H^{2}(\Omega) \cap H_{0}^{1}(\Omega)$ a única solução de $-\Delta \widetilde{u}=\left|\Delta u_{1, b}\right|$, com $u=0$ na fronteira de $\Omega$. Temos então $-\Delta \widetilde{u} \geq \pm \Delta u_{1, b}$, e pelo princípio do máximo $-\Delta\left(\widetilde{u} \pm u_{1, b}\right) \geq 0, \widetilde{u} \pm u_{1, b} \in H_{0}^{1}(\Omega)$ implica $\widetilde{u} \geq\left|u_{1, b}\right|$ em $\Omega$.

Além disso, $\frac{\partial \widetilde{u}}{\partial \nu} \leq-\left|\frac{\partial u_{1, b}}{\partial \nu}\right|$ em $\partial \Omega$. Se $\Delta u_{1, b}$ muda de sinal, segue que as desigualdades são estritas, em particular da última temos $J_{b}(\widetilde{u})<J_{b}\left(u_{1, b}\right)$, contradizendo a escolha de $u_{1, b}$. Assim, $\Delta u_{1, b}$ não troca de sinal, e a menos de trocar $u_{1, b}$ por $-u_{1, b}$, podemos assumir $-\Delta u_{1, b} \geq 0$, que nos dá $\frac{\partial u_{1, b}}{\partial \nu}<0$ em $\partial \Omega$, ou

$$
-\Delta u_{1, b}=\delta_{1, b} b \frac{\partial u_{1, b}}{\partial \nu}>0 \text { em } \partial \Omega
$$

Como $-\Delta\left(-\Delta u_{1, b}\right)=0$, pelo princípio do máximo $-\Delta u_{1, b}>0$ em $\bar{\Omega}$, assim pelo princípio do máximo forte $u_{1, b}>0 \mathrm{em} \Omega$, pois $u_{1, b} \neq 0$ por construção.

Vamos provar a simplicidade de $\delta_{1, b}$ de modo análogo ao Teorema 2.23. Se houvessem duas autofunções linearmente independentes $u_{1, b}, v_{1, b} \in H^{2}(\Omega) \cap H_{0}^{1}(\Omega)$, teríamos alguma combinação linear destas trocando de sinal, o que leva a uma contradição. 


-

\section{Uma classe de problemas semilineares}

Neste capítulo consideramos existência e propriedades qualitativas de soluções de problemas semilineares.

O problema semilinear modelo considerado é

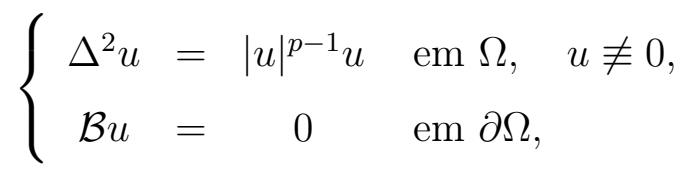

onde $p \in(0, \infty), \Omega \subset \mathbb{R}^{N}$ é um aberto limitado, $\mathcal{B}$ é uma condição de fronteira e $u \not \equiv 0$ significa que $u$ é diferente de 0 em um conjunto de medida positiva.

A potência $p$ determina a estrutura do problema. O número

$$
s=\frac{2 N}{N-4}, \quad N>4
$$

é chamado expoente crítico para a imersão de $H^{2}(\Omega)$ em $L^{p+1}(\Omega)$ no teorema de imersão A.15. Para valores de $p$ tais que $p+1>s$ não podemos aplicar métodos variacionais a (3.1). Este expoente também motivou a divisão deste capítulo. Na primeira seção estudaremos o que chamamos de problema subcrítico, nela são descritos resultados de existência de solução fraca para (3.1) por argumentos variacionais.

A segunda seção trata do problema supercrítico em domínios estrelados. Esta hipótese permite mostrar que não existem soluções clássicas não-triviais para (3.1) usando identidades do tipo de Pohozaev. 


\subsection{Problema subcrítico}

Uma solução clássica de (3.1) é uma $u \in \mathcal{C}^{4}(\bar{\Omega})$, satisfazendo cada uma das equações pontualmente. A razão para a condição, $u \not \equiv 0$ é que definindo $u(x)=0$ para todo $x \in \bar{\Omega}$ temos uma solução clássica de (3.1), chamada solução trivial.

Multiplicando o termo $|u|^{p-1} u$ por uma função suave $v$, que se anula na fronteira de $\Omega$, obtemos

$$
\int_{\Omega}|u|^{p-1} u v d x
$$

De acordo com o exemplo (B.20) este termo é a derivada do funcional $\mathcal{C}^{1}$

$$
K(u)=\int_{\Omega}|u|^{p+1} d x .
$$

Para que este funcional seja bem definido em $H^{2}(\Omega)$ precisamos garantir que $|u|^{p+1} \in$ $L^{1}(\Omega)$. De acordo com o teorema de imersão A.15, temos

$$
\int_{\Omega}|u|^{p+1} d x \leq C\|u\|^{p+1}<\infty
$$

quando $p+1$ cumpre as hipóteses do teorema. Isto motiva a seguinte definição.

Definição 3.1. Dizemos que o problema (3.1) é subcrítico se

$$
N \leq 4 \text { e } p \in(0, \infty), \quad \text { ou } \quad N>5 \text { e } p \in\left(0, \frac{N+4}{N-4}\right)
$$

Vamos definir a noção de solução fraca de acordo com a condição de fronteira considerada. Para a condição de Dirichlet,

$$
\left\{\begin{array}{c}
u=\frac{\partial u}{\partial \nu}=0 \quad \text { em } \partial \Omega \\
u \quad \not \equiv \quad
\end{array}\right.
$$

uma integração por partes de $\int_{\Omega}\left(\Delta^{2} u\right) v-|u|^{p-1} u v d x=0$ nos dá

$$
\int_{\Omega}\left(\Delta u \Delta v-|u|^{p-1} u v\right) d x=0
$$


Definição 3.2. Dizemos que $u \in H_{0}^{2}(\Omega)$ é uma solução fraca de (3.1) e (3.3) se

$$
\int_{\Omega}\left(\Delta u \Delta v-|u|^{p-1} u v\right) d x=0, \forall v \in H_{0}^{2}(\Omega)
$$

Para a condição de Navier

$$
\left\{\begin{array}{ccc}
u= & \Delta u=0 & \text { em } \partial \Omega \\
u \neq & 0 & \text { em } \Omega
\end{array}\right.
$$

temos a seguinte definição.

Definição 3.3. Uma solução fraca de (3.1) e (3.5) como uma $u \in H^{2}(\Omega) \cap H_{0}^{1}(\Omega)$, tal que

$$
\int_{\Omega}\left(\Delta u \Delta v-|u|^{p-1} u v\right) d x=0, \forall v \in H^{2}(\Omega) \cap H_{0}^{1}(\Omega) .
$$

Verifica-se que para as duas condições podemos reescrever a definição de solução fraca como

$$
J^{\prime}(u) v=0, \quad \forall v \in \mathcal{H},
$$

ou ainda $u$ é um ponto crítico do funcional $J$, onde

$$
J(u)=\int_{\Omega}\left(\frac{1}{2}(\Delta u)^{2}-\frac{1}{p+1}|u|^{p+1}\right) d x
$$

definido em $\mathcal{H}=H_{0}^{2}(\Omega)$ para a condição de Dirichlet ou $\mathcal{H}=H^{2}(\Omega) \cap H_{0}^{1}(\Omega)$ para a condição de Navier.

\subsubsection{O caso sublinear}

Considere inicialmente $0<p<1$, note que neste caso (3.1) é subcrítico em qualquer dimensão. Sob estas condições o funcional associado ao problema é coercivo e os resultados de existência são similares aos do Capítulo 2.

Seja $u$ uma solução fraca de (3.1) e (3.3), conforme observado acima $u$ é um ponto crítico do funcional $J: \mathcal{H} \longrightarrow \mathbb{R}$

$$
J(u)=\int_{\Omega}\left(\frac{1}{2}(\Delta u)^{2}-\frac{1}{p+1}|u|^{p+1}\right) d x
$$


Teorema 3.4. Se $\Omega \subset \mathbb{R}^{N}$ é um aberto limitado, então existe uma solução fraca nãotrivial para (3.1) e (3.3). Se $\partial \Omega$ é de classe $\mathcal{C}^{2}$, então existe uma solução fraca não-trivial para (3.1) e (3.5).

Demonstração. O funcional J é de classe $\mathcal{C}^{1}(\mathcal{H})$ ), vamos verificar que também é coercivo e semicontínuo inferiormente por sequências na topologia fraca. Aplicando o Teorema C.4 concluímos que $J$ assume um mínimo global. Por fim, observamos que este mínimo é atingido em um nível negativo.

De fato, observe que

$$
J(u)=\frac{1}{2}\|u\|^{2}-\frac{1}{p+1}\|u\|_{p+1}^{p+1}
$$

Usando o teorema de imersão A.15, obtemos

$$
J(u) \geq \frac{1}{2}\|u\|^{2}-c\|u\|^{p+1}=f(\|u\|)
$$

onde $f(t)=\frac{1}{2} t^{2}-c t^{p+1}$ e $c>0$. Como $\lim _{t \rightarrow \infty} f(t)=\infty, J$ é coercivo. De fato, dado $M \in \mathbb{N}$, existe $R>0$ tal que $t>R$ implica $f(t)>M$. Assim, se $u \in \mathcal{H}$ e $\|u\|>R$, temos $J(u) \geq f(\|u\|)>M$.

Precisamos agora provar a semicontinuidade inferior por sequências na topologia fraca, isto é, que se $u_{k} \rightarrow u, u_{k}, u \in \mathcal{H}$ para todo $k \in \mathbb{N}$, então $J(u) \leq \lim J\left(u_{k}\right)$. Suponha que $u_{k} \rightarrow u$, então pela semicontinuidade inferior da norma $\liminf _{k \rightarrow \infty}\left\|u_{k}\right\|^{2} \geq\|u\|^{2}$.

Como a imersão de $\mathcal{H}$ em $L^{p+1}(\Omega)$ é compacta e a sequência $\left(u_{k}\right)$ é limitada, existe uma subsequência, ainda denotada $\left(u_{k}\right)$, que é convergente em $L^{p+1}(\Omega)$. Em particular,

$$
\frac{1}{p+1} \int_{\Omega}\left|u_{k}\right|^{p+1} d x=\frac{1}{p+1}\left\|u_{k}\right\|_{p+1}^{p+1} \longrightarrow \frac{1}{p+1}\|u\|_{p+1}^{p+1}=\int_{\Omega} \frac{|u|^{p+1}}{p+1} d x
$$

Por fim, temos

$$
\begin{aligned}
\liminf _{k \rightarrow \infty} J\left(u_{k}\right) & =\liminf _{k \rightarrow \infty}\left(\frac{1}{2}\left\|u_{k}\right\|^{2}-\frac{1}{p+1}\left\|u_{k}\right\|_{p+1}^{p+1}\right) \\
& =\liminf _{k \rightarrow \infty} \frac{1}{2}\left\|u_{k}\right\|^{2}-\lim _{k \rightarrow \infty} \frac{1}{p+1}\|u\|_{p+1}^{p+1} \geq \frac{1}{2}\|u\|^{2}-\frac{1}{p+1}\|u\|_{p+1}^{p+1}=J(u) .
\end{aligned}
$$

Pelo Teorema C.4, existe $u \in \mathcal{H}$ tal que $J(u)=b$. Assim $u$ é um ponto mínimo global e, portanto, um é ponto crítico de $J$. Para ver que $u \neq 0$, vamos verificar que sempre existe $v \in \mathcal{H}$, com $J(v)<0$, $\log \mathrm{J}(u) \leq J(v)<0=J(0)$, de modo que $J(u)<J(0)$, e $u \neq 0$. 
De fato, definindo para $A, B \in(0, \infty), f(t)=A t^{2}-B t^{p+1}, f:[0, \infty) \longrightarrow \mathbb{R}$, teremos

$$
f(t)<0 \Longleftrightarrow A t^{2}<B t^{p+1} \Longleftrightarrow t^{2-p-1}<\frac{B}{A} \Longleftrightarrow t^{1-p}<\frac{B}{A}
$$

Como $0<p<1$ implica que $t^{1-p}$ é uma função contínua crescente de $t$ em $[0, \infty)$ e ainda $\lim _{t \rightarrow 0^{+}} t^{1-p}=0$, existe um intervalo no qual $t^{1-p}<\frac{B}{A}$. Escolhendo para uma $u \neq 0$, $A=\int_{\Omega} \frac{1}{2} \Delta u^{2} d x>0$ e $B=\int_{\Omega}|u|^{p+1} d x>0$, pelo argumento acima $J(t u)<0$ para um certo $t$ próximo de 0 , provando a afirmação.

Observe que, para $u \in H_{0}^{2}(\Omega) \backslash\{0\}$ e $\lambda \in \mathbb{R}$,

$$
J(\lambda u)=\int_{\Omega}\left(\frac{1}{2}(\Delta \lambda u)^{2}-\frac{1}{p+1}|\lambda u|^{p+1}\right) d x=\int_{\Omega}\left(\frac{1}{2}(\Delta u)^{2} \lambda^{2}-\frac{1}{p+1}|u|^{p+1}|\lambda|^{p+1}\right) d x .
$$

Se tomarmos $\lambda=-1$, temos

$$
J(-u)=\int_{\Omega}\left(\frac{1}{2}(\Delta u)^{2}-|u|^{p+1}\right) d x=J(u) .
$$

Desta forma, se $u$ é o ponto mínimo global que calculamos antes, então $u \neq 0$ e

$$
J(-u)=J(u) \leq J(v), \forall v \in H_{0}^{2}(\Omega),
$$

$\operatorname{logo}-u$ é um ponto crítico de $J$ distinto de $u$. Para o problema análogo para o operador $-\Delta$, a condição $u \not \equiv 0$ é substituída por $u \geq 0$, porém como $\Delta^{2}$ não preserva positividade sob condição de Dirichlet, não podemos garantir em geral que $u$ não troca de sinal.

\subsubsection{O caso superlinear}

Suponha agora que (3.1) é subcrítico e superlinear, isto é, $p>1$. Neste caso o funcional não é limitado inferiormente nem coercivo. De fato,

$$
J(\lambda u)=\frac{1}{2}\|u\|^{2} \lambda^{2}-\frac{1}{p+1}\|u\|_{p+1}^{p+1} \lambda^{p+1},
$$

e dada $u \in \mathcal{H} \backslash\{0\}$, se $\lambda \longrightarrow \infty$, então como $p>1, J(\lambda u) \longrightarrow-\infty$.

Por outro lado, o funcional $J$ é de classe $\mathcal{C}^{2}$, conforme o Exemplo B.21. Além disso, podemos aplicar o teorema do passo da montanha, Teorema C.6 para provar a existência de uma solução fraca não-trivial. 
Teorema 3.5. Se $\Omega \subset \mathbb{R}^{N}$ é um aberto limitado, $p>1$ e o problema (3.1) é subcrítico, então existe uma solução não-trivial de (3.1) e (3.3). Se, além disso, $\partial \Omega$ tem classe $\mathcal{C}^{2}$, então existe uma solução fraca não-trivial de (3.1) e (3.5).

Demonstração. As soluções fracas são os pontos críticos do funcional

$$
J(u)=\int_{\Omega}\left(\frac{1}{2}(\Delta u)^{2}-\frac{1}{p+1}\|u\|^{p+1}\right) d x
$$

definindo em $\mathcal{H}=H_{0}^{2}(\Omega)$ ou $\mathcal{H}=H^{2}(\Omega) \cap H_{0}^{1}(\Omega)$, conforme consideremos a condição de contorno de Dirichlet ou de Navier. Provaremos que $J$ cumpre a condição $(P S)_{c}$ e as demais condições do Teorema C.6.

Inicialmente, observamos que

$$
J(u)=\frac{1}{2}\|u\|^{2}-\frac{1}{p+1}\|u\|_{p+1}^{p+1}
$$

e pela imersão contínua de $\mathcal{H}$ em $L^{p+1}(\Omega)$, temos

$$
J(u) \geq \frac{1}{2}\|u\|^{2}-\frac{1}{p+1} c\|u\|^{p+1}
$$

Escolhendo $r>0$ suficientemente pequeno, temos

$$
J(u) \geq \frac{1}{2} r^{2}-\frac{1}{p+1} C r^{p+1}>0,
$$

para qualquer $u \in \mathcal{H}$ tal que $\|u\|=r$. Assim $b=\inf _{\|u\|=r} J(u) \geq \frac{1}{2} r^{2}-\frac{1}{p+1} C r^{p+1}>0=$ $J(0)$.

Por outro lado, observando a igualdade (3.10), vemos que tomando $u \neq 0, J(\lambda u) \longrightarrow$ $-\infty$ quando $\lambda \longrightarrow \infty$. Assim podemos tomar $\lambda \neq 0$ e $e=\lambda u$, tais que $J(e)<0$ e $\|e\|>r$.

Em resumo, $r>0, e \neq 0,\|e\|>r, \mathrm{e}$

$$
J(e)<0=J(0)<b=\inf _{\|u\|=r} J(u) .
$$

Com a mesma notação do Teorema C.6, como $J$ é $\mathcal{C}^{1}(\mathcal{H})$, o Teorema C.6 nos diz que se $J$ satisfaz a condição $(P S)_{c}$, então $c$ é um nível crítico de $J$. Vamos então verificar que $J$ satisfaz a condição $(P S)_{c}$ provando o seguinte.

Se $\sup J\left(u_{k}\right)<\infty$ e $J^{\prime}\left(u_{k}\right) \longrightarrow 0$, então existe uma subsequência convergente de $\left(u_{k}\right)$. 
Observe inicialmente que para toda $u \in \mathcal{H}$, temos

$$
\begin{aligned}
J(u)-\frac{1}{p+1} J^{\prime}(u) u= & \int_{\Omega}\left(\frac{1}{2}(\Delta u)^{2}-\frac{1}{p+1}|u|^{p+1}\right) d x \\
& -\int_{\Omega}\left(\frac{1}{p+1}(\Delta u)^{2}-\frac{1}{p+1}|u|^{p-1} u u\right) d x \\
= & \left(\frac{1}{2}-\frac{1}{p+1}\right)\|u\|^{2} .
\end{aligned}
$$

Agora como $J^{\prime}\left(u_{k}\right) \longrightarrow 0$, existe $n_{0} \in \mathbb{N}$ tal que $n>n_{0}$ implica $\left\|J^{\prime}\left(u_{k}\right)\right\|<p+1, \log 0$ por (3.13),

$$
\begin{aligned}
\left\|u_{k}\right\|^{2} & =\frac{2 p+2}{p-1}\left(J\left(u_{k}\right)-\frac{1}{p+1} J^{\prime}\left(u_{k}\right) u_{k}\right) \\
& \leq \frac{2 p+2}{p-1}\left(\sup _{n \in \mathbb{N}} J\left(u_{k}\right)+\frac{1}{p+1}\left\|J^{\prime}\left(u_{k}\right)\right\|\left\|u_{k}\right\|\right) \leq \frac{2 p+2}{p-1}\left(\sup _{n \in \mathbb{N}} J^{\prime}\left(u_{k}\right)+\left\|u_{k}\right\|\right) .
\end{aligned}
$$

Assim obtemos

$$
\left\|u_{k}\right\|^{2}-\frac{2 p+2}{p-1}\left\|u_{k}\right\| \leq \sup _{n \in \mathbb{N}} J\left(u_{k}\right), \quad \forall n>n_{0}
$$

e se $\left\|u_{k}\right\| \longrightarrow \infty$, então o lado esquerdo em (3.16) fica arbitrariamente grande, contradizendo a condição sup $J\left(u_{k}\right)<\infty$. Portanto $\left(u_{k}\right)$ é limitada em $\mathcal{H}$, e existem uma subsequência, ainda denotada $\left(u_{k}\right)$, e $u \in \mathcal{H}$, tais que

$$
u_{k} \rightarrow u \quad \text { em } \mathcal{H} \text { e } u_{k} \longrightarrow u \text { em } L^{p+1}(\Omega)
$$

Vamos provar que $u_{k} \longrightarrow u$ em $\mathcal{H}$, usando a igualdade

$$
\|u-v\|^{2}=\left(J^{\prime}(u)-J^{\prime}(v)\right)(u-v)+\int_{\Omega}\left(|u|^{p+1} u-|v|^{p+1} v\right)(u-v) d x
$$

De fato, como $u_{k} \rightarrow u$, temos $J^{\prime}(u)\left(u_{k}-u\right) \longrightarrow 0$, pois $J^{\prime}(u) \in \mathcal{H}^{\prime}$. Como $\left(u_{k}\right)$ é limitada e por hipótese $J^{\prime}\left(u_{k}\right) \longrightarrow 0$, temos $\left|J^{\prime}\left(u_{k}\right)\left(u_{k}-u\right)\right| \leq\left\|J^{\prime}\left(u_{k}\right)\right\|\left\|u_{k}-u\right\|$, e assim $J^{\prime}\left(u_{k}\right)\left(u_{k}-u\right) \longrightarrow 0$. Por fim, vamos verificar que

$$
\int_{\Omega}\left(\left(\left|u_{k}\right|^{p-1} u_{k}-|u|^{p+1} u\right)\left(u_{k}-u\right)\right) d x \leq\left\|\left|u_{k}\right|^{p-1} u_{k}-|u|^{p-1} u\right\|_{q}\left\|u_{k}-u\right\|_{p+1}
$$

onde $q=\frac{p+1}{p}$. 
Como $u, u_{k} \in L^{p+1}(\Omega)$, então $|u|^{p},\left|u_{k}\right|^{p} \in L^{q}(\Omega)$ para todo $k$ e

$$
\begin{aligned}
\int_{\Omega}\left|\left(|u|^{p-1} u-\left|u_{k}\right|^{p-1} u_{k}\right)\right|^{\frac{p+1}{p}} d x & \leq \int_{\Omega}\left|\left(|u|^{p}+\left|u_{k}\right|^{p}\right)\right|^{\frac{p+1}{p}} d x \\
& =\left\||u|^{p}+\left|u_{k}\right|^{p}\right\|_{q}^{q} .
\end{aligned}
$$

Agora $\left(u_{k}\right)$ converge em $L^{p+1}(\Omega)$, logo é limitada. Segue que $\left(|u|^{p}+\left|u_{k}\right|^{p}\right)$ é uma sequência limitada em $L^{q}(\Omega)$ e

$$
\int_{\Omega}\left(|u|^{p-1} u-\left|u_{k}\right|^{p-1} u_{k}\right) d x \longrightarrow 0
$$

Por (3.18), $\left\|u_{k}-u\right\| \longrightarrow 0$, ou seja, $u_{k} \longrightarrow u$ em $\mathcal{H}$.

Verificação de (3.18):

$$
\begin{aligned}
\left(J^{\prime}(u)-J^{\prime}(v)\right)(u-v)= & \int_{\Omega}\left((\Delta u)^{2}-|u|^{p-1} u^{2}\right) d x-\int_{\Omega}\left(\Delta u \Delta v-|u|^{p-1} u v\right) d x+ \\
& +\int_{\Omega}\left((\Delta v)^{2}-|v|^{p-1} v^{2}\right) d x-\int_{\Omega}\left(\Delta v \Delta u-|v|^{p-1} v u\right) d x \\
= & \int_{\Omega}\left((\Delta u)^{2}+(\Delta v)^{2}-2 \Delta u \Delta v\right) d x+\int_{\Omega}\left(|u|^{p-1} u(u-v)+\right. \\
& \left.-|v|^{p-1} v(u-v)\right) d x \\
= & \int_{\Omega}(\Delta(u-v))^{2} d x-\int_{\Omega}\left(|u|^{p-1} u-|v|^{p-1} v\right)(u-v) d x
\end{aligned}
$$

Outra maneira de obter uma solução para o problema (3.1) é por minimização em um conjunto limitado. Considere inicialmente $S=\left\{u \in H_{0}^{2}(\Omega) ; \int_{\Omega}|u|^{p+1} d x=1\right\}$, observe que $S=K^{-1}(1)$, onde $K$ é o funcional do exemplo B.21,

$$
K(u)=\int_{\Omega}|u|^{p+1} d x
$$

O funcional $M(u)=\frac{1}{2}\|u\|^{2}$ é limitado inferiormente por 0 e coercivo, considere $c=$ $\inf _{S} M(u) \geq 0$. Tome $\left(u_{k}\right)$ uma sequência minimizante de $M$ em $S$, isto é, $M\left(u_{k}\right) \longrightarrow$ c. Pela coercividade de $M$, a sequência $\left(u_{k}\right)$ não pode ser ilimitada. De fato, neste caso existiria uma subsequência $\left(u_{k_{j}}\right)$ tal que $\left\|u_{k_{j}}\right\| \longrightarrow \infty$ e assim $M\left(u_{k_{j}}\right) \longrightarrow \infty$, contradizendo $M\left(u_{k}\right) \longrightarrow c$. A sequência $\left(u_{k}\right)$ possui então uma subsequência convergente, ainda denotada $\left(u_{k}\right)$. Seja $u \in \mathcal{H}$ tal que $\left(u_{k}\right) \longrightarrow u$, como $M$ é semicontínuo inferiormente por sequências na topologia fraca de $\mathcal{H}, M(u) \leq \liminf M\left(u_{k}\right)=c$.

Por outro lado, como (3.1) é subcrítico, temos imersão compacta de $\mathcal{H}$ em $L^{p+1}(\Omega)$, 
assim existe uma subsequência de $\left(u_{k}\right)$ tal que $u_{k} \longrightarrow u$ em $L^{p+1}(\Omega)$. Em particular, $1=\left\|u_{k}\right\|_{p+1} \longrightarrow\|u\|_{p+1}$, assim $u \in S$ e $u$ é um ponto mínimo de $\left.M\right|_{S}$.

Para $v \in S$, temos

$$
K^{\prime}(v) v=(p+1) \int_{\Omega}|v|^{p-1} v v d x=(p+1) \int_{\Omega}|v|^{p+1} d x=p+1 \neq 0
$$

$\log K^{\prime}(v) \neq 0$ e 1 é um valor regular de $K$. Pelo Teorema C.3, existe um multiplicador de Lagrange $\lambda>0$, tal que $M^{\prime}(u)=\lambda K^{\prime}(u)$, ou seja,

$$
\int_{\Omega} \Delta u \Delta v d x=\lambda \int_{\Omega}|u|^{p-1} u v d x
$$

para qualquer $v \in \mathcal{H}$.

Tomando $w=\lambda^{\frac{1}{p-1}} u$, obtemos por (3.20)

$$
\begin{aligned}
\int_{\Omega} \Delta w \Delta v d x & =\lambda^{\frac{1}{p-1}} \int_{\Omega} \Delta u \Delta v d x=\lambda^{\frac{1}{p-1}} \lambda \int_{\Omega}|u|^{p-1} u v d x \\
& =\int_{\Omega}\left|\lambda^{\frac{1}{p-1}} u\right|^{p-1}\left(\lambda^{\frac{1}{p-1}} u\right) v d x=\int_{\Omega}|w|^{p-1} w v d x
\end{aligned}
$$

e $\operatorname{assim} w$ é uma solução fraca de (3.1). Como $u \in S$ e $\lambda \neq 0$, temos $w \neq 0$.

\subsubsection{Solução de energia mínima}

Uma solução $u \in \mathcal{H}$ do problema (3.1) é dita de energia mínima se $u \neq 0$ e

$$
J^{\prime}(v)=0 \text { implica } J(v) \geq J(u),
$$

onde $J$ é o funcional de energia associado ao problema definido em (3.7).

Nesta Seção estudamos existência e propriedades de soluções de energia mínima do problema (3.1), completado com as condições de contorno de Dirichlet e de Navier.

Note inicialmente que se uma solução $u \neq 0$ é um ponto mínimo global, então ela é uma solução de energia mínima. Desta forma as soluções obtidas na Seção 3.2.1 para (3.1) com $0<p<1$ são de energia mínima. Por esta razão, nos restringiremos a provar a existência no caso $p>1$.

Se $u \in \mathcal{H}$ é um ponto crítico de $J$, então em particular $J^{\prime}(u) u=0$, definimos

$$
\mathcal{N}=\left\{u \in \mathcal{H} \backslash\{0\} ; J^{\prime}(u) u=0\right\}
$$

chamada variedade Nehari de $J$. Vemos que todos os pontos críticos de $J$ pertencem a 
$\mathcal{N}$, de modo que

$$
c \geq \inf _{u \in \mathcal{N}} J(u)
$$

onde $c$ é o nível de energia mínima de $J$. Veremos a seguir que para o problema (3.1) o nível de energia mínima é dado pelo termo à direita de (3.24).

Teorema 3.6. Se $\Omega \subset \mathbb{R}^{N}$ é um aberto limitado, $p>1$ (3.1) e (3.3) é subcrítico, então existe uma solução de energia minima $u_{0} \in \mathcal{N} \subset \mathcal{H}$, tal que

$$
J\left(u_{0}\right)=\inf _{u \in \mathcal{N}} J(u)
$$

Se $\partial \Omega$ é de classe $\mathcal{C}^{2}$, então existe uma solução de energia mínima $u_{0} \in H^{2}(\Omega) \cap H_{0}^{1}(\Omega)$, ponto minimo de $J$ restrito a $\mathcal{N}$.

Demonstração. Considere o problema (3.1) com condição de contorno (3.3) ou (3.5) e defina $\mathcal{H}=H_{0}^{2}(\Omega)$ ou $\mathcal{H}=H^{2}(\Omega) \cap H_{0}^{1}(\Omega)$, conforme a condição de contorno em questão. Relembrando a definição de $J$,

$$
J(u)=\int_{\Omega}\left(\frac{1}{2}(\Delta u)^{2}-\frac{|u|^{p+1}}{p+1}\right) d x,
$$

se $u \in \mathcal{N}$, então $J^{\prime}(u) u=0$ e

$$
\int_{\Omega}(\Delta u)^{2} d x=\int_{\Omega}|u|^{p+1} d x
$$

$\log 0$

$$
J(u)=\left(\frac{1}{2}-\frac{1}{p+1}\right) \int_{\Omega}(\Delta u)^{2} d x \geq 0 .
$$

Definimos

$$
c=\inf _{u \in \mathcal{N}} J(u) \geq 0
$$

tome uma sequência minimizante, isto é, $\left(u_{k}\right) \in \mathcal{N}$ e $J\left(u_{k}\right) \longrightarrow c$. Suponha que $\left(u_{k}\right)$ não é limitada, então existe uma subsequência, ainda denotada $\left(u_{k}\right)$, tal que $\left\|u_{k}\right\| \longrightarrow \infty$. Assim a igualdade (3.26) nos diz que $J\left(u_{k}\right) \longrightarrow \infty$, uma contradição. Assim $\left(u_{k}\right)$ é limitada e possui uma subsequência $\left(v_{k}\right)$ convergente na topologia fraca de $\mathcal{H}$, ou seja, existe $u_{0} \in \mathcal{H}$ tal que $v_{k} \rightarrow u_{0}$.

Como o problema é subcrítico, a imersão de $\mathcal{H}$ em $L^{p+1}(\Omega)$ é compacta. Assim a sequência limitada $\left(v_{k}\right)$ possui uma subsequência $\left(w_{k}\right)$ convergente em $L^{p+1}(\Omega)$. Em particular,

$$
\int_{\Omega}\left|w_{k}\right|^{p+1} d x \longrightarrow \int_{\Omega}\left|u_{0}\right|^{p+1} d x
$$


Pela Proposição B.4, temos

$$
\liminf _{k \rightarrow \infty} \int_{\Omega}\left(\Delta w_{k}\right)^{2} d x \geq \int_{\Omega}\left(\Delta u_{0}\right)^{2} d x
$$

De (3.27) e (3.28),

$$
\begin{aligned}
\liminf _{k \rightarrow \infty} J\left(w_{k}\right) & =\liminf _{k \rightarrow \infty} \int_{\Omega}\left(\Delta w_{k}\right)^{2} d x-\lim _{k \rightarrow \infty} \int_{\Omega}\left|w_{k}\right|^{p+1} d x \\
& \geq \int_{\Omega}\left(\Delta u_{0}\right)^{2} d x-\int_{\Omega}\left|u_{0}\right|^{p+1} d x=J\left(u_{0}\right),
\end{aligned}
$$

$\operatorname{mas} \lim _{k \rightarrow \infty} J\left(w_{k}\right)=c, \operatorname{logo} J\left(u_{0}\right)=c$.

Vamos verificar que $u_{0}$ é um ponto crítico de $J$. Seja $v \in \mathcal{H} \backslash\{0\}$, suponha que $v=\lambda u_{0}$, para algum $\lambda \in \mathbb{R}$. Então

$$
J^{\prime}\left(u_{0}\right) v=J^{\prime}\left(u_{0}\right) \lambda u_{0}=\lambda J^{\prime}\left(u_{0}\right) u_{0}=0,
$$

pois $u_{0} \in \mathcal{N}$.

Se, por outro lado, não existe $\lambda \in \mathbb{R}$ tal que $v=\lambda u_{0}$, definimos para $s \in(-\varepsilon, \varepsilon)$,

$$
f_{v}(s)=J\left(t\left(u_{0}+s v\right)\left(u_{0}+s v\right)\right)
$$

onde $t(w)$ é definido pelo Lema 3.8 a seguir. Ainda pelo Lema 3.8, 0 é um mínimo local de $f_{v}$, pois $t\left(u_{0}\right)=1$, já que $u_{0} \in \mathcal{N}$.

Podemos calcular

$$
f_{v}^{\prime}(s)=J^{\prime}\left(t\left(u_{0}+s v\right)\left(u_{0}+s v\right)\right)\left(t^{\prime}\left(u_{0}+s v\right) v\left(u_{0}+s v\right)+t\left(u_{0}+s v\right) v\right),
$$

que nos dá

$$
\begin{aligned}
f_{v}^{\prime}(0) & =J^{\prime}\left(u_{0}\right)\left(t^{\prime}\left(u_{0}\right) v\left(u_{0}\right)+t\left(u_{0}\right) v\right) \\
& =\left(t^{\prime}\left(u_{0}\right) v\right) J^{\prime}\left(u_{0}\right) u_{0}+t\left(u_{0}\right) J^{\prime}\left(u_{0}\right) v=t\left(u_{0}\right) J^{\prime}\left(u_{0}\right) v=0 .
\end{aligned}
$$

Por fim $t\left(u_{0}\right)=1$ e $J^{\prime}\left(u_{0}\right) v=0$, como queríamos. Portanto $u_{0}$ é um ponto crítico de $J$.

Observação 3.7. Se $\gamma(s)=t\left(u_{0}+s v\right)\left(u_{0}+s v\right)$, então $\gamma^{\prime}(0)=t^{\prime}\left(u_{0}\right) v\left(u_{0}\right)+t\left(u_{0}\right) v$. Se $\gamma^{\prime}(0)=0$, então pondo $\lambda=t^{\prime}\left(u_{0}\right) v \in \mathbb{R}$, como $t\left(u_{0}\right)=1$, chegamos a $\lambda u_{0}+v=0$, uma contradição. Assim $\gamma^{\prime}(0) \neq 0$ e $\gamma$ é uma curva definida em um aberto em torno de $0 \in \mathbb{R}$. 
O seguinte Lema de Willem [20, Lemma 4.1] mostra que $\mathcal{N}$ é homeomorfa à esfera de $\mathcal{H}$, logo divide $\mathcal{H}$ em duas componentes.

Lema 3.8. Se $u \in \mathcal{H} \backslash\{0\}$, então existe um único $t(u) \in(0, \infty)$, tal que $t(u) \in \mathcal{N}$. $A$ função $u \longmapsto t(u)$ é contínua. Além disso, $\psi: S \longrightarrow \mathcal{N}$, definida por $\psi(u)=t(u) u$ define um homeomorfismo da esfera $S=\{u \in \mathcal{H} ;\|u\|=1\}$ em $\mathcal{N}$.

Demonstração. Tome $u \in \mathcal{H} \backslash\{0\}$, defina a função $g(t)=J(t u)$, para $t \in(0, \infty)$. Temos então

$$
g^{\prime}(t)=J^{\prime}(t u) u, \forall t \in(0, \infty),
$$

e $\operatorname{assim} g^{\prime}(t)=0$ é equivalente a $t u \in \mathcal{N}$.

Como temos $J$ dado por (3.25), podemos calcular

$$
g^{\prime}(t)=t \int_{\Omega}(\Delta u)^{2} d x-|t|^{p-1} t \int_{\Omega}|u|^{p+1} d x
$$

assim definindo

$$
t(u)=\left(\frac{\int_{\Omega}(\Delta u)^{2} d x}{\int_{\Omega}|u|^{p+1}}\right)^{\frac{1}{p-1}}
$$

temos $g^{\prime}(t)>0$ para $t<t(u)$ e $g^{\prime}(t)<0$ para $t>t(u)$. Além disso, $t(u)$ é o máximo de $g$ em $(0, \infty)$, de modo que $g^{\prime}(t(u))=0$ e $t(u) u \in \mathcal{N}$.

Para provar a continuidade de $t$, seja $\left(u_{k}\right) \in \mathcal{H} \backslash\{0\}$ tal que $u_{k} \longrightarrow u \in \mathcal{H} \backslash\{0\}$. Claramente $\left\|u_{k}\right\|^{2} \longrightarrow\|u\|^{2}$, enquanto pela imersão de $\mathcal{H}$ em $L^{p+1}(\Omega),\left\|u_{k}\right\|_{p+1} \longrightarrow$ $\|u\|_{p+1}$. Vemos então por (3.32) que, como $u \neq 0, t(u)$ é bem definido e

$$
t\left(u_{k}\right) \longrightarrow t(u)
$$

Definindo $S=\{u \in \mathcal{H} \backslash\{0\} ;\|u\|=1\}$ e $\psi: S \longrightarrow \mathcal{N}$ por $\psi(u)=t(u) u$, temos a continuidade da aplicação $\psi$ pela continuidade de $t$. A inversa de $\psi$ é

$$
u \longmapsto \frac{u}{\|u\|}
$$

que é contínua em $\mathcal{H} \backslash\{0\}$. Portanto $\psi$ é um homeomorfismo entre $S$ e $\mathcal{N}$.

Seguindo Willem [20], provamos a seguir que o nível de energia mínima para (3.1) é o nível do passo da montanha. 
Teorema 3.9. Se $\Omega \subset \mathbb{R}^{N}$ é um aberto limitado, $p>1$, (3.1) é subcrítico,

$$
\begin{gathered}
c_{1}=\inf _{u \in \mathbb{N}} J(u), \\
c_{2}=\inf _{u \in H_{0}^{2}(\Omega) \backslash\{0\}} \max _{t \geq 0} J(t u), \\
c=\inf _{\gamma \in \Gamma} \max _{t \in[0,1]} J(\gamma(t)),
\end{gathered}
$$

onde $\Gamma=\left\{\gamma \in \mathcal{C}\left([0,1], x \in H_{0}^{2}(\Omega)\right) ; \gamma(0)=0, J(\gamma(1))<0\right\}$, então

$$
c_{1}=c_{2}=c>0
$$

e c é um valor crítico de J.

Demonstração. Pelo Lema 3.12, dada $u \neq 0$, o máximo de $J(t u)$, com $t \geq 0$ é obtido em $t=t(u)$. Logo $t(u) u \in \mathcal{N}$ e $c_{1}=c_{2}$.

Como para $t \longrightarrow \infty, J(t u) \longrightarrow-\infty$, definindo $\gamma_{u}(t)=C$ tu obtemos uma curva $\gamma_{u} \in \Gamma$, onde $C>0$ é suficientemente grande para que $J(C u)<0$. Assim $c \leq c_{2}$.

Para concluir vamos mostrar que $c_{1} \leq c$, mostrando que qualquer $\gamma \in \Gamma$ passa por $\mathcal{N}$. De fato, se $u \in H_{0}^{2}(\Omega) \backslash\{0\}$ e $\|u\|<t(u)$, então $J(u)>0$, pois $J^{\prime}(t u) u=g^{\prime}(t) \geq 0$ para $t \leq t(u)$. Logo se $\gamma \in \mathcal{C}\left([0,1], H_{0}^{1}(\Omega)\right)$ e para todo $s \in[0,1], t(\gamma(s))>\|\gamma(s)\|$, então $\gamma(1)>0$, e $\gamma \notin \Gamma$. Dada $\gamma \in \Gamma$, necessariamente existe $s \in(0,1)$ tal que $\|\gamma(s)\|>t(\gamma(s))$, portanto existe $s^{\prime} \in(0, s)$ tal que $\gamma\left(s^{\prime}\right) \in \mathcal{N}$. Isto implica $c_{1} \leq c$, como queríamos..

Vamos agora aplicar o método de decomposição em cones duais para provar que a solução de energia mínima é não-negativa quando $\Delta^{2}$ sob condição de contorno $\mathcal{B}$ preserva positividade em $\Omega$. Para isto, usaremos uma pequena variação de [6, Lemma 2.3].

Lema 3.10. Seja $\Omega \subset \mathbb{R}^{N}$ um aberto limitado com fronteira de classe $\mathcal{C}^{2}$. Se $p>1$ e (3.1) é subcrítico,

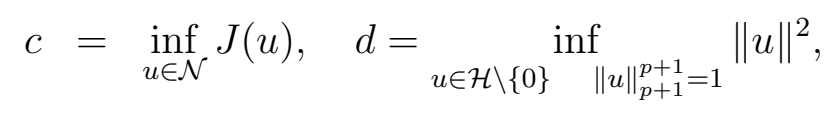

então

$$
c=\frac{p}{2(p+1)} d^{\frac{p+1}{2 p}}
$$

Além disso,

(i) $S e\left(u_{k}\right) \in \mathcal{N}$ é uma sequência minimizante de c, então $\left(u_{k} /\left\|u_{k}\right\|_{p+1}\right)$ é uma sequência minimizante para $d$, isto é, $J\left(u_{k} /\left\|u_{k}\right\|_{p+1}\right) \longrightarrow d$. 
(ii) Se $\left(u_{k}\right)$ é uma sequência minimizante de $d$, então $\left(t\left(u_{k}\right) u_{k}\right) \in \mathcal{N}$ é uma sequência minimizante para $c$.

Demonstração. Veja [6]. Observe que se $J^{\prime}(u) u=0$, então $\|u\|^{2}=\|u\|_{p+1}^{p+1}$, assim $J(u)=\left(\frac{1}{2}-\frac{1}{p+1}\right)\|u\|^{2}$. Desta forma,

$$
\frac{\int_{\Omega} \Delta^{2} u d x}{\int_{\Omega} u^{p+1} d x}=\frac{\|u\|^{2}}{\|u\|_{p+1}^{p+1}}=\frac{\|u\|^{2}}{\|u\|^{\frac{2}{p+1}}}=\|u\|^{2\left(\frac{p}{p+1}\right)}=\left(\frac{2(p+1)}{p} J(u)\right)^{\frac{2 p}{p+1}}
$$

O Lema acima permite mostrar o seguinte resultado de positividade.

Teorema 3.11. Seja $p>1$ tal que o problema (3.1) é subcrítico. Se $\mathcal{B}$ é a condição de Dirichlet (3.3) e $\Omega=B \subset \mathbb{R}^{N}$, então qualquer solução de energia minima tem sinal definido. Se $\Omega$ é um aberto limitado com fronteira de classe $\mathcal{C}^{2}$ e $\mathcal{B}$ é a condição de Navier (3.5), então qualquer solução de energia minima tem sinal definido.

Demonstração. Sejam $a \in \mathcal{H} \backslash\{0\}$ uma solução de energia mínima e $u=\frac{a}{\|a\|_{p+1}} \in \mathcal{H}$, pelo lema anterior temos

$$
\|u\|^{2}=\inf _{v \in V}\|v\|^{2}
$$

onde $V=\left\{v \in \mathcal{H} \backslash\{0\} ;\|v\|_{p+1}^{p+1}=1\right\}$. Suponha por contradição que $u$ muda de sinal. Escrevendo $u=v+w$, com $v \in K$ e $w \in K^{*}$ conforme o Corolário (2.21), temos $v \neq 0$ e $w \neq 0$. Além disso, tomando

$$
\widetilde{u}=v-w,
$$

temos $\widetilde{u} \geq u$ em $\Omega$ e $\widetilde{u}>u$ em um conjunto de medida positiva.

Agora por ortogonalidade

$$
\|u\|^{2}=(v+w, v+w)=(v, v)+(w, w)=(v-w, v-w)=\|\widetilde{u}\|^{2},
$$

enquanto por outro lado

$$
1=\int_{\Omega}|u|^{p+1} d x<\int_{\Omega}|\widetilde{u}|^{p+1} d x .
$$

Assim, $\left\|\frac{\widetilde{u}}{\|\widetilde{u}\|_{p+1}}\right\|^{2}=\frac{\|\widetilde{u}\|^{2}}{\|\widetilde{u}\|_{p+1}^{2}}=\frac{\|u\|^{2}}{\|\widetilde{u}\|_{p+1}^{2}}<\|u\|^{2}$, uma contradição com a escolha de $u$.

Portanto $u$ não muda de sinal e pelo Lema 3.10, a solução de energia mínima $a$ é um múltiplo positivo de $u, a$ é não-negativa. 


\subsection{Resultados de não-existência}

Para provar a não-existência de soluções clássicas não-triviais para 3.1 quando o domínio é estrelado e o problema é supercrítico, vamos usar o seguinte lema de [17, Corollary 2.1].

Lema 3.12. Se $u, v, w \in \mathcal{C}^{2}(\bar{\Omega})$, então

$$
\begin{aligned}
\int_{\Omega}(\Delta u(D v, D w)+\Delta v(D u, D w)) d x= & \int_{\Omega}(D u, D v) \Delta w d x+\int_{\partial \Omega}\left(\frac{\partial u}{\partial \nu}(D v, D w)+\right. \\
& \left.+\frac{\partial v}{\partial \nu}(D u, D w)-(D u, D v) \frac{\partial w}{\partial \nu}\right) d S+ \\
& +\int_{\Omega}\left(\sum_{i=1}^{N} \sum_{j=1}^{N}\left(u_{j} v_{j}+u_{j} v_{i}\right) w_{i j}\right) d x
\end{aligned}
$$

$e$

$$
\begin{aligned}
\int_{\Omega}(\Delta u(D v, x)+\Delta v(D u, x)) d x= & (N-2) \int_{\Omega}(D u, D v) d x+\int_{\partial \Omega}\left(\frac{\partial u}{\partial \nu}(x, D v)+\right. \\
& \left.+\frac{\partial v}{\partial \nu}(x, D u)-(D u, D v)(x, \nu)\right) d S
\end{aligned}
$$

Demonstração. Sejam $u, v, w \in \mathcal{C}^{2}(\bar{\Omega})$, então

$$
\int_{\Omega}(\Delta u(D v, D w)+\Delta v(D u, D w)) d x=\sum_{i=1}^{N} \sum_{j=1}^{N} \int_{\Omega}\left(u_{i i} v_{j} w_{j}+v_{i i} u_{j} w_{j}\right) d x
$$

Além disso,

$$
\begin{aligned}
\int_{\Omega}\left(u_{i i} v_{j} w_{j}+v_{i i} u_{j} w_{j}\right) d x= & \int_{\partial \Omega}\left(u_{i} \nu_{i} v_{j} w_{j}+v_{i} \nu_{i} u_{j} w_{j}\right) d S-\int_{\Omega}\left(u_{i} v_{i j} w_{j}+\right. \\
& \left.+v_{i} u_{i j} w_{j}+u_{i} v_{j} w_{i j}+v_{i} u_{j} w_{i j}\right) d x+ \\
& -\int_{\Omega}\left(u_{i} v_{i j} w_{j}+v_{i} u_{i j} w_{j}\right) d x \\
= & -\int_{\Omega}\left(u_{i} v_{i}\right)_{j} w_{j} d x=-\int_{\partial \Omega}\left(u_{i} v_{i} w_{j} \nu_{j}\right) d S+\int_{\Omega}\left(u_{i} v_{i} w_{j j}\right) d x
\end{aligned}
$$

Combinando as duas igualdades acima, obtemos (3.33). 
Quando $w=\frac{1}{2}\|x\|^{2}$, temos $w_{i}=x_{i}, w_{i j}=\delta_{i j}$ e assim

$$
\begin{aligned}
\sum_{i=1} \sum_{j=1} \int_{\Omega}\left(u_{i} v_{j} w_{i j}+u_{j} v_{i} w_{i j}\right) d x & =\sum_{i=1}^{N} \int_{\Omega}\left(u_{i} v_{i}+u_{i} v_{i}\right) d x \\
& =2 \int_{\Omega}(D u, D v) d x
\end{aligned}
$$

e

$-\sum_{i=1} \sum_{j=1} \int_{\Omega}\left(u_{i} v_{i j} w_{j}+v_{i} u_{i j} w_{j}\right) d x=-\int_{\partial \Omega}(D u, D v)(x, \nu) d S+\int_{\Omega} N(D u, D v) d x$.

Por fim, obtemos (3.34)

$$
\begin{aligned}
\int_{\Omega}(\Delta u(D v, x)+\Delta v(D u, x)) d x= & \int_{\partial \Omega}\left(\frac{\partial u}{\partial \nu}(D v, x)+\frac{\partial v}{\partial \nu}(D u, x)-(D u, D v)(x, \nu)\right) d S \\
& +(N-2) \int_{\Omega}(D u, D v) d x
\end{aligned}
$$

Vamos inicialmente aplicar (3.34) para mostrar a não-existência de solução não-trivial de (3.1) com condição de contorno de Dirichlet quando $p>\frac{N+4}{N-4}$.

Teorema 3.13. Se $N>4, p>\frac{N+4}{N-4} e \Omega \subset \mathbb{R}^{N}$ é um aberto limitado estrelado, então não existe solução clássica não-trivial de $u \in \mathcal{C}^{4}(\bar{\Omega})$ de (3.1) e (3.3).

Demonstração. Escolha $y \in \Omega$, tal que $\Omega$ é estrelado em relação a $y$, sejam $f(x)=x-y$, $\widetilde{\Omega}=f(\Omega)$, como $f$ e $f^{-1}$ são bijeções $\mathcal{C}^{\infty}$, então $u$ é solução de (3.1) se, e somente se, $\widetilde{u}=u \circ f$ é solução de

$$
\left\{\begin{aligned}
\Delta^{2} \widetilde{u} & =|\widetilde{u}|^{p-1} \widetilde{u} \text { em } \widetilde{\Omega} \\
\widetilde{u} & =\frac{\partial \widetilde{u}}{\partial \nu}=0 \quad \text { em } \partial \widetilde{\Omega}
\end{aligned}\right.
$$

Assim podemos supor que $\Omega$ é estrelado em relação a 0 .

Se $u$ cumpre a condição de Dirichlet, então o gradiente de $u$ se anula em $\partial \Omega$ e (3.34) se torna

$$
\int_{\Omega}(\Delta u(D v, x)+\Delta v(D u, x)) d x=(N-2) \int_{\Omega}(D u, D v) d x
$$

Para $v=\Delta u \in \mathcal{C}^{2}(\bar{\Omega})$, temos

$$
\int_{\Omega}\left(\Delta u(D(\Delta u), x)+\Delta^{2} u(D u, x)\right) d x=(N-2) \int_{\Omega}(D u, D(\Delta u)) d x
$$


Vamos integrar por partes os dois termos à esquerda, para o primeiro temos

$$
\int_{\Omega} \Delta u(D(\Delta u), x) d x=\int_{\partial \Omega}(\Delta u)^{2}(x, \nu) d S-N \int_{\Omega}(\Delta u)^{2} d x-\int_{\Omega} \Delta u(x, D(\Delta u)) d x
$$

$\log \mathrm{O}$

$$
\begin{aligned}
\int_{\Omega} \Delta u(D(\Delta u), x) d x & =\frac{1}{2} \int_{\partial \Omega}(\Delta u)^{2}(x, \nu) d S-\frac{N}{2} \int_{\Omega}(\Delta u)^{2} d x \\
& =\frac{1}{2} \int_{\partial \Omega}(\Delta u)^{2}(x, \nu) d S-\frac{N}{2} \int_{\Omega}|u|^{p+1} d x
\end{aligned}
$$

Usando novamente $\Delta^{2} u=|u|^{p-1} u$, o outro termo se escreve como

$$
\begin{aligned}
\int_{\Omega} \Delta^{2} u(D u, x) d x & =\int_{\Omega}|u|^{p-1} u(D u, x) d x \\
& =\frac{1}{p+1} \int_{\partial \Omega}|u|^{p+1}(x, \nu) d S-\frac{N}{p+1} \int_{\Omega}|u|^{p+1} d x
\end{aligned}
$$

Integrando por partes o último termo de (3.36), obtemos

$$
\begin{aligned}
(N-2) \int_{\Omega}(D u, D(\Delta u)) d x & =(N-2) \int_{\partial \Omega} u \frac{\partial(\Delta u)}{\partial \nu} d S-(N-2) \int_{\Omega} u \Delta^{2} u d x \\
& =-(N-2) \int_{\Omega}|u|^{p+1} d x
\end{aligned}
$$

Substituindo (3.37), (3.38) e (3.39) em (3.36), obtemos

$$
\begin{aligned}
\frac{1}{2} \int_{\partial \Omega}(\Delta u)^{2}(x, \nu) d S-\frac{N}{2} \int_{\Omega}|u|^{p+1} d x+\frac{1}{p+1} \int_{\partial \Omega}|u|^{p+1}(u, \nu) d S & -\frac{N}{p+1} \int_{\Omega}|u|^{p+1} d x \\
& =-(N-2) \int_{\Omega}|u|^{p+1} d x \\
\frac{1}{2} \int_{\partial \Omega}(\Delta u)^{2}(x, \nu) d S+\frac{1}{p+1} \int_{\partial \Omega}|u|^{p+1}(x, \nu) d S & =\left(\frac{N}{2}+\frac{N}{p+1}-(N-2)\right) \int_{\Omega}|u|^{p+1} d x \\
& =\left(\frac{N}{p+1}-\frac{N}{2}+2\right) \int_{\Omega}|u|^{p+1} d x .
\end{aligned}
$$


Observe que

$$
\begin{aligned}
\frac{N}{p+1}-\frac{N}{2}+2 & =\frac{N}{p+1}+\frac{N}{p+1}+\frac{4-N}{2}=\frac{2 N+(4-N)(p+1)}{2(p+1)} \\
& =\frac{N-N p+4 p+4}{2(p+1)}=\frac{p(4-N)+N+4}{2(p+1)} .
\end{aligned}
$$

Para $N>4, p>\frac{N+4}{N-4}$, temos $p(N-4)>N+4, \log 0>N+4+p+(N-4) \mathrm{e}$

$$
0>\frac{N+4+p(N-4)}{2 p+1}
$$

Por fim, $\frac{N}{2}+\frac{N}{p+1}-N+2<0$, portanto se $u \neq 0$, então o lado direito de (3.40) é negativo.

Como $\Omega$ é estrelado em relação à origem, $(x, \nu(x))>0$ para $x \in \partial \Omega$. Logo o lado esquerdo em (3.40) é não-negativo, o que nos leva a uma contradição.

Para o problema de Navier (3.1), podemos provar que não existe solução não-trivial não-negativa. Este resultado também é provado em [17, Theorem 3.3] e [19].

Teorema 3.14. Se $\Omega \subset \mathbb{R}^{N}$ é um aberto limitado e estrelado, $N>4, p>\frac{N+4}{N-4}$, então (3.1) não admite solução não-negativa de classe $\mathcal{C}^{4}(\bar{\Omega})$.

Demonstração. Como no Teorema 3.13 podemos supor que $\Omega$ é estrelado em relação a 0 , de modo que

$$
(x, \nu(x)) \geq 0, \quad \forall x \in \partial \Omega .
$$

Como $u=0$ na fronteira de $\Omega$, obtemos os mesmos termos nas integrais em $\Omega$ de $(3.34)$,

$$
\int_{\Omega} \Delta u(D(\Delta u), x) d x=\frac{1}{2} \int_{\partial \Omega}(\Delta u)^{2}(x, \nu) d S-\frac{N}{2} \int_{\Omega} u \Delta^{2} u d x .
$$

Como $\Delta u=0$ em $\partial \Omega$, o primeiro termo à direita de (3.42) zera. Usando $\Delta^{2} u=$ $|u|^{p-1} u$, temos

$$
\int_{\Omega} \Delta u(D(\Delta u), x) d x=-\frac{N}{2} \int_{\Omega}|u|^{p+1} d x
$$

Por outro lado

$$
\int_{\Omega} \Delta^{2} u(D u, x) d x=-\frac{1}{p+1} \int_{\partial \Omega}|u|^{p+1}(x, \nu) d S-\frac{N}{p+1} \int_{\Omega}|u|^{p+1} d x .
$$


Por fim,

$$
(N-2) \int_{\Omega}(D u, D(\Delta u)) d x=-(N-2) \int_{\Omega}|u|^{p+1} d x
$$

Como não temos $\frac{\partial u}{\partial \nu}=0$ em $\partial \Omega$, precisamos rearranjar os termos de fronteira em (3.34). Para isso observamos que como $u=\Delta u=0$ em $\partial \Omega$, temos

$$
D u=\lambda \nu, \quad D(\Delta u)=\mu \nu \text { em } \partial \Omega
$$

Como $u>0$ em $\Omega$, então

$$
D u=-\|D u\| \nu
$$

Se $u>0$, então $|u|^{p-1} u>0$ em $\Omega$, ou seja $\Delta(\Delta u)>0$ em $\Omega$. Pelo princípio do máximo fraco, Corolário A.19, $\Delta u<0$ em $\Omega$, desta forma

$$
D(\Delta u)=\|D(\Delta u)\| \nu
$$

Usando (3.46) e (3.47), o termo de fronteira se escreve

$$
\begin{aligned}
(D u, \nu)(x, D(\Delta u)) & +(D(\Delta u), \nu)(x, D u)-(D u, D(\Delta u))(x, \nu)= \\
& =(x, \nu)((D u, \nu)\|D(\Delta u)\|+(D(\Delta u), \nu)(-\|D u\|)+(-D u, D(\Delta u)) \\
& =(u, \nu)(-\|D u\|\|D(\Delta u)\|-\|D(\Delta u)\|\|D u\|+\|D u\| \| D(\Delta u \|) \\
& =-(x, \nu)(\|D u\|\|D(\Delta u)\|) .
\end{aligned}
$$

Substituindo (3.43), (3.44), (3.45), (3.48) em (3.42), obtemos

$$
\begin{aligned}
-\frac{N}{2} \int_{\Omega}|u|^{p+1} d x+ & \frac{1}{p+1} \int_{\partial \Omega}(x, \nu) d S-\frac{N}{p+1} \int_{\Omega}|u|^{p+1} d x= \\
& =-(N-2) \int_{\Omega}|u|^{p+1} d x+\int_{\partial \Omega}-\|D u\|\|D(\Delta u)\|(x, \nu) d S .
\end{aligned}
$$

Separando os termos de fronteira dos termos em $\Omega$, temos

$$
\int_{\partial \Omega}\left(\frac{1}{p+1}|u|^{p+1}+\|D u\|\|D(\Delta u)\|\right)(u, \nu) d S=\left(\frac{N}{p+1}-\frac{N+4}{2}\right) \int_{\Omega}|u|^{p+1} d x
$$

Novamente o lado esquerdo é não-negativo, e como se $N>4, p>\frac{N+4}{N-4}$, temos 
$(u<0)$, isto é, o lado direito de (3.50) é negativo para $u \neq 0$, isto é uma contradição.

Para o problema (3.1) com condição de Steklov, o Lema 3.12 permite mostrar o seguinte resultado de não-existência.

Teorema 3.15. Se $\Omega \subset \mathbb{R}^{N}$ é aberto, estrelado e limitado, a: $\partial \Omega \longrightarrow[0, \infty), N>4$ e $p>\frac{N+4}{N-4}$, então não existe solução clássica não-negativa de (3.1) sob condição de contorno de Steklov.

Demonstração. Podemos assumir que $\Omega$ é estrelado em relação a 0 . Tomando $u, v \in \mathcal{C}^{2}(\bar{\Omega})$ tais que $u=0$ na fronteira de $\Omega$, o Lema 3.12 nos dá

$$
\begin{aligned}
\int_{\Omega}(\Delta u(D v, x)+\Delta v(D u, x)) d x= & (N-2) \int_{\Omega}(D u, D v) d x+\int_{\partial \Omega}((D u, \nu)(x, D v)+ \\
& +(D v, \nu)(x, D u)-(D u, D v)(x, \nu)) d S
\end{aligned}
$$

Tome $u \in \mathcal{C}^{4}(\bar{\Omega})$, solução clássica de (3.1) com condição de contorno de Steklov e $v=-\Delta u$, obtemos $u, v \in \mathcal{C}^{2}(\bar{\Omega}) \mathrm{e}$

$$
\left\{\begin{array}{rlll}
-\Delta u & =v, \quad-\Delta v=|u|^{p-1} u & \text { em } \Omega \\
u & =0, \quad v=a \frac{\partial u}{\partial \nu} & \text { em } \partial \Omega
\end{array}\right.
$$

Integrando os termos de (3.51) usando (3.52), como feito no Teorema 3.14 obtemos as seguintes igualdades:

$$
\begin{aligned}
\int_{\Omega} \Delta u(D v, \nu) d x & =\frac{1}{2} \int_{\partial \Omega}(\Delta u)^{2}(u, \nu) d S-\frac{N}{2} \int_{\partial \Omega} \Delta u \frac{\partial u}{\partial \nu} d S-\frac{N}{2} \int_{\Omega} u \Delta^{2} u d x \\
& =\frac{1}{2} \int_{\partial \Omega}\left(N a\left(\frac{\partial u}{\partial \nu}\right)^{2}+a^{2}\left(\frac{\partial u}{\partial \nu}\right)^{2}(x, \nu)\right) d S+\frac{N}{2} \int_{\Omega}|u|^{p+1} d x \\
& (N-2) \int_{\Omega}(D u, D \Delta u) d x=-(N-2) \int_{\Omega}|u|^{p+1} d x
\end{aligned}
$$

Para agrupar os termos de fronteira em (3.51), observe que $u=0$ em $\partial \Omega$ e $u>0$ em $\Omega, \operatorname{assim} D u=-\|D u\| \nu$ em $\partial \Omega$.

$$
\begin{aligned}
(D u, \nu)(u, D v) & +(D v, \nu)(x, D u)-(D u, D v)(x, \nu)= \\
& =-\|D u\|(x, D v)+(D v, \nu)(x, \nu)(-\|D u\|)+\|D u\|(D v, \nu)(x, \nu) \\
& =-\|D u\|(x, D v)
\end{aligned}
$$


Unindo (3.52), (3.53), (3.54) e (3.55) em (3.51), obtemos

$$
\begin{array}{r}
\frac{1}{2} \int_{\partial \Omega}\left(N a\left(\frac{\partial u}{\partial \nu}\right)^{2}-a^{2}(x, \nu)\left(\frac{\partial u}{\partial \nu}\right)^{2}\right) d S+\frac{N}{2} \int_{\Omega}|u|^{p+1} d x-\frac{N}{p+1} \int_{\Omega}|u|^{p+1} d x= \\
=-(N-2) \int_{\Omega}|u|^{p+1} d x+\int_{\partial \Omega}-\|D u\|(x, D v) d S \\
\int_{\partial \Omega}\left(\frac{N}{2} a\left(\frac{\partial u}{\partial \nu}\right)^{2}+\frac{a^{2}}{2}\left(\frac{\partial u}{\partial \nu}\right)^{2}(x, \nu)\right) d S
\end{array}
$$

Como $u \neq 0$, se $p>\frac{N+4}{N-4}$ e $N \geq 5$, o lado direito de (3.56) é negativo, pois $u \neq 0$. Por outro lado, $a \geq 0$ e $\Omega$ é estrelado, $\operatorname{logo}(x, \nu) \geq 0$ em $\partial \Omega$. Assim o lado esquerdo de (3.56) é não-negativo, de modo que chegamos a uma contradição. Portanto não existe solução clássica não-negativa de (3.1) sob condição de Steklov. 


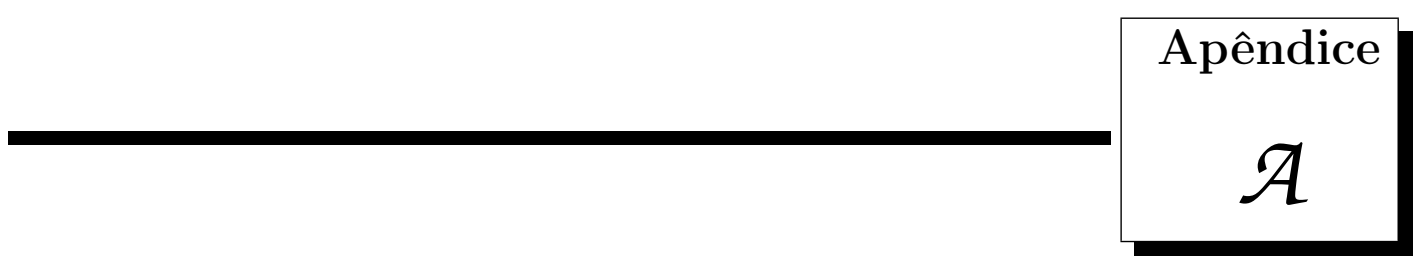

Espaços de funções

Ao longo deste capítulo $\Omega$ denota um subconjunto aberto de $\mathbb{R}^{N}$. Precisaremos dos seguintes resultados básicos.

Teorema A.1 (Teorema da convergência dominada). Se $\Omega \subset \mathbb{R}^{N}$ é um aberto, $\left(u_{k}\right)$ é uma sequência em $L^{1}(\Omega)$, tal que existe $w \in L^{1}(\Omega)$,

$$
\begin{aligned}
& u_{k}(x) \longrightarrow u(x), \text { para quase todo } x \in \Omega, \\
& \left|u_{k}(x)\right| \leq w(x), \text { para quase todo } x \in \Omega,
\end{aligned}
$$

então $u \in L^{1}(\Omega) e \int_{\Omega} u_{k} d x \longrightarrow \int_{\Omega} u d x$.

Demonstração. Veja [7].

Teorema A.2 (Desigualdade de Hölder). Se $u \in L^{p}(\Omega), v \in L^{q}(\Omega)$, e

$$
\frac{1}{p}+\frac{1}{q}=1
$$

então $u v \in L^{1}(\Omega)$, e

$$
\int_{\Omega} u v d x \leq\|u\|_{p}\|v\|_{q}
$$

Demonstração. Veja [1, Theorem 2.3].

Definição A.3. O espaço de Sobolev $H^{1}(\Omega)$ é o espaço das funções $u \in L^{2}(\Omega)$ que 
possuem derivadas fracas de ordem $1 \mathrm{em} L^{2}(\Omega)$, munido do produto interno

$$
(u, v)_{1}=\int_{\Omega}(u v+(D u, D v)) d x
$$

e da norma induzida

$$
\|u\|_{H^{1}}=\left(\int_{\Omega} u^{2}+\|D u\|^{2} d x\right)^{\frac{1}{2}} .
$$

O suporte de uma função $\varphi \in \mathcal{C}(\Omega)$ é o conjunto $\operatorname{supp} \varphi=\overline{\{x \in \Omega ; \varphi(x) \neq 0\}}$. Dizemos que $\varphi$ tem suporte compacto se $\operatorname{supp} \varphi \subset \Omega$ é um compacto.

Definição A.4. O espaço das funções de teste $\mathcal{C}_{c}^{\infty}(\Omega)$ é o conjunto das funções $\varphi: \Omega \longrightarrow$ $\mathbb{R}$ de classe $\mathcal{C}^{\infty}(\Omega)$ com suporte compacto. Uma função $\varphi \in \mathcal{C}_{c}^{\infty}(\Omega)$ é chamada função de teste.

Definição A.5. Definimos $H_{0}^{1}(\Omega)$ como o fecho de $\mathcal{C}_{c}^{\infty}(\Omega)$ na norma de $H^{1}(\Omega)$,

$$
H_{0}^{1}(\Omega)=\left\{u \in H^{1}(\Omega) ; \exists\left(u_{k}\right) \in \mathcal{C}_{c}^{\infty}(\Omega) \text {, tal que } u_{k} \longrightarrow u\right\}
$$

Teorema A.6. O produto interno

$$
(u, v)=\int_{\Omega}(D u, D v) d x
$$

induz uma norma equivalente a \|\|$_{H^{1}(\Omega)}$ em $H_{0}^{1}(\Omega)$, denotada

$$
\|u\|=\left(\int_{\Omega}\|D u\|^{2} d x\right)^{\frac{1}{2}}
$$

Demonstração. Ver [13].

Teorema A.7 (Teorema do traço). Se $\Omega$ é um aberto limitado com $\partial \Omega$ de classe $\mathcal{C}^{1}$, então existe um operador linear limitado

$$
T: W^{1, p}(\Omega) \longrightarrow L^{p}(\partial \Omega)
$$

tal que para $u \in \mathcal{C}^{0}(\bar{\Omega}), T u=\left.u\right|_{\partial \Omega} e\|T u\|_{L^{p}(\partial \Omega)} \leq C\|u\|_{W^{1, p}(\Omega)}$, para toda função $u \in$ $W^{1, p}(\Omega)$, com $C$ independente de $u$.

Demonstração. Veja [10, Theorem 1, p. 258]. 
Teorema A.8. Se $\Omega \subset \mathbb{R}^{N}$ é um aberto limitado com fronteira de classe $\mathcal{C}^{1}$ e $u \in \mathcal{H}^{1}(\Omega)$, então $u \in \mathcal{H}_{0}^{1}(\Omega)$ se, e somente se, $T u=0 \mathrm{em} L^{2}(\partial \Omega)$.

Demonstração. Veja [10, Theorem 2, p. 259].

Interpretamos uma função em $H_{0}^{1}(\Omega)$ como uma função que se anula na fronteira. Pelo teorema do traço A.8, $H_{0}^{1}(\Omega)=\operatorname{ker}(T)$, ou seja, $T(u)=0$ para toda $u \in H_{0}^{1}(\Omega)$. Pela definição do operador traço, para $u$ contínua, $T(u)=\left.u\right|_{\partial \Omega}$, logo pontualmente $u(x)=0$, para cada $x \in \partial \Omega$. Para $u \in H_{0}^{1}(\Omega)$ qualquer, dizemos que $u$ se anula na fronteira no sentido do traço.

Definição A.9. O espaço $H^{2}(\Omega)$ é o conjunto das funções $u \in H^{1}(\Omega)$ tais que para $1 \leq i \leq N$ temos $\frac{\partial u}{\partial x_{i}} \in H^{1}(\Omega)$, equipado com o produto interno ( , ) $)_{2}$ e a norma induzida, definidos por

$$
\begin{gathered}
(u, v)_{2}=\int_{\Omega}\left(u v+(D u, D v)+\sum_{i=1}^{N} \sum_{j=1}^{i} u_{i j} v_{i j}\right) d x \\
\|u\|_{H^{2}}=\left(\int_{\Omega}\left(u^{2}+\|D u\|^{2}+\sum_{i=1}^{N} \sum_{j=1}^{i}\left(u_{i j}\right)^{2}\right) d x\right)^{\frac{1}{2}} .
\end{gathered}
$$

Um espaço de Banach $X$ é dito separável se possui um subconjunto enumerável denso. Ele é dito reflexivo se $X$ existir uma bijeção linear contínua de $X$ sobre $\left(X^{\prime}\right)^{\prime}$.

Teorema A.10. Os espaços $H^{1}\left(\Omega\right.$ e $H^{2}(\Omega)$ são espaços de Hilbert reflexivos e separáveis.

Demonstração. Veja [1, Theorem 3.5]

Consideramos os seguintes subespaços de $H^{2}(\Omega)$, o espaço $H^{2}(\Omega) \cap H_{0}^{1}(\Omega)$ e seu subespaço $H_{0}^{2}(\Omega)$ definido por

$$
\left\{u \in H^{2}(\Omega) \cap H_{0}^{1}(\Omega) ; 1 \leq i \leq n \Rightarrow \frac{\partial u}{\partial x_{i}} \in H_{0}^{1}(\Omega)\right\} .
$$

Teorema A.11. Sejam $\Omega \subset \mathbb{R}^{N}$ um aberto limitado, $(u, v): H_{0}^{2}(\Omega) \times H_{0}^{2}(\Omega) \longrightarrow \mathbb{R} o$ produto interno

$$
(u, v)=\int_{\Omega} \Delta u \Delta v d x
$$

e \| $\|$ a norma induzida, denotada

$$
\|u\|=\int_{\Omega}(\Delta u)^{2} d x
$$


A norma \|\| é equivalente a \|\|$_{H^{2}}$, isto é, existem $C>c>0$ tais que para toda $u \in H_{0}^{2}(\Omega)$

$$
c\|u\|_{H^{2}} \leq\|u\| \leq C\|u\|_{H^{2}} .
$$

Demonstração. [12, Theorem 2.2]

Como as normas são equivalentes, $\left(H_{0}^{2}(\Omega),\|\|\right)$ é um espaço de Hilbert. Frequentemente consideraremos esta norma em $H_{0}^{2}(\Omega)$. Em $H^{2}(\Omega) \cap H_{0}^{1}(\Omega)$ o produto interno ( , ) é bem definido, porém para obter equivalência das normas é preciso impor uma condição sobre a regularidade da fronteira de $\Omega$, conforme a definição a seguir.

Definição A.12. Um aberto $\Omega \subset \mathbb{R}^{N}$ tem fronteira de classe $\mathcal{C}^{2}$ se para cada $x \in \partial \Omega$ existem um aberto $A \subset \mathbb{R}^{N-1}$ e uma bijeção $\Psi: A \longrightarrow \partial \Omega$, tal que $x \in \Psi(A)$ e $\Psi$ é de classe $\mathcal{C}^{2}$, com inversa também de classe $\mathcal{C}^{2}$.

Teorema A.13. Se $\Omega \subset \mathbb{R}^{N}$ é um aberto com fronteira de classe $\mathcal{C}^{2}$, então

$$
(u, v)=\int_{\Omega} \Delta u \Delta v d x
$$

é um produto interno em $H^{2}(\Omega) \cap H_{0}^{1}(\Omega)$. Além disso, a norma induzida é equivalente à norma de $H^{2}(\Omega)$, isto é, existem $C>c>0$ tais que

$$
c\|u\|_{H^{2}(\Omega)} \leq\|u\| \leq C\|u\|_{H^{2}(\Omega)},
$$

para toda $u \in H^{2}(\Omega) \cap H_{0}^{1}(\Omega)$, onde $\|u\|=(u, u)^{\frac{1}{2}}$.

Demonstração. Ver [12, Theorem 2.31].

Observamos que o teorema também é válido quando o domínio é convexo, conforme [2]. Além disso, quando a fronteira é de classe $\mathcal{C}^{2}$ ela é lipschitziana, logo vale o Teorema A.15, conforme a Observação A.16. Veja [13].

Assim como em $H_{0}^{1}(\Omega)$, uma função $u \in H^{2}(\Omega) \cap H_{0}^{1}(\Omega)$ é uma função que se anula na fronteira no sentido do traço. Se $u \in H_{0}^{2}(\Omega)$ é de classe $\mathcal{C}^{2}(\bar{\Omega})$, então

$$
u=\frac{\partial u}{\partial \nu}=0 \text { em } \partial \Omega
$$

De fato, $\left.u\right|_{\partial \Omega}=0$ e para $1 \leq i \leq N$, temos $\frac{\partial u}{\partial x_{i}} \in \mathcal{C}(\bar{\Omega})$ e $u_{i} \in H_{0}^{1}(\Omega)$, de modo 
que $\left.\frac{\partial u}{\partial x_{i}}\right|_{\partial \Omega}=0$. Assim para $x \in \partial \Omega$ temos $D u(x)=0$. Em particular, $\frac{\partial u}{\partial \nu}(x)=$ $(D u(x), \nu(x))=0$.

Teorema A.14. Seja $\Omega \subset \mathbb{R}^{N}$ um aberto limitado. Se $N \leq 2$ e $q \geq 1$, então $H_{0}^{1}(\Omega) \subset$ $L^{q}(\Omega)$ com imersão compacta. Em particular, existe $c>0$ tal que

$$
\|u\|_{q} \leq c\|u\|, \quad \forall u \in H_{0}^{1}(\Omega)
$$

Se $N>2$ e $1 \leq q \leq \frac{2 N}{N-2}$, então $H_{0}^{1}(\Omega) \subset L^{q}(\Omega)$, com imersão compacta se $q<\frac{2 N}{N-2}$. Existe $c>0$ tal que

$$
\|u\|_{q} \leq c\|u\|, \quad \forall u \in H_{0}^{1}(\Omega) .
$$

Demonstração. Veja [13, Theorem 7.10]

Teorema A.15 (Teorema de imersão). Seja $\Omega \subset \mathbb{R}^{N}$ um aberto limitado. Se $N \leq 4$ e $q \geq 1$, então $H_{0}^{2}(\Omega) \subset L^{q}(\Omega)$ com imersão compacta. Em particular, existe $c>0$ tal que $\forall u \in H_{0}^{2}(\Omega)$,

$$
\|u\|_{q} \leq c\|u\|
$$

Se $N>4$ e $1 \leq q \leq \frac{2 N}{N-4}$, então $H_{0}^{2}(\Omega) \subset L^{q}(\Omega)$, com imersão compacta se $q<\frac{2 N}{N-4}$.

Existe $c>0$ tal que

$$
\|u\|_{q} \leq c\|u\|, \quad \forall u \in H_{0}^{2}(\Omega) .
$$

Demonstração. Veja [13, Corollary 7.11].

Observação A.16. Se adicionarmos a hipótese $\partial \Omega$ é Lipschitz, então as imersões acima valem para o espaço $H^{2}(\Omega)$ e em particular para $H^{2}(\Omega) \cap H_{0}^{1}(\Omega)$.

Observação A.17. Ainda quando a fronteira de $\Omega$ é de classe $\mathcal{C}^{2}$ o espaço $\mathcal{C}^{2}(\bar{\Omega}) \cap \mathcal{C}_{0}^{0}(\bar{\Omega})$ é denso em $H^{2}(\Omega) \cap H_{0}^{1}(\Omega)$, veja [13, p. 174].

Teorema A.18. Seja $u \in H^{1}(\Omega)$, tal que $-\Delta u \geq 0$ em $\Omega$, então

$$
\inf _{\Omega} u \geq \inf _{\partial \Omega} u^{-}
$$


Demonstração. Ver [13, Theorem 8.1].

Corolário A.19. Se $u \in H_{0}^{1}(\Omega),-\Delta u \leq 0$ em $\Omega$, então $u \geq 0$ em $\Omega$.

Demonstração. Se $u \in H^{1}(\Omega)$, então $u^{-} \in H^{1}(\Omega)$, em particular, como $u \in H_{0}^{1}(\Omega)$, também $u^{-} \in H_{0}^{1}(\Omega)$, logo $\inf _{\partial \Omega} u^{-}=0$. No Teorema A.18, $\inf _{\Omega} u \geq 0, \operatorname{assim} u \geq 0$ em $\Omega$. 


\section{Apêndice}

\section{$\mathcal{B}$}

\section{Cálculo em espaços de Banach}

\section{B.1 Funcionais diferenciáveis}

Seja $X$ um espaço de Banach, denotamos $X^{\prime}$ ao espaço dual de $X$.

Definição B.1. Um funcional é uma aplicação $J: A \longrightarrow \mathbb{R}$, onde $A$ é um subconjunto não-vazio de $X$. Dizemos que $J$ é contínuo se ele é contínuo na topologia forte de $X$, isto é, se $\left(u_{k}\right) \in A, u_{k} \longrightarrow u \in A$, então

$$
J\left(u_{k}\right) \longrightarrow J(u)
$$

Outra noção de continuidade útil é a de semicontinuidade.

Definição B.2. Um funcional J é semicontínuo inferiormente por sequências na topologia fraca de $X$ se para toda sequência $\left(u_{k}\right) \in A$, tal que $u_{k} \rightarrow u \in A$,

$$
\liminf J\left(u_{k}\right) \geq J(u)
$$

Definição B.3. Dizemos que $J$ é coercivo se para todo $M \in \mathbb{N}$, existir $R>0$ tal que $\|u\|>R$ implica $J(u)>M$.

Proposição B.4. Se $\mathcal{H}$ é um espaço de Hilbert, então o funcional J definido por $J(u)=$ $\|u\|$ é semicontínuo inferiormente por sequências na topologia fraca de $\mathcal{H}$.

Demonstração. Sejam $u \in \mathcal{H}$, e $u_{k} \rightarrow u$, considere o funcional linear

$$
\varphi v=(u, v)
$$


$\varphi$ é limitado e $\|\varphi\|=\|u\|$.

Como $u_{k} \rightarrow u, \varphi u_{k} \longrightarrow \varphi u, \log \mathrm{o}$

$$
\varphi u=\liminf _{k \rightarrow \infty} \varphi u_{k} \leq \liminf _{k \rightarrow \infty}\|\varphi\|\left\|u_{k}\right\|=\|u\| \liminf _{k \rightarrow \infty}\left\|u_{k}\right\|
$$

onde usamos $(u, w) \leq\|v\|\|w\|$.

Temos então

$$
\|u\|^{2}=\varphi u \leq \liminf _{k \rightarrow \infty}\left\|u_{k}^{\prime}\right\|
$$

e se $u \neq 0$, temos

$$
\|u\| \leq \liminf _{k \rightarrow \infty}\left\|u_{k}\right\|
$$

Por outro lado, $\left\|u_{k}\right\| \geq 0$, assim se $u=0$, temos também $\|u\|=0 \leq \liminf _{k \rightarrow \infty}\left\|u_{k}\right\|$.

Vamos definir duas noções de diferenciabilidade de funcionais. Sejam $A \subset X e u \in A$, suponha que existe $U \subset A$ aberto tal que $u \in U$.

Definição B.5. Dizemos que $J$ tem derivada de Gâteaux em u se existe $\varphi \in X^{\prime}$, tal que

$$
h \in X \Longrightarrow \lim _{t \rightarrow 0} \frac{1}{t}[J(u+t h)-J(u)-\varphi(t h)]=0 .
$$

Definição B.6. Dizemos que $J$ tem derivada de Fréchet em $x \in U$ se existir um elemento $\varphi \in X^{\prime}$ tal que

$$
\lim _{h \rightarrow 0} \frac{1}{\|h\|}(J(u+h)-J(u)-\langle\varphi, h\rangle)=0 .
$$

Um funcional $J: A \longrightarrow \mathbb{R}$ é dito de classe $\mathcal{C}^{1}$ se sua derivada de Fréchet existe em todo ponto de $A$ e $J^{\prime}: X^{\prime} \longrightarrow \mathbb{R}$ é contínua.

Denotamos $J^{\prime}(u)$ à derivada de Gâteaux de $J$ em $u$ e $D J(u)$ à derivada de Fréchet no mesmo ponto. Se $X$ é um espaço de Hilbert e $J: A \longrightarrow \mathbb{R}$ tem derivada de Gâteaux em $u \in A$, o gradiente de $J$ em $u$ é o único elemento de $X$, denotado $\nabla J(u)$, tal que

$$
(\nabla J(u), h)=\left\langle J^{\prime}(u), h\right\rangle, \forall h \in H \text {. }
$$

Teorema B.7 (Teorema do valor médio). Sejam $X$ um espaço de Banach, $A \subset X$ um aberto, $J: A \longrightarrow \mathbb{R}$ um funcional e $u, v \in X$, tais que $[u, u+v]=\{x \in X ; x=u+t v, 0 \leq$ $t \leq 1\} \subset A$. Se J é contínuo em $[u, u+v]$ e para todo $t \in(0,1)$ existe a derivada de Gâteaux de $J$ em $u+t v$, então existe $s \in(0,1)$ tal que

$$
J^{\prime}(u+s v) v=J(u+v)-J(u) .
$$


Demonstração. Considere $f:[0,1] \longrightarrow \mathbb{R}, f(t)=J(u+t v)$. Pelas hipóteses em $J, f$ é contínua em $[0,1]$ e para cada $t \in(0,1)$, existe

$$
\begin{aligned}
J^{\prime}(u+t v) v & =\lim _{h \rightarrow 0} \frac{J(u+t v+h v)-J(u+t v)}{h} \\
& =\lim _{h \rightarrow 0} \frac{f(t+h)-f(t)}{h} \\
& =f^{\prime}(t) .
\end{aligned}
$$

Pelo teorema do valor médio para funções reais, existe $s \in(0,1)$, com

$$
\begin{gathered}
f(1)-f(0)=f^{\prime}(s)(1-0), \text { ou } \\
J(u+v)-J(u)=J^{\prime}(u+s v) v .
\end{gathered}
$$

Observação B.8. A derivada de Gâteaux é dada por

$$
J^{\prime}(u) h=\lim _{t \rightarrow 0} \frac{1}{t}(J(u+t h)-J(u)) .
$$

De fato, $\lim _{t \rightarrow 0} \frac{1}{t} J^{\prime}(u)(t h)=\lim _{t \rightarrow 0} \frac{1}{t} J^{\prime}(u) h=J^{\prime}(u) h$. Além disso, se a derivada de Gâteaux de $J$ em $u$ existe, temos

$$
\begin{aligned}
\lim _{t \rightarrow 0} \frac{1}{t}(J(u+t h)-J(u)) & =\lim _{t \rightarrow 0} \frac{1}{t}\left(J(u+t h)-J(u)-J^{\prime}(u) t h+J^{\prime}(u) t h\right) \\
& =\lim _{t \rightarrow 0} \frac{1}{t}\left(J(u+t h)-J(u)-J^{\prime}(u) t h\right)+\lim _{t \rightarrow 0} \frac{1}{t} J^{\prime}(u) t h \\
& =0+J^{\prime}(u) h .
\end{aligned}
$$

Observação B.9. Se J possui derivada de Fréchet em $u \in A$, então J possui derivada de Gâteaux e DJ $(u)=J^{\prime}(u)$.

De fato, se $u_{k} \longrightarrow 0$ em $X$, então

$$
\left.\frac{1}{t}\left(J\left(u+y_{n}\right)-J(u)-\varphi\left(y_{n}\right)\right)\right) \longrightarrow 0
$$

Tomando $h \in X, h \neq 0, t_{n} \longrightarrow 0$ em $\mathbb{R}$, temos $y_{n}=t_{n} h \longrightarrow 0$ em $X, \operatorname{logo}$

$$
\left.\frac{1}{t}\left(J\left(u+y_{n}\right)-J(u)-\varphi\left(y_{n}\right)\right)\right) \longrightarrow 0
$$


e assim

$$
\left.\lim _{t \rightarrow 0} \frac{1}{t}(J(u+t h)-J(u)-\varphi(t h))\right)=0,
$$

e $\varphi$ é a derivada de Gâteaux de $J$ em $u$.

Proposição B.10. Se $J: U \longrightarrow \mathbb{R}$ é um funcional, $U$ é um subconjunto aberto de $X$ e a derivada de Gâteaux de $J$ existe e é continua em $U$, então $J \in \mathcal{C}^{1}(U)$.

Demonstração. Tome $u_{0} \in U$. Como $J^{1}: U \longrightarrow X^{1}$ é contínuo, então o funcional $K: U \longrightarrow \mathbb{R}$ é contínuo, onde $K(u)=J(u)-J^{\prime}\left(u_{0}\right) u$. Além disso, a derivada de Gâteaux de $K$ é

$$
K(u)=J^{\prime}(u) h-J^{\prime}\left(u_{0}\right) h
$$

existe e é contínua em $U$.

Tome $h \in X$, com $0<|h|<\delta$, e $B\left(u_{0}, \delta\right) \subset U$, temos então pelo teorema do valor médio a existência de $v \in\left(u_{0}, u_{0}+h\right)$ tal que

$$
\begin{gathered}
K\left(u_{0}+h\right)-K\left(u_{0}\right)=K^{\prime}(r) h, e \\
\left|K\left(u_{0}+h\right)-K\left(u_{0}\right)\right| \leq\left|J^{\prime}(r)-J^{\prime}\left(u_{0}\right)\right||h| .
\end{gathered}
$$

- Se $H_{n} \longrightarrow 0$, a sequência $\left(v_{n}\right)$ converge para $u_{0}$, logo $\left|J^{\prime}\left(v_{n}\right)-J^{\prime}\left(u_{0}\right)\right| \longrightarrow 0$, e

$$
\frac{1}{\|h\|}\left(K\left(u_{0}+h_{n}\right)-K\left(n_{0}\right)\right) \longrightarrow 0
$$

- Dado $\varepsilon>0$, existe $\delta^{\prime}>0, \delta>\delta^{\prime}$, tal que $\left|v-u_{0}\right|<\delta^{\prime}$ implica $\left|J^{\prime}(v)-J^{\prime}\left(u_{0}\right)\right|<\varepsilon$, $\operatorname{assim}\left|K\left(u_{0}+h\right)-K\left(u_{0}\right)\right| \leq \varepsilon|h|, \mathrm{e}$

$$
\lim _{t \rightarrow 0} \frac{1}{|h|}\left(K\left(u_{0}+h\right)-K\left(u_{0}\right)\right)=0 .
$$

$\operatorname{Mas} K\left(u_{0}+h\right)-K\left(u_{0}\right)=J\left(u_{0}+h\right)-J^{\prime}\left(u_{0}\right)\left(u_{0}+h\right)-J\left(u_{0}\right)-J^{\prime}\left(u_{0}\right) u_{0}=J\left(u_{0}+\right.$ $h)-J\left(u_{0}\right)-J^{\prime}\left(u_{0}\right) h$, e assim

$$
\lim _{t \rightarrow 0} \frac{1}{|h|}\left(J\left(u_{0}+h\right)-J\left(u_{0}\right)-J^{\prime}\left(u_{0}\right) h\right)=0,
$$

e existe a derivada de Fréchet de $J$ em $u_{0}$. Assim $J$ tem derivada de Fréchet em $U$, e como ela é contínua, $J \in \mathcal{C}^{1}(U)$. 
Definição B.11. Dizemos que um funcional $J \in \mathcal{C}^{1}(U), U \subset X$ tem segunda derivada de Gâteaux em $u \in U$, se existe $L \in \mathcal{L}\left(X, X^{\prime}\right)$, tal que $\forall v, h \in X$

$$
\lim _{t \rightarrow 0} \frac{1}{t}\left(J^{\prime}\left(u_{0}+t h\right)-J^{\prime}(u) v-L(t h) v\right)=0 .
$$

Denotamos a $L=J^{\prime \prime}(u)$.

Definição B.12. Dizemos que $J$ possui segunda derivada de Fréchet se existe $L \in$ $\mathcal{L}\left(X, X^{\prime}\right)$, tal que $\forall v \in X$,

$$
\lim _{t \rightarrow 0} \frac{1}{\|h\|}\left(J^{\prime}(u+h) v-J^{\prime}(u) v-L(h) v\right)=0 .
$$

Um funcional $J$ que tem segunda derivada de Fréchet continua em U é dito de classe $\mathcal{C}^{2}(U)$

Observação B.13. Se J tem segunda derivada de Gâteaux em $u \in X$, então

$$
J^{\prime \prime}(u) v h=\lim _{t \rightarrow 0} \frac{1}{t}\left(J^{\prime}(u+h) v-J^{\prime}(u) v\right)
$$

Proposição B.14. Se $J \in \mathcal{C}^{1}(U), U \subset X$ aberto, e $J$ possui derivada de Gâteaux contínua em $U$, então $J \in \mathcal{C}^{2}(U)$.

Demonstração. Veja [20].

Proposição B.15. Se $X$ é um espaço de Banach, $J, K \in \mathcal{C}^{i}(X), i \in\{1,2\}$, então

$$
J+K \in \mathcal{C}^{i}(X)
$$

Demonstração. Veja [4].

\section{B.2 Exemplos}

Os funcionais a seguir são exemplos de funcionais de classe $\mathcal{C}^{2}$ que aparecem ao longo do texto. Seja $X$ um espaço de Banach.

Exemplo B.16. Os funcionais lineares contínuos são o primeiro exemplo de funcional. Seja $\varphi \in X^{\prime}, \varphi$ é de classe $\mathcal{C}^{2}(X)$. 
De fato, tome $\varphi \in X^{\prime}$ um funcional linear e observe que para toda $h \in X$, temos

$$
\frac{\varphi(u+t h)-\varphi(u)}{t}=\frac{\varphi(u)+t \varphi(h)-\varphi(u)}{t}=\varphi(h),
$$

logo a derivada de $\varphi$ em $u$ é $\varphi^{\prime}(u) h=\lim _{t \rightarrow 0} \varphi(h)=\varphi(h)$.

Assim $\varphi^{\prime}(u)=\varphi$, para toda $u \in X$.

Claramente $\varphi^{\prime}$ é contínuo em $X$, e portanto $\varphi \in \mathcal{C}^{1}(X)$, pela Proposição B.10. Calculando $\varphi^{\prime \prime}(u)$, para $h, k \in X$, obtemos:

$$
\lim _{t \rightarrow 0} \frac{1}{t}\left(\varphi^{\prime}(u+t h) k-\varphi^{\prime}(u) k\right)=\lim _{t \rightarrow 0} \frac{1}{t}(\varphi(k)-\varphi(k))=0 .
$$

Assim, dado $u \in X, \varphi^{\prime \prime}(u) h k=0$, para quaisquer $h, k \in X$, ou seja, $\varphi^{\prime \prime}(u)=0$. Novamente $\varphi^{\prime \prime}$ é contínuo, porque é constante.

Pela Proposição B.14, $\varphi \in \mathcal{C}^{2}(X)$.

Exemplo B.17. Seja $A: X \times X \longrightarrow \mathbb{R}$ uma forma bilinear continua, isto é,

$$
\begin{aligned}
& A(u+t v, w)=A(u, w)+t A(v, w) \\
& A(u, v+t w)=A(u, v)+t A(u, w),
\end{aligned}
$$

e além disso existe $C>0$, tal que para quaisquer $u, v \in X,|A(u, v)| \leq C\|u\|\|v\|$. O funcional $J(u)=A(u, u)$ é de classe $\mathcal{C}^{2}(X)$.

De fato, $J$ é contínuo em $X$, pois

$$
\begin{aligned}
|J(u)-J(v)| & =|A(u, u)-A(v, v)| \\
& =\frac{1}{2}|A(u+v, u-v)+A(u-v, u+v)| \\
& \leq \frac{1}{2}|A(u+v, u-v)|+\frac{1}{2}|A(u-v, u+v)| \\
& \leq \frac{1}{2} C\|u+v\|\|u-v\|+\frac{1}{2} C\|u-v\|\|u+v\| \\
& =C\|u+v\|\|u-v\|,
\end{aligned}
$$

onde usamos

$$
\begin{aligned}
& A(u+v, u-v)=A(u, u)-A(u, v)+A(v, u)-A(v, v) \\
& A(u-v, u+v)=A(u, u)+A(u, v)-A(v, u)-A(v, v) .
\end{aligned}
$$


Tomando $\left(u_{k}\right) \in X, u_{k} \longrightarrow u \in X$, então $\left\|u_{k}-u\right\| \longrightarrow 0$, logo

$$
\left|J\left(u_{k}\right)-J(u)\right| \leq C\left\|u_{k}+u\right\|\left\|u_{k}-u\right\| \longrightarrow 0,
$$

e $\operatorname{assim} J\left(u_{k}\right) \longrightarrow J(u)$

Sejam $u, h \in X$, temos

$$
\begin{aligned}
J^{\prime}(u) h & =\lim _{t \rightarrow 0} \frac{1}{t}(J(u+h)-J(u)) \\
& =\lim _{t \rightarrow 0} \frac{1}{t}(A(u+h, u+h)-A(u, u)) \\
& =A(u, h)+A(h, u) .
\end{aligned}
$$

Vamos verificar que $J^{\prime}$ é contínuo. De fato, se $u, v \in X$, então

$$
\begin{aligned}
\left\|J^{\prime}(u)-J^{\prime}(v)\right\| & =\sup _{\|h\|=1}\left|J^{\prime}(u) h-J^{\prime}(v) h\right| \\
& =\sup _{\|h\|=1}|A(u-v, h)+A(h, u-v)| \\
& \leq 2 C\|u-v\| .
\end{aligned}
$$

Isso mostra que $J$ é $\mathcal{C}^{1}(X)$, pela proposição (B.10).

Vamos calcular a segunda derivada de Gâteaux de $J$ em $u \in X$.

$$
\begin{aligned}
J^{\prime}(u+t h) k-J^{\prime}(u) k & =A(u+t h, k)+A(k, u+t h)-A(u, k)-A(k, u) \\
& =t A(h, k)+t A(k, h),
\end{aligned}
$$

$\log \mathrm{O}$

$$
\begin{aligned}
J^{\prime \prime}(u)(h, k) & =\lim _{t \rightarrow 0} \frac{1}{t}(t A(h, k)+t A(k, h)) \\
& =A(h, k)+A(k, h) .
\end{aligned}
$$

Note que $J^{\prime \prime}$ é contínuo, uma vez que é constante, e assim $J \in \mathcal{C}^{2}(X)$.

Exemplo B.18. Seja $\mathcal{H}$ um espaço de Hilbert, tomando no exemplo anterior $A(u, v)=$ $(u, v)$, temos

$$
J(u)=(u, u)=\|u\|^{2} .
$$

Pela simetria do produto interno,

$$
J^{\prime}(u) h=2(u, h), \quad J^{\prime \prime}(u)(h, k)=2(h, k) .
$$


Exemplo B.19. Se $\Omega \subset \mathbb{R}^{N}$ é aberto, então o funcional $J(u)=\int_{\Omega}\left(u_{i j} u_{k l}\right) d x$ definido em $H^{2}(\Omega)$ é de classe $\mathcal{C}^{2}$.

De fato, $A(u, v)=\int_{\Omega}\left(u_{i j} u_{k l}\right) d x$ é uma claramente uma forma bilinear. Além disso, $A$ é contínua, pois

$$
\begin{aligned}
|A(u, v)| & \leq \int_{\Omega}\left(u_{i j} u_{k l}\right) d x \\
& \leq\left\|u_{i j}\right\|_{2}\left\|u_{k l}\right\|_{2} \\
& \leq\|u\|\|v\| .
\end{aligned}
$$

O funcional $J$ é definido por $J(u)=A(u, u)$, e assim pelo Exemplo B.17, segue que $J \in \mathcal{C}^{2}\left(H^{2}(\Omega)\right)$.

Exemplo B.20. Seja $\Omega \subset \mathbb{R}^{N}$ um aberto, o funcional

$$
J(u)=\int_{\Omega}|u|^{p} d x \text { é de classe } \mathcal{C}^{1}\left(L^{p}(\Omega)\right)
$$

Observe inicialmente que, para $p>1, f_{p}(t)=|t|^{p}$ é diferenciável em $\mathbb{R} \backslash\{0\}$, e $f_{p}^{\prime}(t)=p|t|^{p-2} t$. Sejam $u, v \in L^{p}(\Omega), x \in \Omega, t$ um número real tal que $0<|t|<1$, suponha $u \neq 0, v \neq 0$. Definindo a função $f_{x}(\lambda)=|u(x)+\lambda v(x)|^{p}$, vemos que $f_{x}$ é diferenciável em $(0,|t|)$, e contínua em $[0,|t|]$. Além disso, $f_{x}^{\prime}(\lambda)=p|u(x)+\lambda v(x)|^{p-1} v(x)$. Pelo teorema do valor médio para funções reais, existe $\lambda \in(0,|t|)$ tal que

$$
t f_{x}^{\prime}(\lambda)=f_{x}(t)-f_{x}(0)
$$

ou seja,

$$
p|u(x)+\lambda v(x)|^{p-2}(u(x)+\lambda v(x)) v(x) t=|u(x)+t v(x)|^{p}-|u(x)|^{p} .
$$

Temos então uma função $\lambda: \Omega \longrightarrow(0,1)$ tal que

$$
|u(x)+t v(x)|^{p}-|u(x)|^{p}=p\left|u(x)+\lambda_{t}(x) v(x)\right|^{p-2}\left(u(x)+\lambda_{t}(x) v(x)\right) v(x) t,
$$

$\log 0$

$$
\begin{aligned}
J(u+t v)-J(u) & =\int_{\Omega}\left(|u+t v|^{p}-|u|^{p}\right) d x \\
& =\int_{\Omega}\left(p\left|u(x)+\lambda_{t}(x) v(x)\right|^{p-2}\left(u(x)+\lambda_{t}(x) v(x)\right) v(x) t\right) d x .
\end{aligned}
$$


Agora $0<\lambda_{t}(x)<|t|$ para todo $x \in \Omega$, logo

$$
\begin{aligned}
|p| u(x)+\left.\lambda_{t}(x) v(x)\right|^{p-2}(u(x)+ & \left.\lambda_{t}(x) v(x)\right) v(x) \mid \leq \\
& \leq|p| u(x)+\left.\lambda_{t}(x) v(x)\right|^{p-2}\left(u(x)+\lambda_{t}(x) v(x)\right)|| v(x) \mid .
\end{aligned}
$$

Como $v \in L^{p}(\Omega)$, basta verificar que $w(x)=|u(x)+t v(x)|^{p-2}(u(x)+t v(x)) \in L^{q}(\Omega)$, onde $\frac{1}{p}+\frac{1}{q}=1$. De fato $|w(x)|=|u(x)+t v(x)|^{p-1}$, logo

$$
\int_{\Omega}|w(x)|^{\frac{p}{p-1}} d x=\int_{\Omega}|u(x)+t v(x)|^{p} d x<\infty .
$$

Pela Desigualdade de Hölder A.2,

$$
\left|\int_{\Omega} p w(x) v(x) d x\right| \leq p\|w\|_{q}\|v\|_{p}
$$

de modo que $p w(x) v(x) \in L^{1}(\Omega)$.

Para todo $x \in \Omega$, se $t \longrightarrow 0$ então $p\left|u(x)+\lambda_{t}(x) v(x)\right|^{p-2}(u(x) v(x))$ converge para $p|u(x)|^{p-2}(u(x) v(x))$. Pelo teorema da convergência dominada A.1 segue que $t_{n} \longrightarrow 0 \mathrm{e}$ $\operatorname{assim} \frac{1}{t_{n}}\left(J\left(u+t_{n} v\right)-J(u)\right) \longrightarrow \int_{\Omega}|u|^{p-2} u v d x$. Assim $J^{\prime}(u) v=\int_{\Omega}|u|^{p-2} u v d x$, para quaisquer $u, v \in L^{p}(\Omega)$.

Por fim, $J^{\prime}(0)=0$. Sejam $v \neq 0, t \in \mathbb{R}$,

$$
\begin{aligned}
J(0+t v)-J(0) & =\int_{\Omega}\left(\frac{1}{2}(\Delta t v)^{2}-\frac{1}{p+1}|t v|^{p+1}\right) d x \\
& =t^{2} \int_{\Omega}(\Delta v)^{2} d x-|t|^{p-1} t^{2} \int_{\Omega}|v|^{p+1} d x \\
& =t\left(t\|v\|^{2}-|t|^{p-1} t\|v\|_{p+1}^{p+1}\right) .
\end{aligned}
$$

Como $p>0$, então $\frac{1}{t}(J(0+t v)-J(0)) \longrightarrow 0$ quando $t \longrightarrow 0$.

Vamos verificar a continuidade de $J^{\prime}$. Sejam $u, v, w \in L^{p}(\Omega)$,

$$
\begin{aligned}
\mid J^{\prime}(u) v-J^{\prime}(w) v & \leq \int_{\Omega}\left|\left(|u|^{p-2} u-|w|^{p-2} w\right) v\right| d x \\
& \leq\left(\left.\int_{\Omega}|| u\right|^{p-2} u-\left.|w|^{p-2} w\right|^{p^{\prime}} d x\right)^{\frac{1}{p^{\prime}}}\|v\|^{p}
\end{aligned}
$$

Suponha que $u_{k} \longrightarrow u$ em $L^{p}(\Omega)$, então existem $w \in L^{1}(\Omega)$ e uma subsequência de $\left(u_{k}\right)$ tais que $\left|u_{k}(x)\right|^{p} \longrightarrow|u(x)|^{p}$, em quase todo ponto $x \in \Omega$, e $\left|u_{k}\right|^{p} \leq w$, para todo $k$. 
Mas $\left|u_{k}\right|^{p}=\left(\left|u_{k}\right|^{p-1}\right)^{\frac{p}{p-1}}=\left(\left|u_{k}\right|^{p-2} u_{k}\right)^{\frac{p}{p-1}}, \operatorname{logo}$ como $w \geq 0$,

$$
\begin{aligned}
\left|u_{k}\right|^{p-2} u_{k} & \leq w^{\frac{p-1}{p}} \\
\left|u_{k}\right|^{p-2} u_{k}-|u|^{p-2} u & \leq w^{\frac{p-1}{p}}-|u|^{p-2} u \\
\left(\left|u_{k}\right|^{p-2} u_{k}-|u|^{p-2} u\right)^{\frac{p}{p-1}} & \leq\left(w^{\frac{p-1}{p}}-|u|^{p-2} u\right)^{\frac{p}{p-1}} .
\end{aligned}
$$

Como $w \in L^{1}(\Omega), w^{\frac{p-1}{p}} \in L^{\frac{p}{p-1}}(\Omega)$, e como $u \in L^{p}(\Omega),|u|^{p-2} u \in L^{\frac{p}{p-1}}(\Omega)$, desta forma

$$
w^{\frac{p-1}{p}}-|u|^{p-2} u \in L^{\frac{p}{p-1}}(\Omega), \quad \text { e }\left.\left.\left|w^{\frac{p-1}{p}}-\right| u\right|^{p-2} u\right|^{\frac{p}{p-1}} \in L^{1}(\Omega) .
$$

Por outro lado, $\left|u_{k}(x)\right|^{p} \longrightarrow|u(x)|^{p}$ implica $\left|u_{k}(x)\right|^{p}-|u(x)|^{p} \longrightarrow 0$, e também

$$
\left.|| u_{k}(x)\right|^{p-2} u_{k}(x)-\left.|u(x)|^{p-2} u(x)\right|^{\frac{p}{p-1}} \longrightarrow 0 .
$$

Pelo teorema da convergência dominada A.1, temos

$$
\left.\int_{\Omega}|| u_{k}(x)\right|^{p-2} u_{k}(x)-\left.|u(x)|^{p-2} u(x)\right|^{\frac{p}{p-1}} d x \longrightarrow 0
$$

e $\operatorname{assim} J^{\prime}\left(u_{k}\right) \longrightarrow J^{\prime}(u), J^{\prime}$ é contínuo.

Pela Proposição B.10, $J \in \mathcal{C}^{1}\left(L^{p}(\Omega)\right)$.

Exemplo B.21. Se $p \geq 2$, o funcional do exemplo anterior é de classe $\mathcal{C}^{2}\left(L^{p}(\Omega)\right)$.

Se $p=2$, temos o caso do exemplo (B.17),

$$
J(u)=\int_{\Omega}|u|^{2} d x=\|u\|_{2}^{2}, \quad \text { e } J \in \mathcal{C}^{2}\left(L^{2}(\Omega)\right) .
$$

Se $p>2$, então $p-2>0$, e $f_{p}$ é diferenciável também em 0 . Além disso, temos

$$
f_{p}^{\prime}(t)=p|t|^{p-2} t, \quad \forall t \in \mathbb{R}, \quad f_{p}^{\prime \prime}(t)=p(p-1)|t|^{p-2}, \quad \forall t \in \mathbb{R} \backslash\{0\}
$$

Vamos calcular a segunda derivada de Gâteaux de $J$, em $u, v, w \in L^{p}(\Omega)$.

$$
\begin{aligned}
J^{\prime}(u+t v) w-J^{\prime}(u) w & =p \int_{\Omega}\left(|u+t v|^{p-2}(u+t v) w-|u|^{p-2} u w\right) d x \\
& =p \int_{\Omega}\left(|u+t v|^{p-2}-|u|^{p-2}\right) u w d x+p t \int_{\Omega}\left(|u+t v|^{p-2} v w\right) d x .
\end{aligned}
$$


Basta verificar que

$$
\lim _{t \rightarrow 0} \frac{1}{t} p \int_{\Omega}\left(|u+t v|^{p-2}-|u|^{p-2}\right) u w d x=p(p-2) \int_{\Omega}|u|^{p-2} v w d x
$$

e

$$
\lim _{t \rightarrow 0} \frac{1}{t} p t \int_{\Omega}|u+t v|^{p-2} v w d x=p \int_{\Omega}|u|^{p-2} v w d x .
$$

Por (B.2) e (B.3), temos a existência de $J^{\prime \prime}(u)$,

$$
J^{\prime \prime}(u) v w=p(p-1) \int_{\Omega}|u|^{p-2} v w d x
$$




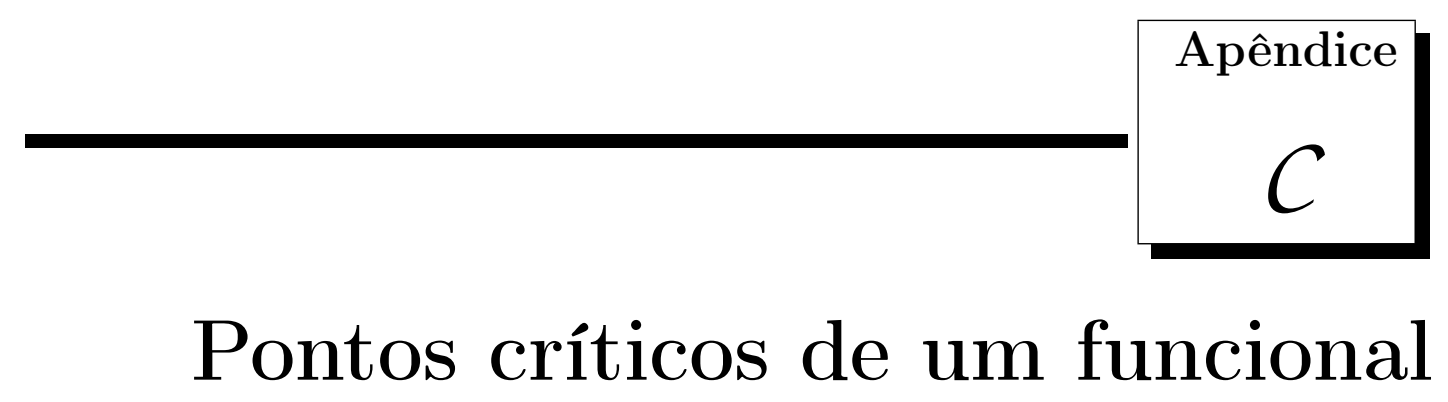

Sejam $X$ um espaço de Banach, $A \subset X$ um conjunto aberto não-vazio e $J: A \longrightarrow \mathbb{R}$ um funcional derivável no sentido Gâteaux em $u \in A$. Dizemos que $u$ é um ponto crítico de $J$ se $J^{\prime}(u)=0$, isto é, se $J^{\prime}(u) v=0$ para todo $v \in X$. É usual chamar a equação $J^{\prime}(u)=0$ de equação de Euler-Lagrange do funcional $J$.

Proposição C.1. Sejam $X$ um espaço de Banach, $A \subset X$ um conjunto aberto não-vazio e $J: A \longrightarrow \mathbb{R}$ um funcional. Se $a \in A$ é minimo local de $J$ e se existir $J^{\prime}(a)$, então a é um ponto crítico de $J$.

Demonstração. Seja $v \in X, v \neq 0$ e defina $f_{v}: \mathbb{R} \longrightarrow \mathbb{R}$ por $f_{v}(t)=J(a+t v)$. Como $a$ é mínimo local de $J$, existe $r>0$, tal que $x$ em $B_{r}(a)$, implica $J(x) \geq J(a)$. Assim para todo $t$ com $|t|<\frac{r}{\|v\|}$ temos

$$
f_{v}(t) \geq f_{v}(0)
$$

Isto é, 0 é um mínimo local de $f_{v}$. Assim, da diferenciabilidade de $J$ e da regra da cadeia, temos que $f_{v}$ é derivável em 0 e

$$
f_{v}^{\prime}(0)=J^{\prime}(a) v
$$

Assim $f_{v}$ é derivável em 0 e possui mínimo local em 0 . Portanto $f_{v}^{\prime}(0)=0$.

Concluímos que $J^{\prime}(a) v=0$ para todo $v \in X$, ou seja, $J^{\prime}(a)=0 \in X^{\prime}$.

Definição C.2. Sejam $X$ um espaço de Banach, $J \in C^{1}(X)$ e c $\in J(X)$ um número real. Dizemos que c é um valor regular para $J$ se para todo $u \in J^{-1}\{c\}$ tem-se $J^{\prime}(u) \neq 0$.

Teorema C.3. Sejam $X$ um espaço de Banach, $K, M \in \mathcal{C}^{1}(X)$, c um valor regular de $K$ e $S=K^{-1}\{c\}$. Se existe $u \in X$ tal que $M(u)=\inf _{v \in S} M(v)$, então existe $\lambda \in \mathbb{R}$, 
chamado de multiplicador de Lagrange, tal que

$$
M^{\prime}(u)=\lambda K^{\prime}(u)
$$

Demonstração. Veja [15, Proposition 14.3].

Teorema C.4. Seja $X$ um espaço reflexivo. Se $J: X \longrightarrow \mathbb{R}$ é semicontínuo inferiormente por sequências na topologia fraca de $X$ e coercivo, então existe um minimo global $u \in X$ para $J$.

Demonstração. Como $J$ é coercivo, existe $R>0$ tal que

$$
J(u) \leq J(0)+1, \forall u \in X \text { tal que }\|u\|>R
$$

Seja $c=\inf _{\bar{B}_{R}} J$, considere $\left(u_{k}\right) \in \bar{B}_{R}(0)$ tal que $J\left(u_{k}\right) \longrightarrow c$.

Como $X$ é reflexivo, a menos de se tomar uma subsequência, existe $u \in \bar{B}_{R}(0)$, tal que $u_{k} \rightarrow u \in \bar{B}_{R}(0)$. Como $J$ é semicontínuo inferiormente por sequências na topologia fraca, temos

$$
c \leq J(u) \leq \liminf _{k \rightarrow \infty} J\left(u_{k}\right)=\lim _{k \rightarrow \infty} J\left(u_{k}\right)=c .
$$

Portanto

$$
J(u)=c=\inf _{\bar{B}_{R}(0)} J(u)=\inf _{X} J(u),
$$

pela escolha de $R$.

Definição C.5. Sejam $J \in \mathcal{C}^{1}(X)$ e $c \in \mathbb{R}$. Dizemos que $J$ satisfaz a condição $(P S)_{c}$ se toda sequência $\left(u_{k}\right) \in X$ tal que

$$
J\left(u_{k}\right) \longrightarrow c, \quad J^{\prime}\left(u_{k}\right) \longrightarrow 0
$$

possui uma subsequência convergente.

Teorema C.6 (Teorema do passo da montanha). Sejam $\mathcal{X}$ um espaço de Banach, $J \in$ $\mathcal{C}^{1}(\mathcal{X}), e \in \mathcal{X}$ e $r>0$ tais que $\|e\|>r$ e ainda

$$
b=\inf _{\|u\|=r} J(u)>J(0) \geq J(e)
$$


Se J satisfaz a condição $(P S)_{c}$ com

$$
c=\inf _{\gamma \in \Gamma}\left(\max _{t \in[0,1]}(J(\gamma(t)))\right)
$$

$e$

$$
\Gamma=\{\gamma \in C([0,1], X) ; \gamma(0)=0, \gamma(1)=e\}
$$

então c é um valor crítico de $J$.

Demonstração. Ver [20, Theorem 2.10]. 


\section{Referências}

[1] R. A. Adams. Sobolev Spaces. Pure and Applied Mathematics, vol. 140. Academic Press, 2003.

[2] V. Adolfsson. L2-integrability of second order derivatives for Poisson's equation in nonsmooth domains. Math. Scand., 1992:146-160.

[3] S. Agmon, A. Douglis, L. Nirenberg. Estimates near the boundary for solutions of elliptic partial differential equations satisfying general boundary conditions. Comm. Pure Appl. Math., n. 12, 1959:623-727.

[4] M. Badiale, F. Serra. Semilinear elliptic equations for beginners: existence results via the variational approach. Universitext - Springer-Verlag. London: Springer, 2011.

[5] T. Boggio. Sulle funzioni di Green d'ordine m. Rend. Circ. Mat., 1905:97-135.

[6] D. Bonheure, E. M. dos Santos, M. Ramos. Ground state and non-ground state solutions of some strongly coupled elliptic systems. Transactions of the American Mathematical Society, vol. 364, n. 1, 2011:447-491.

[7] H. Brezis. Functional analysis, Sobolev spaces and partial differential equations. Universitext. Springer, 2010.

[8] M. P. do Carmo. Differential Geometry of Curves and Surfaces. Englewood, Cliffs, N. Jersey: Prentice Hall, 1976.

[9] A. Dall'Acqua, G. Sweers. The clamped plate equation for the limaçon. Ann. Mat. Pura Appl., n. 184, 2005:361-374.

[10] L. Evans. Partial Differential Equations. Graduate Studies in Mathematics Series. Amer Mathematical Society, 2010.

[11] D. G. de Figueiredo. Equações diferenciais aplicadas. (Coleção matemática universitária). Rio de Janeiro: IMPA, 2002. 
[12] F. Gazzola, H. Grunau, G. Sweers. Polyharmonic Boundary Value Problems: Positivity Preserving and Nonlinear Higher Order Elliptic Equations in Bounded Domains. Lecture Notes in Mathematics. Springer, 2010.

[13] D. A. Gilbarg, N. S. Trudinger. Elliptic Partial Differential Equations of Second Order. Classics in Mathematics Series. Springer-Verlag, 2001.

[14] J. H. Ginsberg. Mechanical and structural vibrations: theory and applications. Wiley, 2001.

[15] O. Kavian. Introduction à la théorie des points critiques et applications aux problèmes elliptiques. Mathématiques \& applications. Paris: Springer-Verlag, 1993.

[16] E. L. Lima. Curso de Análise vol. 2. Projeto Euclides. Rio de Janeiro: IMPA, 2010.

[17] E. Mitidieri. A Rellich type identity and applications. Communications in Partial Differential Equations, vol. 18, n. 1-2, 1993:125-151.

[18] J. Moreau. Décomposition orthogonale d'un espace hilbertien selon deux cônes mutuellement polaires. C. R. Acad. Sci., vol. 255, 1962:238-240.

[19] R. C. A. M. van der Vorst. Variational identities and applications to differential systems. Arch. Rational Mech. Anal., vol. 116, 1991:375-398.

[20] M. Willem. Minimax Theorems. Progress in Nonlinear Differential Equations and Their Applications. Boston: Birkhäuser, 1997. 